\title{
Solar Wind Electrons Alphas and Protons (SWEAP) Investigation: Design of the Solar Wind and Coronal Plasma Instrument Suite for Solar Probe Plus
}

Justin C. Kasper ${ }^{1,2}(\mathbb{D}) \cdot$ Robert Abiad $^{3}$ - Gerry Austin' ${ }^{2}$ Marianne Balat-Pichelin ${ }^{4}$ Stuart D. Bale ${ }^{3} \cdot$ John W. Belcher ${ }^{5}$ Peter Berg ${ }^{3} \cdot$ Henry Bergner $^{2}$. Matthieu Berthomier ${ }^{6}$ - Jay Bookbinder ${ }^{2}$ - Etienne Brodu ${ }^{4}$. David Caldwell ${ }^{2}$. Anthony W. Case ${ }^{2}$ - Benjamin D.G. Chandran ${ }^{7}$ - Peter Cheimets ${ }^{2}$.

Jonathan W. Cirtain ${ }^{8}$ - Steven R. Cranmer ${ }^{9}$ David W. Curtis ${ }^{3}$ - Peter Daigneau ${ }^{2}$. Greg Dalton $^{3}$ - Brahmananda Dasgupta ${ }^{10}$ • David DeTomaso ${ }^{11}$ - Millan Diaz-Aguado ${ }^{3}$. Blagoje Djordjevic $^{3}$ - Bill Donaskowski ${ }^{3}$ Michael Effinger ${ }^{8}$. Vladimir Florinski ${ }^{10}$. Nichola Fox ${ }^{11}$ - Mark Freeman ${ }^{2}$ - Dennis Gallagher ${ }^{8}$ S. Peter Gary ${ }^{12}$. Tom Gauron ${ }^{2}$. Richard Gates $^{2}$ - Melvin Goldstein ${ }^{13}$ - Leon Golub ${ }^{2}$ - Dorothy A. Gordon ${ }^{3}$.

Reid Gurnee $^{11}$ - Giora Guth ${ }^{2} \cdot$ Jasper Halekas $^{14}$ - Ken Hatch ${ }^{3}$ Jacob Heerikuisen ${ }^{10}$. George Ho $^{11}$ • Qiang Hu ${ }^{10}$ - Greg Johnson ${ }^{3}$ Steven P. Jordan ${ }^{2}$ Kelly E. Korreck ${ }^{2}$. Davin Larson ${ }^{3}$ - Alan J. Lazarus ${ }^{5}$ - Gang Li $^{10}$ • Roberto Livi ${ }^{3}$ Michael Ludlam ${ }^{3}$. Milan Maksimovic ${ }^{15}$ - James P. McFadden ${ }^{3}$. William Marchant ${ }^{3}$. Bennet A. Maruca ${ }^{3}$. David J. McComas ${ }^{16,17}$ - Luciana Messina ${ }^{3}$. Tony Mercer ${ }^{3}$. Sang Park ${ }^{2}$ - Andrew M. Peddie ${ }^{13}$ - Nikolai Pogorelov ${ }^{10}$ • Matthew J. Reinhart ${ }^{11}$. John D. Richardson ${ }^{5}$ Miles Robinson ${ }^{3}$ - Irene Rosen ${ }^{3}$ - Ruth M. Skoug ${ }^{12}$. Amanda Slagle $^{3} \cdot$ John T. Steinberg $^{12} \cdot$ Michael L. Stevens ${ }^{2} \cdot$ Adam Szabo $^{13}$. Ellen R. Taylor ${ }^{3}$. Chris Tiu ${ }^{3}$ - Paul Turin ${ }^{3}$ - Marco Velli ${ }^{18}$ - Gary Webb ${ }^{10}$. Phyllis Whittlesey ${ }^{10}$ - Ken Wright ${ }^{10}$. S.T. Wu ${ }^{10}$. Gary Zank ${ }^{10}$

Received: 20 January 2015 / Accepted: 30 September 2015 / Published online: 29 October 2015 (C) The Author(s) 2015. This article is published with open access at Springerlink.com

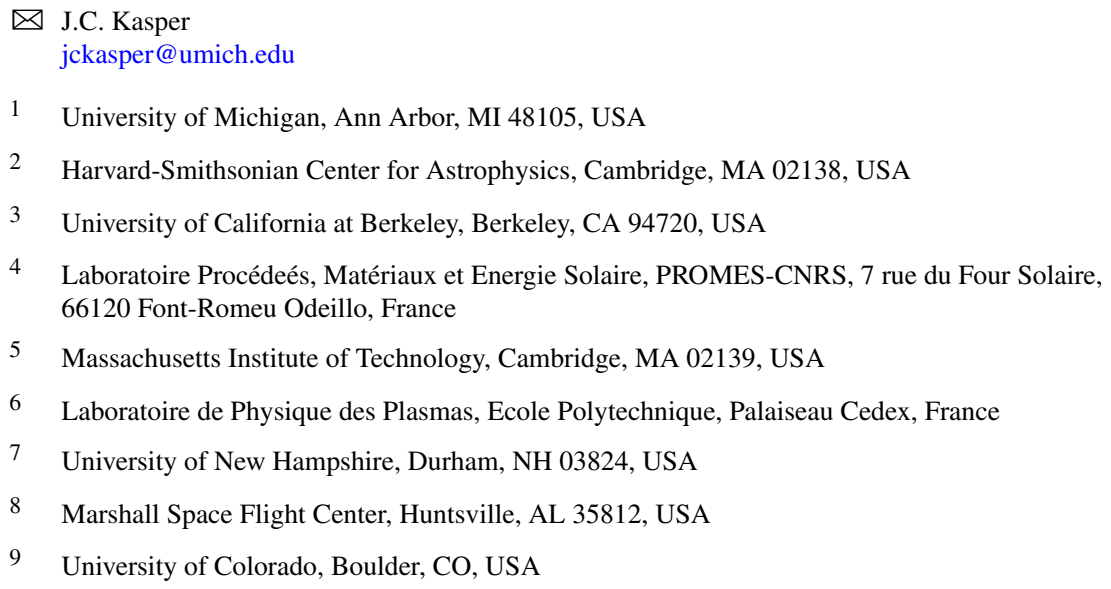




\begin{abstract}
The Solar Wind Electrons Alphas and Protons (SWEAP) Investigation on Solar Probe Plus is a four sensor instrument suite that provides complete measurements of the electrons and ionized helium and hydrogen that constitute the bulk of solar wind and coronal plasma. SWEAP consists of the Solar Probe Cup (SPC) and the Solar Probe Analyzers (SPAN). SPC is a Faraday Cup that looks directly at the Sun and measures ion and electron fluxes and flow angles as a function of energy. SPAN consists of an ion and electron electrostatic analyzer (ESA) on the ram side of SPP (SPAN-A) and an electron ESA on the anti-ram side (SPAN-B). The SPAN-A ion ESA has a time of flight section that enables it to sort particles by their mass/charge ratio, permitting differentiation of ion species. SPAN-A and -B are rotated relative to one another so their broad fields of view combine like the seams on a baseball to view the entire sky except for the region obscured by the heat shield and covered by SPC. Observations by SPC and SPAN produce the combined field of view and measurement capabilities required to fulfill the science objectives of SWEAP and Solar Probe Plus. SWEAP measurements, in concert with magnetic and electric fields, energetic particles, and white light contextual imaging will enable discovery and understanding of solar wind acceleration and formation, coronal and solar wind heating, and particle acceleration in the inner heliosphere of the solar system. SPC and SPAN are managed by the SWEAP Electronics Module (SWEM), which distributes power, formats onboard data products, and serves as a single electrical interface to the spacecraft. SWEAP data products include ion and electron velocity distribution functions with high energy and angular resolution. Full resolution data are stored within the SWEM, enabling high resolution observations of structures such as shocks, reconnection events, and other transient structures to be selected for download after the fact. This paper describes the implementation of the SWEAP Investigation, the driving requirements for the suite, expected performance of the instruments, and planned data products, as of mission preliminary design review.
\end{abstract}

Keywords Solar probe plus $\cdot$ SWEAP $\cdot$ Solar wind plasma $\cdot$ Corona $\cdot$ Heating $\cdot$ Acceleration

\title{
1 Introduction
}

For centuries solar eclipses have provided brief glimpses of the solar corona, a highly structured and magnetized atmosphere that surrounds the Sun and extends throughout the solar system as the supersonic solar wind. Today, more than half a century into the space age, the Sun, corona and interplanetary environment are tracked continuously by observatories

\footnotetext{
10 University of Alabama Huntsville, Huntsville, AL 35805, USA

11 Applied Physics Laboratory, Laurel, MD 20723, USA

12 Los Alamos National Laboratory, Los Alamos, NM 87545, USA

13 Goddard Space Flight Center, Greenbelt, MD 20771, USA

14 University of Iowa, Iowa City, IA 52242, USA

15 Observatoire de Paris, LESIA, UMR CNRS 8109, Meudon 92195, France

16 Southwest Research Institute, San Antonio, TX 78228, USA

17 University of Texas at San Antonio, San Antonio, TX 78249, USA

18

University of California Los Angeles, Los Angeles, CA 90095, USA
} 
on Earth and in space. We know more about solar activity and the impact of space weather on society than ever before, but we have not yet answered fundamental questions about our closest star: Why is the corona millions of degrees hotter than the visible surface of the Sun? How does the corona produce the supersonic and variable solar wind? How are solar flares and coronal eruptions able to produce storms of energetic particle radiation? Successfully answering these questions will represent a major breakthrough in our understanding of the physics of energetic magnetized plasmas, allowing us to better understand and predict the evolution of the corona and solar wind and providing us with general insights into the physics of plasmas from the laboratory to exotic astrophysical environments.

It has long been recognized that the only way to unambiguously answer these questions is to send an instrumented probe close to the Sun to directly sample particles and electromagnetic fields in the corona (e.g., McComas et al. 2007). In 2018 we will finally embark on this journey with the launch of the Solar Probe Plus spacecraft, designed to survive repeatedly plunging through the solar corona to collect the required local measurements. Solar Probe Plus will make use of a series of Venus gravitational assists to gradually move the perihelion of the orbit from 35 solar radii $\left(R_{s}\right)$ down to under $10 R_{s}$ from the center of the Sun within approximately six years (Fox et al. 2015, this issue). The spacecraft is equipped with a diverse payload of scientific instrument suites to investigate the near-Sun environment. The Solar Wind Electrons Alphas and Protons (SWEAP) Investigation, described in this paper, is a four sensor instrument suite that provides complete measurements from several $\mathrm{eV}$ to tens of $\mathrm{keV}$ of the velocity distribution functions of electrons and ionized helium and hydrogen (alpha particles and protons) that constitute the bulk of the solar wind and coronal plasma, along with properties of other ions sorted by their mass/charge. The ISIS suite of energetic particle instruments measure particle fluxes as a function of energy, direction, and composition starting at the upper energies of SWEAP and extending to more than $200 \mathrm{MeV} /$ nucleon for ions and $6 \mathrm{MeV}$ for electrons (McComas et al. 2014). The FIELDS Investigation makes comprehensive measurements of vector electric and magnetic fields from nearly DC values up to frequencies beyond $10 \mathrm{MHz}$ (Bale et al. 2015, this issue). A high-speed interface between SWEAP and FIELDS permits the precise synchronization of plasma and field measurements, along with on-board calculations of wave-particle correlations at high frequency. The WISPR Investigation is a pair of wide field imaging telescopes which will both remotely image the corona and streams of material as the spacecraft passes through them (Vourlidas et al. 2015, this issue).

SWEAP consists of the Solar Probe Cup (SPC), a Sun-viewing fast Faraday Cup designed to operate under extreme temperatures, and the Solar Probe Analyzers (SPAN), a combination of three electrostatic analyzers that make detailed measurements of ion and electron velocity distributions from the shadowed region behind the spacecraft heat shield. SPC precisely measures fluxes and flow angles as a function of energy from $50 \mathrm{eV} / \mathrm{q}$ to $8 \mathrm{keV} / \mathrm{q}$ for ions and $50 \mathrm{eV}$ to $2 \mathrm{keV}$ for electrons. SPAN consists of an ion and electron electrostatic analyzer (ESA) on the ram side of SPP (SPAN-A) and an electron ESA on the anti-ram side (SPAN-B). The SPAN-A ion ESA measures ions as a function of direction and energy/charge from several $\mathrm{eV} / \mathrm{q}$ to $20 \mathrm{keV} / \mathrm{q}$ and has a time of flight section that enables it to sort particles by their mass/charge ratio, permitting differentiation of ion species. The fields of view of SPC and the SPAN-A ion ESA allow SWEAP to continuously track ion flows in the presence of strong waves, nearly subsonic flows, and aberration due to the high orbital speeds of closest approach. The SPAN-A and SPAN-B electron ESAs also run from several $\mathrm{eV}$ to $20 \mathrm{keV}$. SPAN-A and $-\mathrm{B}$ are rotated relative to one another so the broad fields of view of the electron ESAs combine like the seams on a baseball to view the entire sky except for the region obscured by the heat shield and covered by SPC, permitting sensitive 
measurements of electron temperatures, heat fluxes, and field-aligned beams. Observations by SPC and SPAN produce the combined field of view and measurement capabilities required to fulfill the science objectives of SWEAP and Solar Probe Plus. SPC and SPAN are managed by the SWEAP Electronics Module (SWEM), which distributes power, formats onboard data products, and serves as a single electrical interface to the spacecraft. SWEAP data products include ion and electron velocity distribution functions with high energy and angular resolution. Full resolution data are stored within the SWEM, enabling high resolution observations of structures such as shocks, reconnection events, and other transient structures to be selected for download after the fact.

A successful Preliminary Design Review (PDR) for the SWEAP Investigation was held in October 2013. The results of this suite PDR were presented as part of the SPP Mission PDR in January 2014. As the mission has been confirmed, the SWEAP investigation has now entered the detailed design phase. The purpose of this article is to describe the implementation of the SWEAP Investigation at the time of the suite and mission PDRs. This paper describes the scientific goals and driving requirements for the SWEAP Investigation suite, the preliminary designs of all of the instruments, expected instrument performance, planned data products and mission operations, as of the Solar Probe Plus Mission PDR. While it is likely that detailed aspects of the design, operations plan, and data products will evolve before launch, the goal of this work is to provide a representative snapshot of the performance of the suite that may prove useful for researchers interested in the thermal plasma science and measurements possible with SPP.

This paper is organized as follows. The remainder of Sect. 1 describes the scientific basis of the SWEAP investigation and the resulting performance requirements for the SWEAP instruments. Section 2 presents an overview of the organization of the suite. Section 3 covers the Solar Probe Cup sunward facing Faraday Cup. Section 4 describes the SPAN electrostatic analyzers. Section 5 describes SWEAP operations and data products.

\subsection{Science Background and Objectives}

Solar Probe Plus (SPP) provides an unprecedented opportunity to explore the atmosphere of our star, make unexpected discoveries, and enable fundamental understanding of the physics of the corona and solar wind. With an initial perihelion of $35 \mathrm{R}_{\mathrm{s}}$ eventually closing to within $10 \mathrm{R}_{\mathrm{s}}$ from the center of the Sun, SPP will be the first spacecraft to pass from the solar wind into the solar corona. Fueled by convection and magnetic fields, the plasma temperature rapidly rises within hundreds of kilometers of the surface of our Sun from the relatively cool $6000 \mathrm{~K}$ photosphere through a $10000 \mathrm{~K}$ transition region and gives birth to the 1-10 MK solar corona. The hot corona produces a supersonic solar wind that pervades interplanetary space and carves the protective heliospheric bubble out of the interstellar medium. While fifty years have passed since the prediction and detection of the solar wind, we still do not understand how the corona is heated and how the solar wind is accelerated. SPP will directly visit the atmosphere of our star and close the observational gap between remote and in situ observations of the inner heliosphere.

SPP observations will most assuredly challenge our current understanding of the solar wind and corona. Our models for the origin of the wind rest on remote observations and in situ measurements no closer than 0.3 AU from the Sun. In the modern paradigm, the wind is composed of fast and slow streams interspersed with occasional transient coronal mass ejections (CMEs). Properties of slow, fast, and transient wind in the interplanetary medium are summarized in Table 1 . Slow wind generally exhibits highly variable structure and composition but simple ion and electron velocity distribution functions (VDFs) 
Table 1 The standard picture of the in situ solar wind, divided into fast and slow steady state solar wind and transient coronal mass ejections (CMEs)

\begin{tabular}{|c|c|c|c|}
\hline & Slow wind & Fast wind & Transients \\
\hline Likely sources & $\begin{array}{l}\text { Streamer belt, active } \\
\text { regions, coronal hole } \\
\text { boundary layers }\end{array}$ & $\begin{array}{l}\text { Coronal holes, active } \\
\text { regions }\end{array}$ & CMEs \\
\hline Typical speeds & $<400 \mathrm{~km} / \mathrm{s}$ & $700-900 \mathrm{~km} / \mathrm{s}$ & $400-2000 \mathrm{~km} / \mathrm{s}$ \\
\hline Temperatures & $\mathrm{T}_{\mathrm{p}}<\mathrm{Te}$ & $\mathrm{T}_{\mathrm{p}}>\mathrm{Te}$ & Variable \\
\hline $\begin{array}{l}\text { Velocity Distribution } \\
\text { Functions (VDFs) }\end{array}$ & $\begin{array}{l}\text { Nearly Maxwellian, equal } \\
\text { temperatures }\end{array}$ & $\begin{array}{l}\text { Highly nonthermal with } \\
\text { anisotropies, differential } \\
\text { flow, and beams, mass } \\
\text { proportional temperatures }\end{array}$ & $\begin{array}{l}\text { Non-Maxwellian in shocked } \\
\text { plasma, e-beams within } \\
\text { magnetic clouds }\end{array}$ \\
\hline Structure & $\begin{array}{l}\text { Filamentary, highly } \\
\text { variable }\end{array}$ & Uniform, slow changes & Shocks, magnetic clouds \\
\hline $\begin{array}{l}\text { Elemental } \\
\text { abundance }\end{array}$ & Distinct fractionation & Coronal & Highly variable \\
\hline Composition & $\mathrm{He} / \mathrm{H} \sim 1-5 \%$, variable & $\mathrm{He} / \mathrm{H} \sim 5 \%$, steady & $1-30 \%$ \\
\hline
\end{tabular}
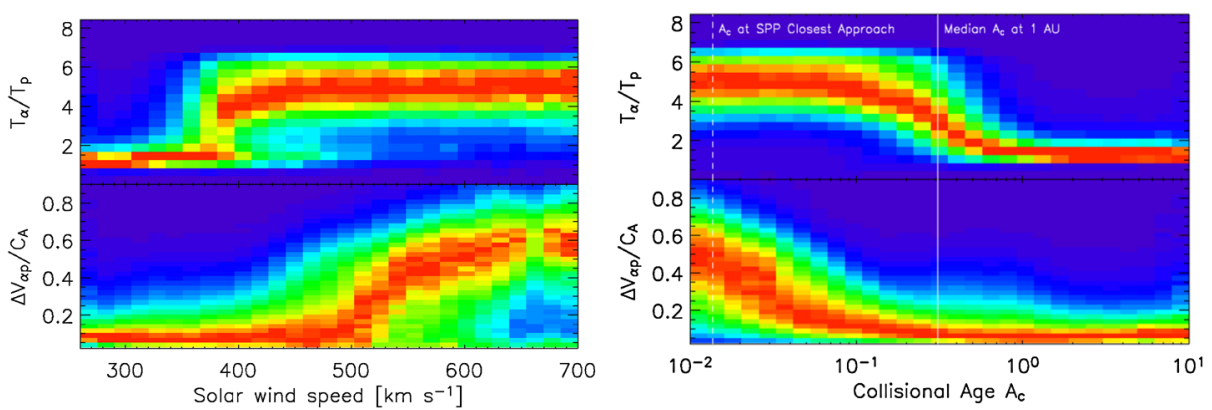

Fig. 1 Distribution of the temperature ratio $\left(\mathrm{T}_{\alpha} / \mathrm{T}_{\mathrm{p}}\right)$ of alphas to protons and the differential flow between the species normalized by the Alfvén speed $\left(\Delta \mathrm{V}_{\alpha \mathrm{p}} / \mathrm{C}_{\mathrm{A}}\right)$ as function of solar wind speed (left) and collisional age $\mathrm{A}_{\mathrm{c}}$ (right) using the same set of four million Wind/FC observations. A smaller number of collisions during propagation to Earth seems to be more important than speed in determining if the plasma will be non-Maxwellian. Vertical lines indicate the median value of $\mathrm{A}_{\mathrm{c}}$ at $1 \mathrm{AU}$ (solid) and at SPP closest approach (dashed), suggesting SPP may discover that all wind is non-Maxwellian near the Sun

that are close to Maxwellian and have similar temperatures. Fast wind is less variable, but often has strong fluctuations and non-Maxwellian VDFs, including different temperatures, anisotropies, and velocities between ions. These non-Maxwellian properties are believed to be signatures of the wave-particle coupling responsible for the high speed and mass flux of the fast wind. By observing below 10 solar radii $\left(R_{s}\right)$ of the Sun SPP will directly probe the solar wind as it emerges from the corona and establish direct connections between the wind and source regions on the Sun. SPP is also very likely to produce surprises. A simple analysis of solar wind at 1 AU highlights how our current paradigm (Table 1) is distorted by our biased view from beyond 0.3 AU (Kasper et al. 2008; Maruca et al. 2013). Figure 1 shows the distribution of the temperature ratio and differential flow between alphas and protons as a function of speed (left panel) and collisional age $\mathrm{A}_{\mathrm{c}}$ (the Coulomb collision frequency multiplied by the transit time of the wind from the Sun to the Wind spacecraft at $1 \mathrm{AU})$. We see that low $\mathrm{A}_{\mathrm{c}}$ is a much better predictor of non-Maxwellian features than solar wind speed. Slow wind, with a longer transit time and 


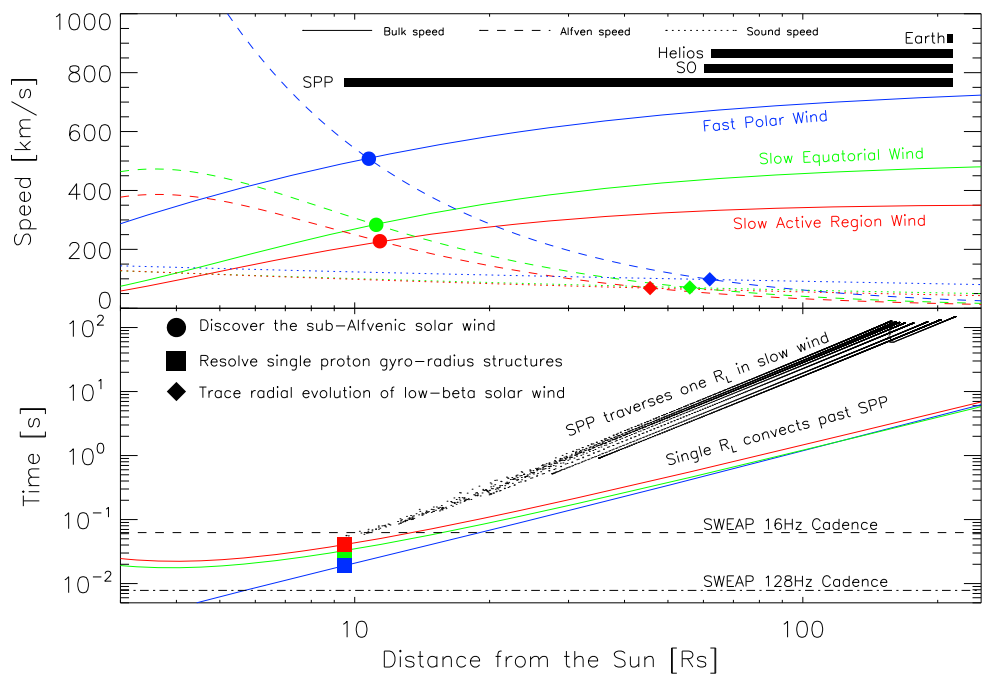

Fig. 2 Top: bulk speed, Alfvén speed, and sound speed for models of fast polar, slow equatorial, and slow active region solar wind (Cranmer et al. 2007) along with the orbits of various objects. SPP will provide unique observations of low-beta plasma (distances below the diamonds) and sub-Alfvénic plasma (circles). Bottom: time for one proton gyro-radius $\left(\mathrm{R}_{\mathrm{L}}\right)$ to radially drift past SPP (colored lines) and for SPP to traverse one $\mathrm{R}_{\mathrm{L}}$ due to orbital motion above the surface of the Sun (dots). Horizontal lines indicate SWEAP cadence for bulk measurements $(16 \mathrm{~Hz})$ and for burst flow angle and flux measurements $(128 \mathrm{~Hz})$, showing that SWEAP can resolve single $\mathrm{R}_{\mathrm{L}}$, even at closest approach (squares)

higher collision rate washes out these signatures of wave-particle processes, suggesting rich physics will be revealed by SPP upon close approach. This hints at the surprises that we may anticipate with SPP and suggests that we should not take our current understanding of the basic solar wind, its origin and properties, as established.

The remainder of this section describes the SWEAP science goals and measurement requirements, as developed based on an extensive analysis of existing models and observations. Figure 2 summarizes some characteristic speeds and temporal scales that SPP will encounter as a function of distance from the Sun for reference, based on a simple extrapolation of solar wind plasma observations from the Helios spacecraft combined with the baseline orbital trajectory and velocity of the SPP spacecraft. Simulations of the radial dependence of fast solar wind from a coronal hole and slow wind from active regions and the streamer belt were compared with extrapolations of Helios observations from 0.3-1 AU and remote coronal diagnostics for consistency and a best guess of solar wind conditions was produced. These predictions guide the science goals and measurement requirements established below. Where possible we have designed SWEAP to detect not only the average expected range of critical parameters but also as much of the extremes as possible.

SWEAP science objectives are organized under three overarching scientific objectives which also match the overall objectives of the mission. These three objectives are:

1. Sources of the solar wind: Determine the structure and dynamics of the magnetic fields at the sources of the fast and slow solar wind.

2. Heating the corona and solar wind: Trace the flow of energy that heats the solar corona and accelerates the solar wind.

3. Acceleration and transport of energetic particles: Explore mechanisms that accelerate and transport energetic particles. 
The following subsections describe these three overarching objectives for SWEAP by expending each objective into a series of distinct goals. For each goal, we review current observations, open questions, proposed theories, and the observations SWEAP instruments need to perform in order to achieve significant scientific closure. Generally this involves specifying the resolution in time, energy, angle, or particle type needed to distinguish between classes of competing physical theories. With these measurement requirements established, we can develop the resulting performance requirements for SWEAP and the individual sensors within the suite. Before proceeding, we note two important caveats. First, the following discussion of scientific objects and required data products and measurement requirements is focused on SWEAP instruments. This focus is done simply to allow us to concentrate on the performance requirements for SWEAP. The overall review of the scientific objectives of SPP published in this volume describes how the combined capabilities of all the instrument suites further strengthens the scientific capabilities of this mission (Fox et al. 2015, this issue). Second, we note that our aim in this section is not to present a comprehensive review of all current outstanding questions and candidate theories, but instead to identify the range of observational needs that set the extremes of SWEAP instrument performance requirements.

\subsubsection{Sources of the Solar Wind}

The first scientific objective for SWEAP is to determine the structure and dynamics of the magnetic fields at the sources of the fast and slow solar wind. SWEAP provides the data products throughout each solar encounter required to identify the location and physics of solar wind sources. Robotic exploration of the heliosphere has produced fifty years of in situ solar wind measurements. These data, combined with remote solar and coronal imaging and spectroscopy, shape our understanding of the global magnetic and plasma connections from the surface of the Sun through interplanetary space. By associating solar wind speeds, composition, temperature, and non-Maxwellian properties with coronal features we discover the source regions of the solar wind: fast wind associated with coronal holes, slow wind emerging either from the streamer belt or active regions, and coronal mass ejections (CMEs) producing transient solar wind at all speeds. SPP and SWEAP will allow us to move from discovery of solar wind sources to understanding the underlying physics: How do these source regions map into the heliosphere and what fraction of the corona is actually magnetically open to the heliosphere at any instant in time and over the solar cycle? If slow wind emerges from the streamer belt, how does it extend to such a large range in latitude? What fraction of small-scale structures in the interplanetary medium, from magnetic holes and reconnection exhausts to density fluctuations and magnetic discontinuities are signatures of coronal physics or features that develop within the solar wind during propagation? We address these questions through the following four goals: (1) Connect the large-scale structure of the solar wind to solar sources, (2) Understand how the solar wind is accelerated, (3) Understand the variable connection between the corona and the solar wind, and (4) Discover the smallest coronal structures embedded in the solar wind.

(1) Connect the Large Scale Structure of the Solar Wind to Solar Sources Fundamental questions remain about solar wind source regions, especially for the slow wind. What fraction of the slow solar wind, if any, emerges from the streamer belt, from active regions, and from the boundaries of the coronal holes? Another fifty years of observations from our existing vantage points in the heliosphere will not resolve these questions because of fundamental limits in our ability to map solar wind conditions back to their solar surfaces due to 
interactions between faster and slower parcels of plasma and Coulomb relaxation (Fig. 1). SPP provides a platform for measurements of the wind originating from the corona with minimal propagation effects, as larger scale stream interactions develop above heights of $0.25 \mathrm{AU}$ and the plasma will be collisionally young. To conduct these mappings, SWEAP must observe the bulk properties of the solar wind from below $10 \mathrm{R}_{\mathrm{s}}$ out to $0.25 \mathrm{AU}$ with time resolution sufficient to resolve structures the size of a single proton gyro-radius. These science observations necessary to address the science questions posed lead to the following measurement requirements: Observe alpha particle and proton velocity, density, and temperature $\left(\mathbf{V}_{\alpha}, \mathrm{n}_{\alpha}, \mathrm{T}_{\alpha}, \mathbf{V}_{\mathrm{p}}, \mathrm{n}_{\mathrm{p}}, \mathrm{T}_{\mathrm{p}}\right)$ at $1 \mathrm{~Hz}$ and at least $90 \%$ of the time within $0.25 \mathrm{AU}$. Observe e-density and temperature $\left(\mathrm{n}_{\mathrm{e}}, \mathrm{T}_{\mathrm{e}}\right)$ at $1 \mathrm{~Hz}$ at least $90 \%$ of the time within $0.25 \mathrm{AU}$.

(2) Understand How Slow Solar Wind Is Accelerated There are many competing theories for the sources of the slow solar wind. Some models attribute the final solar wind speed to differences in the expansion of the magnetic flux and would produce sharp drops in speed near the heliospheric current sheet (HCS) (Wang 1993). In other models solar wind is inherently fast, but the Kelvin-Helmoltz instability produces a band of slow wind in the vicinity of the HCS that would be correlated with strong velocity shear (Einaudi et al. 1999). Observations made by the LASCO and later SECCHI coronagraphs suggest that the slow wind may indeed be produced in a non-steady fashion. Plasmoids are seen to disconnect from the tips of helmet streamers and propagate away from the Sun (Sheeley et al. 1997). Flattened remnants of these plasmoids can be seen at great distances by the HI instruments on STEREO (Sheeley et al. 2009). These plasmoids are produced by magnetic reconnection, either near the base of the HCS where it extends from the streamer or at the tip of the streamer itself. Measurements of density enhancements of several percent correlated with rapid changes in the orientation of the local magnetic field, along with sudden changes in the relative abundance of helium could be used to inventory the occurrence rate of the plasmoids (Viall et al. 2009). Variations in helium abundance can also be related to solar wind speed, distance from the heliospheric current sheet, and coronal temperature (Kasper et al. 2012; Schwadron et al. 2011, 2014). Another model with ejection of material through reconnection begins deeper in the corona where the closed fields of streamers and the open fields of coronal holes are separated by a boundary. At the boundary, these fields will generally be misaligned, implying a current sheet and the possibility of magnetic reconnection. If reconnection occurs, high pressure streamer plasma is released into the open field line, where it can then escape into the solar wind and be detected through measurements of plasma and field pressure (Wang and Sheeley 2004). To answer these questions we require observations of solar wind bulk parameters across at least $10 \mathrm{HCS}$ crossings within $20 \mathrm{R}_{\mathrm{s}}$ and at least $40 \mathrm{HCS}$ crossings within $0.25 \mathrm{AU}$ (at $68 \%$ confidence level) to determine with significance the signatures of slow solar wind near the streamer belt; Bulk proton parameters at $16 \mathrm{~Hz}$ to detect variation within a single gyro-radius; $\mathrm{n}_{\alpha} / \mathrm{n}_{\mathrm{p}}$ at $1 \mathrm{~Hz} ; \mathrm{T}_{\mathrm{e}}, \mathrm{T}_{\alpha}$, and $\mathrm{T}_{\mathrm{p}}$ and temperature anisotropy to measure total internal pressure.

(3) Understand the Variable Magnetic Connection Between the Corona and the Solar Wind Existing observations are not sufficient to determine the fraction of the corona magnetically open to interplanetary space, the change in the total open flux over the solar cycle, and the variability of the magnetic connectivity on short timescales. Measuring this variable connection is essential to understanding how the solar corona changes over the solar cycle, how open flux is transferred into interplanetary space, and possibly how the corona is heated and the solar wind accelerated. These measurements would permit tests of the importance of interchange reconnection, a model that aims to jointly explain coronal 
Fig. 3 Suprathermal electrons are valuable probes of the magnetic connectivity from SPP to the Sun because the corona must constantly replenish the strahl. This figure is an example of the pitch angle distribution for $272 \mathrm{eV}$ electrons observed by ACE SWEPAM over one day illustrating BDEs and dropouts consistent with the spacecraft passing through closed and open field lines

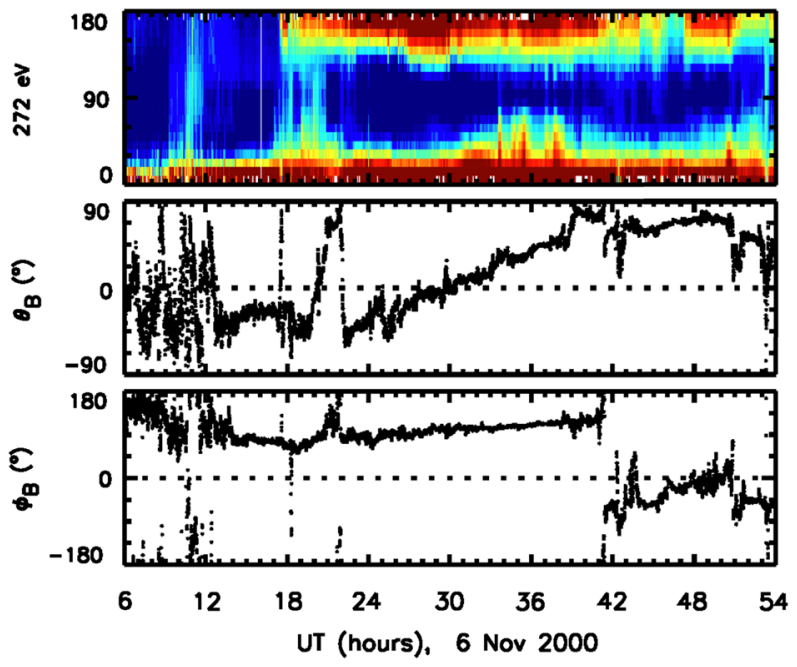

heating and solar wind formation by proposing that open magnetic field lines are present all over the Sun, including regions that are predominantly closed (Fisk and Schwadron 2001; Crooker et al. 2002). Reconnection between an open field line and one end of a closed magnetic loop effectively causes the footpoint of the open line to jump discontinuously to the other end of the loop. At the same time, high pressure plasma is released into the open field line and escapes. The pitch angle distribution (PAD) of suprathermal electrons and the field-aligned e-strahl are valuable probes of the magnetic field topology between solar wind sources and SPP. Suprathermal electrons with energies of hundreds of eV to several $\mathrm{kV}$ are highly mobile, and freely escape along field lines into the heliosphere. Bidirectional electrons (BDEs or counterstreaming electrons) indicate closed field line (loop) structures reaching from the corona into the solar wind (Gosling et al. 1987). Figure 3 provides an example of the appearance of BDEs over a 54 hour period as the ACE spacecraft passed through a magnetic cloud where both ends of the field lines connected back to the corona. Electron PADs can also be used to identify partial field line disconnection from Sun, due to interchange reconnection and motion of field line footpoints on the solar surface. If the measurement cadences are higher than the time to cross a proton gyro-radius SWEAP can repeatedly sample solar wind conditions within a single flux tube to identify the variability in connectivity along a single flux tube. Understanding the variability of the magnetic connection between SPP and the corona requires electron pitch angle distribution with angular resolution $<10^{\circ}$ and burst observations of the total electron strahl flux at up to $100 \mathrm{~Hz}$.

(4) Discover the Smallest Coronal Structures Embedded in the Solar Wind In situ observations of the solar wind show that it is a complex mix of structures on small scales, from current sheets and discontinuities to density fluctuations and magnetic holes. An open question is to determine the fraction of these structures that have developed in the solar wind during propagation or are relics of coronal phenomena. Borovsky (2008) and Li (2008) have shown that magnetic discontinuities in the solar wind may be fossil structures originating from the solar surface related to granule and supergranule structure on the Sun. Plasma structures and boundaries over a range of radial distances allow us to directly address the evolution of structures from the corona into the solar wind. These include the width of the streamer belt, flux tubes, discontinuities, shocks, current sheets, fast/slow wind transitions, 
Fig. 4 An x-ray image of a narrow polar plume is suggestive of the fine scale structures SPP may discover at perihelion

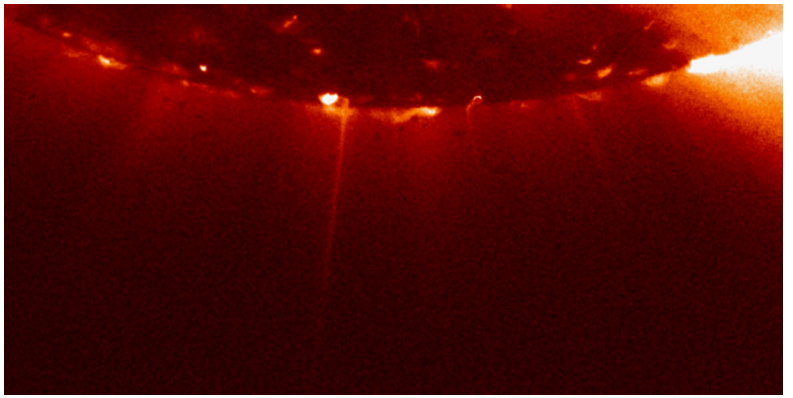

flux ropes/CMEs (plasmoids), and filaments/plumes/jets. Fine-scale structures on scales of $1000 \mathrm{~km}$ or less such as coronal hole plumes extend from the coronal base into the low corona and may survive up to an altitude of $10 \mathrm{R}_{\mathrm{s}}$. Recent observations from Hinode reveal that jets of various sizes occur in the solar atmosphere with a much greater frequency than previously thought. These include X-ray coronal jets (Cirtain et al. 2007) and a newly discovered class of fast $50-150 \mathrm{~km} \mathrm{~s}^{-1}$ spicules (de Pontieu et al. 2007, 2009). Figure 4 is an example of one such highly collimated structure that may survive to SPP distances. These spicules are very thin $(200 \mathrm{~km})$ and occur at a great frequency over the entire solar surface. Whether they will be detectable as individual events by SPP is an open question. Coronal $\mathrm{X}$-ray jets, on the other hand, are sufficiently isolated that their solar wind signature should be clearly measurable. Polar plumes are observationally linked to jets (Raouafi et al. 2008) and are visible in coronagraph images out to at least $30 \mathrm{R}_{\mathrm{s}}$ (De Forest et al. 2001). Variation in e-strahl and PAD correlated with Type-III radio emission (prompt radio emission from beams of electrons escaping from the corona) will determine if SPP is sampling the open flux tube that contains the jet material. In those cases we can determine whether the jet is an important source of the solar wind mass flux within the tube. Bulk plasma properties produced by SWEAP at $16 \mathrm{~Hz}$ are sufficient to resolve these structures of scale sizes of many $\mathrm{R}_{\mathrm{L}}$ (one $\mathrm{R}_{\mathrm{L}}$ at $10 \mathrm{R}_{\mathrm{s}}$ is a few $\mathrm{km}$ ). Electron strahl and pitch angle distributions at $1 \mathrm{~Hz}$ are sufficient to identify sudden changes in magnetic connectivity.

The SWEAP solar wind observations described above, when combined with measurements from other instruments on the spacecraft, remote measurements of coronal and photospheric structure, and theoretical models of the corona, will transform our understanding of the structure and dynamics of the sources of the solar wind.

\subsubsection{Heating the Corona and Solar Wind}

The second scientific objective for SWEAP is to trace the flow of energy that heats the solar corona and accelerates the solar wind. SWEAP measurements are designed to allow us to understand how the solar corona and wind are heated. Arguably the most significant open question in heliophysics is the identification of the physical process or processes responsible for the high temperature of the solar corona and the continued heating of the solar wind in interplanetary space. Given the loss of energy in the corona through radiation, heat conduction, waves, and the escaping solar wind a significant amount of power is required to maintain the observed coronal temperatures. The ultimate source of this energy is the convective motion of the surface of the photosphere and its embedded magnetic field, but the mechanism by which the large scale and low frequency motion of the field is able to dissipate sufficient heat in the corona has not been identified. Spectroscopic measurements of the corona show that heavy ions have large perpendicular temperature anisotropies, with $\mathrm{T}_{\perp} / \mathrm{T}_{\|}>10$. In situ 
Fig. 5 This plot shows the relative density of the electron core, halo, and strahl as a function of distance from the Sun as observed by Helios (adapted from Štverák et al. 2009). In the inner heliosphere the e-strahl will dominate the heat flux

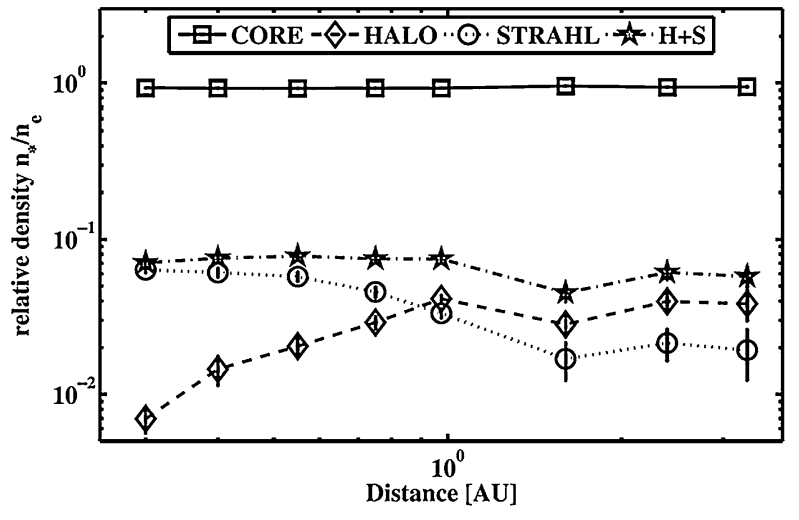

measurements have confirmed that heating also continues in the solar wind out to at least 1 AU. The temperatures of ions and electrons fall more slowly with distance than adiabatic expansion would predict. In the solar wind the proton magnetic moment is not conserved and in fact increases with distance from the Sun, also suggesting preferential heating. In the solar wind with few Coulomb collisions, alpha particles and heavier ions have temperatures proportional to mass, and differential flow relative to the protons at up to the local Alfvén speed. In both cases evidence suggests that the heating is likely due to the breakdown of the plasma as a simple fluid, and the dissipation of fluctuations through a wave-particle coupling process. Accurate and fast solar wind measurements are required to address these processes.

SPP observations will shed valuable light on the dominant heating mechanisms in the corona because the solar wind within $0.25 \mathrm{AU}$ is much more similar to coronal plasma in terms of temperature, plasma beta, and the intensity of fluctuations. The science to be conducted takes three forms: (1) establish the energy budget of the solar wind as a function of distance from the Sun, (2) examine the details of the solar wind for the signatures of the known theoretical heating processes, and (3) identify the role of other limits to ion VDFs, such as plasma micro-instabilities and Coulomb relaxation.

(1) Measure the Energy Budget of the Solar Wind The energy contained in the solar wind as a function of distance from the Sun consists of the bulk kinetic energy of the ions and electrons, the internal energy stored in differential flow, heat fluxes, and different species temperatures and anisotropies, and energy contained within plasma fluctuations. It is well know that most of the heat flux in the solar wind is carried by the e-strahl population (Pilipp et al. 1987). The observation of the radial evolution of this population by SPP is thus a key element in understanding the dynamics of the wind. An analysis of the e-core, halo and strahl measured by HELIOS ( 0.3 to $1 \mathrm{AU}$ ) suggests that within $0.25 \mathrm{AU}$ the e-VDF will likely consist of only a core population plus a narrow strahl centered around 2-3 times the core thermal speed and carrying about $8-10 \%$ of the total e-density (Fig. 5). Tracking the energy of the solar wind ions requires velocities with $1 \%$ uncertainty, densities with better than $5 \%$ uncertainty, and temperatures with less than $20 \%$ uncertainty and occasional measurements of the proton flow angle above $30 \mathrm{~Hz}$ to measure wave power at breakpoint in dissipation. A similar requirement is placed on the accuracy of electron temperature measurements, and to track the plasma heat flux we also must be able to measure within $5^{\circ}$ of the magnetic field to determine the electron strahl whenever possible within $0.25 \mathrm{AU}$.

(2) Understand Which Heating Mechanisms Dominate as a Function of Distance from

the Sun Numerous models to describe the heating and acceleration mechanisms of the 
Table 2 The five categories of models of solar wind heating have distinguishing signatures that SWEAP is designed to detect unambiguously

\begin{tabular}{|c|c|c|c|c|c|}
\hline Mechanism & Ion cyclotron $^{\mathrm{a}}$ & Turbulent cascade ${ }^{\mathrm{b}}$ & $\begin{array}{l}\text { Shock steepened } \\
\text { acoustic modes }\end{array}$ & $\begin{array}{l}\text { Reconnection } \\
\text { and } \\
\text { nano-flares }^{\mathrm{d}}\end{array}$ & Filtration $^{\mathrm{e}}$ \\
\hline Process & $\begin{array}{l}\text { Ion-cyclotron } \\
\text { wave damping }\end{array}$ & $\begin{array}{l}\text { Turbulent cascade, } \\
\text { spectral coupling, } \\
\text { and dissipation }\end{array}$ & $\begin{array}{l}\text { Compressive } \\
\text { waves, wave } \\
\text { steepening, } \\
\text { shock } \\
\text { dissipation }\end{array}$ & $\begin{array}{l}\text { Large and } \\
\text { small-scale } \\
\text { reconnection, } \\
\text { convective } \\
\text { motions }\end{array}$ & $\begin{array}{l}\text { Kappa } \\
\text { distributions }\end{array}$ \\
\hline $\begin{array}{l}\text { Signatures in } \\
\text { the solar wind }\end{array}$ & $\begin{array}{l}\text { high frequency } \\
\text { fluctuations at } \\
\text { ion-cyclotron } \\
\text { frequency, i+ } \\
\text { anisotropy } \\
\mathrm{T}_{\perp}>\mathrm{T}_{\|}\end{array}$ & $\begin{array}{l}\text { low-frequency } \\
\text { MHD and kinetic } \\
\text { Alfven waves, } \\
\text { spectral breaks, } \\
\text { dissipation range }\end{array}$ & $\begin{array}{l}\text { Ion beams, } \\
\text { compressible } \\
\text { low-frequency } \\
\text { waves and } \\
\text { shocks }\end{array}$ & $\begin{array}{l}\text { High speed i+ } \\
\text { beams and } \\
\text { bidirectional } \\
\text { plasma jets, } \\
\text { enhanced } \\
\text { e-strahl }\end{array}$ & $\begin{array}{l}\text { Ions and } \\
\text { electrons } \\
\text { have unusual } \\
\text { peaked VDFs }\end{array}$ \\
\hline $\begin{array}{l}\text { Measurement } \\
\text { requirement }\end{array}$ & \multicolumn{2}{|c|}{$\begin{array}{l}\mathrm{p}+\text { flow angles, } 2 \mathrm{D} \mathrm{i}+\text { and } \mathrm{e}-\mathrm{VDF} \text { at } \\
64 \mathrm{~Hz} \text { cross-correlated with } \mathrm{E} \text { and } \mathrm{B} \\
\text { power spectra }\end{array}$} & $\begin{array}{l}\text { Density and } \\
\text { velocity at } 1 \% \\
\text { accuracy to } \\
\text { detect weak } \\
\text { shocks }\end{array}$ & $\begin{array}{l}\text { Flow angles } \\
\text { and } \mathrm{i}+\text { beams } \\
\text { at } 30 \mathrm{~Hz}\end{array}$ & $\begin{array}{l}\mathrm{dE} / \mathrm{E}<5 \% \\
\text { to resolve } \\
\text { peaked VDF, } \\
\text { p+ and } \\
\text { e-halo }\end{array}$ \\
\hline
\end{tabular}

${ }^{\text {a} H o l l w e g ~(2008), ~ C r a n m e r ~(2000), ~ H o l l w e g ~ a n d ~ I s e n b e r g ~(2002), ~ I s e n b e r g ~(2001 a), ~ G a l i n s k y ~ a n d ~ S h e v c h e n k o ~}$ (2000), Marsch and Tu (2001), Kasper et al. (2008, 2013)

${ }^{b}$ Matthaeus et al. (1999), Dmitruk et al. (2002), Cranmer et al. (2007), Chandran et al. (2009)

${ }^{\mathrm{c}}$ Bruner (1978), Ulmschneider (1985)

${ }^{d}$ Parker (1979, 1987), Sturrock (1999), Priest et al. (2002), Axford and McKenzie (1992), Cargill and Klimchuk (2004), Schrijver et al. (1997), Zurbuchen et al. (2002)

e Scudder (1994), Pierrard and Lamy (2003)

solar corona and wind have been advanced with varying degrees of success, but none is universally accepted and possibly many are valid in some subset of plasma conditions. With the correct measurements SPP can determine the relative contribution of these mechanisms in the solar wind and understand which are most important. It is quite likely that we will discover that some mechanisms are more appropriate to different solar wind speeds, distances from the Sun, and level of solar activity. We have surveyed the range of candidate models and divided them into the five categories shown in Table 2 based on the energy dissipating process. For each category of model the distinguishing signatures that would be found in the solar wind were identified. The final row of the table shows the one aspect of measuring that model that drives instrument performance.

Ion Cyclotron Heating Along with turbulence ion-cyclotron resonant heating is one of the most widely accepted mechanisms for coronal heating. The conversion of magnetic energy due to photospheric-driven motion of the magnetic carpet or even the higher canopy of magnetic field via either direct heating (some form of reconnection) or the launching of waves (Axford and McKenzie 1992; Schrijver et al. 1997; Longcope et al. 2003) is generally thought to be the origin of the energy needed to heat the corona. Ion cyclotron heating and the turbulent cascade models exploit the notion that low frequency shear Alfven waves excited in the chromosphere will survive into the lower corona. Upward propagating Alfven modes have been observed in the chromosphere (de Pontieu et al. 2007), in the lower corona 
by Hinode (Ofman and Wang 2007; Okamoto et al. 2007) and by ground-based detectors (Tomczyk et al. 2007). The ion cyclotron heating mechanism proceeds by resonant wave particle interactions with Alfven waves, leading to heating of the solar corona (Isenberg 2001b, 2004). Recently Wind FC measurements have been used to statistically provide the first in situ evidence for ion heating via an Alfven-ion cyclotron resonant absorption process (Kasper et al. 2008, 2013). Distinctive signatures are fluctuations associated with ion cyclotron and shear Alfven modes in the inertial range and highly anisotropic VDFs. For distances greater than $20 \mathrm{R}_{\mathrm{s}} \mathrm{n}_{\mathrm{p}}, \mathbf{V}_{\mathrm{p}}$, and $\mathrm{T}_{\mathrm{p}}$ at $16 \mathrm{~Hz}$ is sufficient to measure ion fluctuations in the inertial range. In order to measure power in ion fluctuations in the inertial range close to the Sun it is sufficient to measure p-flow angles and total flux faster than $50 \mathrm{~Hz}$. 2D p and $\alpha$ VDFs at $1 \mathrm{~Hz}$.

The Turbulent Cascade An important alternative to ion cyclotron heating is the possibility that upwardly propagating low-frequency Alfven waves are partially reflected, thereby driving a turbulent cascade through coupling to zero frequency modes (Matthaeus et al. 1999; Chandran et al. 2009). The cascade is expected to be quasi-perpendicular and could potentially lead to quasi-perpendicular heating. Velocity and density fluctuation data at the convected proton gyro-frequency (up to tens of $\mathrm{Hz}$ at $9.5 \mathrm{R}_{\mathrm{s}}$ ) is needed to assess the importance of the turbulence cascade. Furthermore, although the kinetic-turbulence picture at dissipation scales remains to be elucidated, intriguing high-frequency/small k observations (Leamon et al. 1998) suggest that the nearly incompressible picture of MHD in a low plasma beta environment with predominantly $2 \mathrm{D}$ behavior may shape the plasma distribution. $2 \mathrm{D}$ velocity distribution data that will shed light on the turbulence cascade and possibly distinguish it from the ion cyclotron model. The heating of the plasma will be orthogonal to $B$, so anisotropic proton VDFs might be expected. Requirements: 2D VDF at $30 \mathrm{~Hz}$ to identify relative resonance with inwards and outward propagating waves at local cyclotron frequency.

Reconnection and Nanoflares Historically, explanations for the heating of the corona, regardless of whether the wind is slow or fast, have focused on the formation of current sheets and reconnection at various scales at the base of the corona (Parker 1988); dissipation then occurs via nanoflares or microflares. Magnetic field and plasma fluctuations have not been measured on these scales and the original mechanism has been the source of much debate. The extrapolation of small scale flaring events to SPP through fine-scale plasma fluctuations and beams is not obvious but one might expect specific power law distributions (Georgoulis et al. 1998) in velocity to result. Other signatures of small-scale reconnection might include bi-directional beams or jets of electrons and ions, energized particles, and even velocity gradient correlations. Requirements: $20 \%$ energy resolution to detect $\mathrm{p}$ - and e-beams in VDFs.

Shock Steepening Coronal heating through shock-steepened acoustic modes is no longer a popular model, although it has been suggested that this may be important in heating the chromosphere. However, if magnetoacoustic modes are present in the corona they could lead to significant heating (Ulmschneider and Stein 1982; Hollweg 1982), and this may be important for the slow solar wind. Requirements: $n_{p}$ fluctuations at $16 \mathrm{~Hz}$ to identify weak or remnant coronal shocks and to resolve their structures (thickness of the order of $\mathrm{R}_{\mathrm{L}}$ ).

Filtration In these models particles from the non-thermal wings of the coronal proton distribution (created in the coronal region intermediate to the collisional and collisionless 

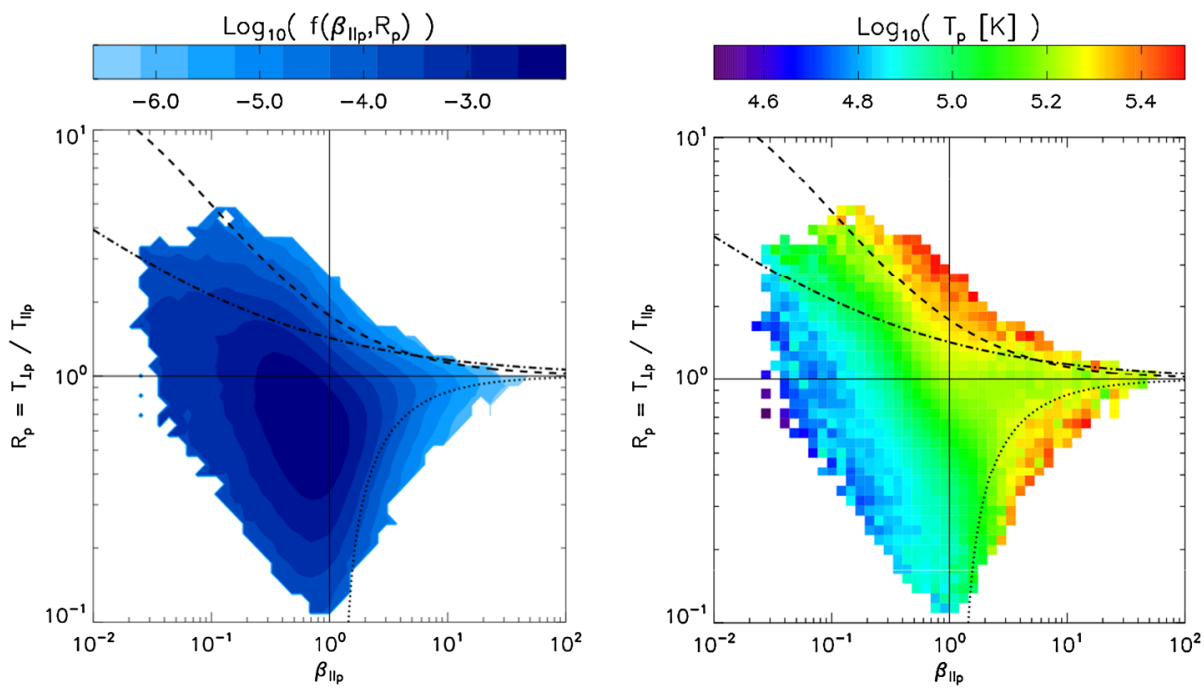

Fig. 6 Left: Distribution of solar wind observations at $1 \mathrm{AU}$ as a function of proton temperature anisotropy and plasma beta. Curves indicate limits to anisotropy imposed by the firehose (dotted), mirror (dashed), and cyclotron (dot-dashed) instabilities. Right: Mean proton temperature as a function of location on these plots, suggesting that heating mechanisms push plasma towards the instability thresholds. Will all the plasma near the Sun be pinned against the instabilities in the upper left corner of these plots?

regimes) escape outward preferentially as a result of velocity filtration by the Sun's gravitational potential. The preferential escape of the high energy tails produces distinctive peaked $\mathrm{i}+$ and e-VDFs that do not survive to previously explored distances due to instabilities and Coulomb collisions. Requirements: 2D p and e-VDFs out to $20 \mathrm{keV}$ with $10 \%$ energy resolution.

(3) Understand the Limits Imposed by Instabilities and Coulomb Relaxation SPP is an opportunity to understand not only the heating mechanisms that distort i+ and e-VDFs near the Sun but also the plasma micro-instabilities that can grow in non-Maxwellian distributions and limit the distortion of the VDF. Figure 6 illustrates the value of SPP measurements to addressing instabilities. Both plots were generated using several million Wind FC measurements of the solar wind. The panel on the left shows the observed distribution at $1 \mathrm{AU}$ of three million measurements as a function of proton temperature anisotropy $\mathrm{R}_{\mathrm{p}}$ and proton parallel plasma beta $\beta_{\| \mathrm{p}}$. The range in $\mathrm{R}_{\mathrm{p}}$ accessible to the solar wind is limited by the onset of the mirror, cyclotron, and firehose instabilities, which grow increasingly sharp as $\beta_{\| \mathrm{p}}$ increases (Kasper et al. 2002, 2003). The image on the right shows the average value of $\mathrm{T}_{\mathrm{p}}$, indicating that the plasma is heated anisotropically to bring it near the instability thresholds (Maruca et al. 2011). Closer to the Sun $\beta_{\| p}$ is lower, so the allowable range of $R_{p}$ should be larger, but the heating may also be stronger. Statistical studies such as these, drawn from a large ensemble of measurements, are a power tool to identify the relative roles of heating and instabilities in modifying the VDFs (Bale et al. 2009). A statistical determination of the effects of instabilities within $0.25 \mathrm{AU}$ requires a large ensemble (at least several million) of solar wind measurements over all conditions near the Sun; since the growth rates of the instabilities are small compared to the ion gyro-frequency time resolution is not a driver; ion and electron temperature anisotropies measured to $20 \%$ accuracy. 
SWEAP measurements will be combined with electric and magnetic field measurements to identify the dominant heating modes in the solar wind as a function of distance from the Sun and solar wind type. These results will be combined with our theoretical models to determine how the solar wind is heated and to predict the mechanisms at work in the solar corona. The relative role of instabilities, dissipation, Coulomb relaxation, and adiabatic expansion in modifying ion and electron VDFs will be determined.

\subsubsection{Acceleration and Transport of Energetic Particles}

The third SWEAP science objective is to explore mechanisms that accelerate and transport energetic particles. The solar corona and the solar wind often contain diverse energetic charged particle populations accelerated by disturbed electromagnetic fields near the Sun. Solar energetic particle (SEP) acceleration sites include coronal mass ejections (CMEs), solar flares, and resonance with in situ waves and turbulence. SWEAP will help address these objectives in concert with ISIS measurements of the SEPs (McComas et al. 2014) through three observational goals: (1) Understand particle acceleration by CMEs and interplanetary shocks, (2) Understand the acceleration and transport of particles from solar flares into the solar wind, and (3) Determine if stochastic in-situ acceleration and energetic particle transport is significant.

(1) Understand Particle Acceleration by CMEs and Interplanetary Shocks CMEs are associated with some of the most intense gradual SEPs and intense Type-II radio emission due to energized electrons. Solar wind measurements are essential to understanding the evolution of the CMEs, the nature of CME-driven shocks near the Sun, and the mechanisms whereby electrons create Type-II emissions. Coronal mass ejections often drive strong fast shocks as they expand into the corona and interplanetary space and these shocks are believed to be responsible for the SEPs. The dominant acceleration mechanism at these shocks is likely either shock drift acceleration (SDA) or diffusive shock acceleration (DSA) depending on what the shock conditions are close to the Sun. Measurements of the solar wind conditions across shocks will determine basic shock properties such as orientation, Mach numbers, wave-speeds, compression ratios, levels of turbulence, and heating effects in order to distinguish between these models. DSA relies on power in the MHD turbulence at a quasi-parallel shock wherein Alfvén waves are amplified by streaming protons. To characterize shock acceleration SWEAP must: measure background plasma conditions and shock properties at the acceleration site, including shock thickness, shock compression ratio, and velocities; investigate waves excited by streaming protons ahead of shocks; and measure non-Maxwellian VDF features including beams within the foreshock and downstream sheath. Requirements: Measure plasma properties of at least one CME-driven shock within $20 \mathrm{R}_{\mathrm{s}}$ and more than five within $0.25 \mathrm{AU} ; 2 \mathrm{D}$ ion and electron VDFs at $2 \mathrm{~Hz}$ to resolve asymptotic conditions upstream and downstream of shock.

(2) Understand the Acceleration and Transport of Particles from Solar Flares into the

Solar Wind Solar flares can produce intense bursts of energetic ions, electrons and neutrons. Open questions include the time dependence of energetic particle production and the rates particles from flares diffuse across magnetic field lines. Energetic ions are observed in impulsive SEP events, but their precise origin is still presently unclear. SPP is in an excellent position to study this because the level of scattering should change dramatically as a function of distance from the Sun and the magnetic connectivity to source regions should be easier to establish. Figure 7 shows an example of energetic ions from a solar flare reaching 
Fig. 7 Suprathermal e-from 371-1370 eV (top) and the inverse speed of energetic ions (bottom) for 12-13 August 2000. Sudden drops in the e-flux, indicating loss of magnetic connection to the accelerating region, are associated with the disappearance of ions from the source (Chollet et al. 2009)

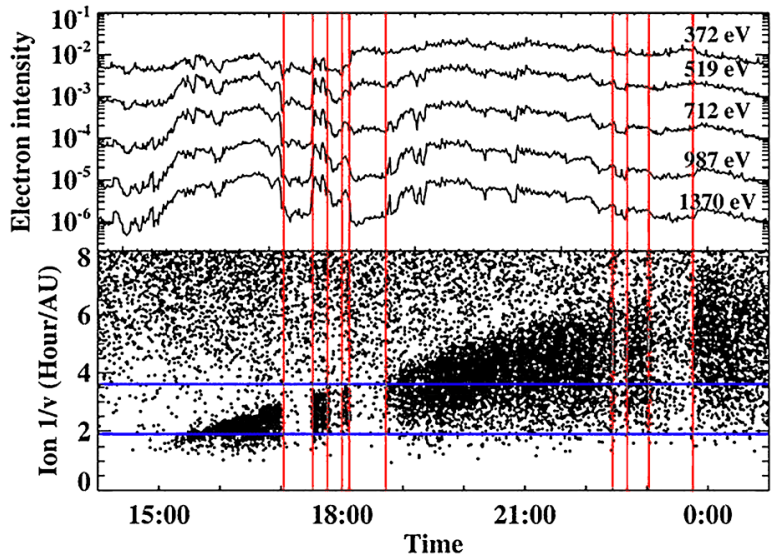

the ACE spacecraft at $1 \mathrm{AU}$ with an energy-dependent delay due to travel time. When the electron heat flux drops out, the spacecraft is not connected to the accelerating region and the ion signal also disappears. To address particle acceleration, SWEAP will (i) measure e-beams related to type III radio emissions; (ii) measure low-energy (up to $30 \mathrm{keV}$ ) SEP H and $\mathrm{He}$ ions close to the Sun and identify their source and acceleration site, including spectra, temporal profiles of particle fluxes, and PADs to identify suprathermal particle transport near the Sun (field-aligned or cross-field), and (iii) use BDEs to study magnetic field connection to the flare site. Requirements: electron beam spectra, pitch angle distributions in energy range 100-10000 eV; Detect SEP H and He ions with energy up to $20 \mathrm{keV}$ every 10 seconds for required dispersion resolution.

(3) Determine if Stochastic in situ Acceleration and Energetic Particle Transport Is Significant Ion populations observed during quiet times (i.e., in the absence of shocks and CMEs) often possess a suprathermal tail (Gloeckler et al. 2008), whose origin has been much debated. Close to the Sun, where pickup ion intensities are low and contamination therefore minimized, SPP may be able to discover the energization mechanism for the suprathermal tails. Two competing acceleration possibilities are (a) resonant statistical acceleration by waves, including quasi-linear 2nd order Fermi acceleration and transit-time damping (Fisk et al. 1974; Miller 1998), and (b) non-resonant stochastic acceleration by turbulent fluid compressions and rarefactions (Ptuskin 1988; Webb et al. 2003; Le Roux et al. 2002; Cho and Lazarian 2006; Fisk and Gloeckler 2006). Key measurements by SWEAP will include (i) suprathermal ion spectra; (ii) wave modes, density, and velocity vectors, amplitudes and polarization properties, and correlating with magnetic field observations; (iii) plasma turbulence, power spectra (up to the dissipation range of frequencies greater than the proton gyro-frequency, $\sim$ tens of $\mathrm{Hz}$ at $\sim 20 \mathrm{R}_{\mathrm{s}}$ ), the outer scale, wavevectors (slab/2D), cross-correlations between velocity and density, structure functions, helicity and cross-helicity (Roberts et al. 1987; Matthaeus et al. 1990; Bavassano et al. 2000), and (iv) the radial evolution of turbulent power and relevant length scales (Zhou and Matthaeus 1990; Zank et al. 1996) and identification of driving mechanisms (fast/slow stream interactions, shear, and compressions). Requirements: e-strahl: energy range: $70-1000 \mathrm{eV}$, angular resolution, $<10^{\circ}$; Bi-directional electrons: energy range, $\geq 80 \mathrm{eV}$; Pitch angle distributions in at least 10 energy channels, angular resolution: $20^{\circ}$; Detect ion halos with energy up to $20 \mathrm{keV}$. 
SWEAP measurements permit closure of open questions in energetic particle acceleration. The measurements described above will be combined with supporting electromagnetic field and SEP observations, leading to an understanding of particle acceleration processes close to the sun and with heliocentric distance. We will be able to understand and distinguish physics of impulsive and gradual events, identify mechanisms for quiet time particle acceleration, observe particle acceleration by turbulent reconnection, understand the seed particle population, and understand mean free paths, including perpendicular transport and field line wandering.

\subsection{SWEAP Science Requirements and Performance}

Section 1.1 established the science goals of the SWEAP Investigation, showed how they relate to SPP goals and objectives, and defined the measurements that would be required to achieve these goals. The most stringent measurement requirements were identified for each aspect of electron, alpha, and proton measurements. These driving requirements, along with the minimum anticipated capabilities for each of the SWEAP sensors are shown in Table 3.

We now elaborate on two of the major driving performance requirements for SWEAP: field of view and measurement cadence.

A major technical driver for SWEAP is the broad field of view requirement for ions and for electrons. The solar wind instruments on SPP must be able to measure the core of the proton VDF at least $90 \%$ of the time in order to successfully accomplish goals such as linking the solar wind to its sources, detecting shocks, and determining how the solar wind is heated. A significant obstacle for instruments on SPP is that the sunward fields of view for instruments in shadow are blocked by the heat shield. Figure 8 illustrates the portion of an ion velocity distribution function (VDF) that would be blocked by the heat shield for typical conditions at 39 and $9.5 \mathrm{R}_{\mathrm{s}}$. Far from the Sun, an instrument such as SPAN-A on the ram side of the spacecraft cannot see the core of the ion VDF because of the obstruction of the heat shield. Instead, the SPC view of the Sun is needed in order to measure the ions. Closer to the Sun, the solar wind ion flow is still nearly radial on average, but the orbital motion of the spacecraft becomes large, approaching $200 \mathrm{~km} / \mathrm{s}$, and the ions appear to flow into the ram-facing side of the spacecraft. Only under those conditions close to the Sun is SPAN-A able to see the core of the ion VDF. It is very likely that close to the Sun plasma waves will become so strong that the ion flow angles will fluctuate rapidly and still require both the SPC and SPAN-A fields of view. When a portion of the central thermal width of a VDF is blocked by the heat shield it becomes very difficult to extract meaningful measurements of density, velocity, or temperature. Results from the Fast Imaging Plasma Spectrometer (FIPS) sensor on MESSENGER are a relevant example of the challenges encountered observing solar wind in the inner heliosphere on spacecraft with instruments behind heat shields (Raines et al. 2011).

It is also important to observe some electron properties from the sunward direction on the spacecraft. For the thermal portion of the electron VDF, electrons are subsonic, and therefore can be seen from any direction about the spacecraft. However the electron strahl, a higher energy component of the electron VDF that is the major source of heat flux in the inner heliosphere and must be tracked to understand heating of the corona and solar wind, can also be blocked by the heat shield. The electron heat flux is aligned with the local magnetic field, and close to the Sun the field will tend to be more radially aligned on average. Since the strahl electrons are moving significantly faster than the spacecraft they do not experience the aberration in flow that helps for viewing ions. Since Helios observed its angular width to shrink as the spacecraft moved closer to the Sun, the strahl will be even more difficult to detect by SPP. 


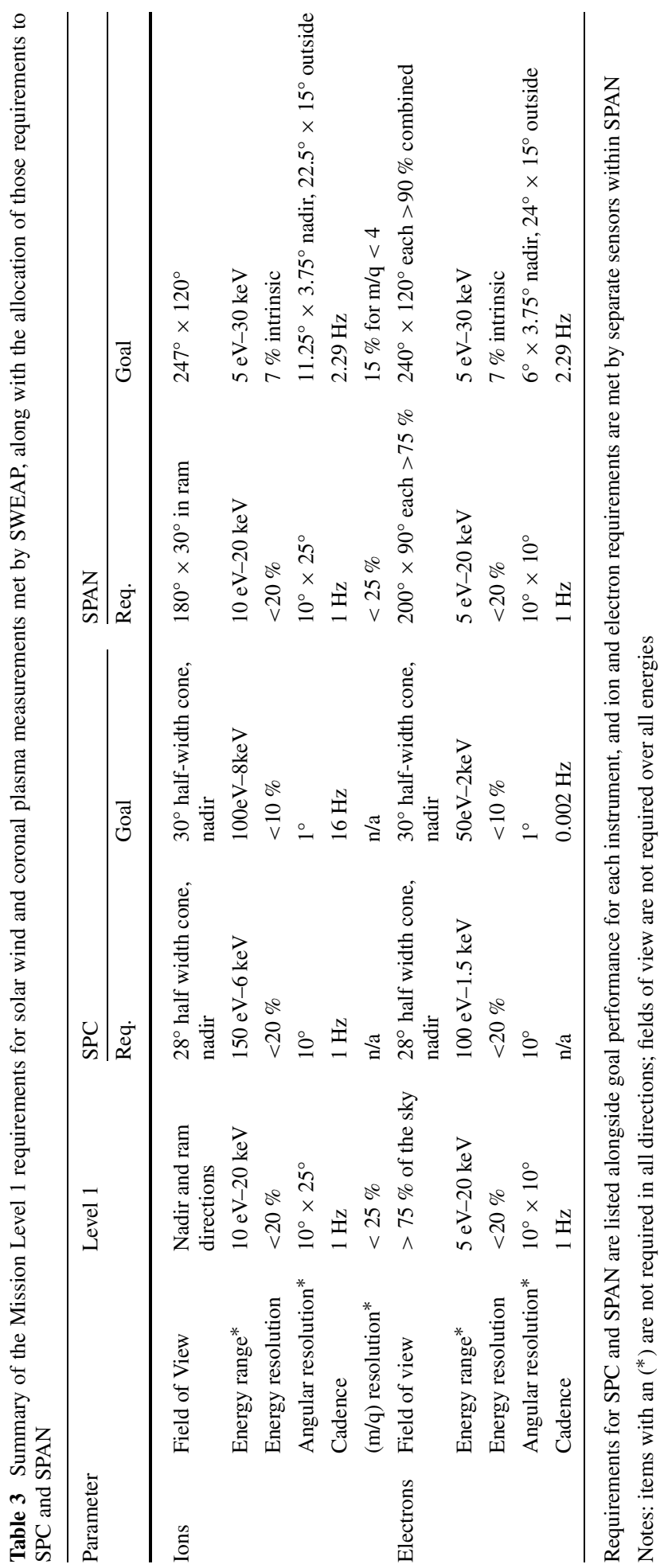


Fig. 8 Sketches of the portion of ion VDFs blocked by the heat shield using an extrapolation of ion properties and SPP motion to 38.4 and $9.5 R_{\mathrm{S}}$. The green region indicates the portion of the FOV for an instrument on the spacecraft bus that is blocked by the heat shield. The solid lines indicate contours in phase space for a representative proton VDF including a core and beam. The colored contour region indicates the portion of the proton VDF that SPAN can detect. The blue indicates the $28^{\circ}$ half-width FOV of SPC, which sees the entire VDF that would otherwise be blocked by the shield
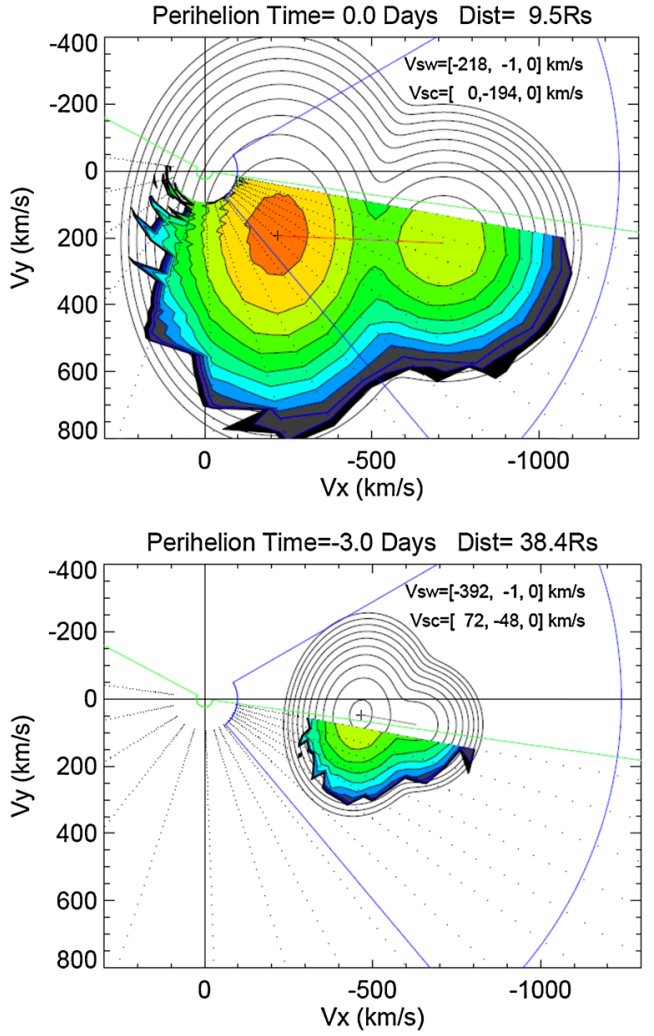

Observations by both SPC and SPAN are required to reliably detect the proton core and electron strahl within 0.25 AU due to the blockage of the SPAN field of view by the heat shield, radial variation in solar wind speed and flow angle fluctuations, and change in the orbital velocity of the spacecraft as a function of distance from the Sun and time in the mission. Monte Carlo simulations were conducted to determine the fraction of time that measurements by SPC and SPAN alone and combined as SWEAP were sufficient to detect the proton core and the electron strahl. For ions, protons are the driving requirement because all other species will either have the same velocity or drift at up to the Alfvén speed and be easier to detect. The simulation combined the SPP spacecraft trajectory and velocity with extrapolations of solar wind properties (magnetic field fluctuations and orientation, solar wind speed and velocity fluctuations, solar wind Mach number and thermal speed) based on fits to Helios observations with distance similar to Hellinger et al. (2013). For each simulated observation at a given distance from the Sun, the bulk velocity and thermal speed of the proton distribution was calculated in the reference frame of each instrument. To detect the proton distribution an individual instrument, or the combination of the two instruments, must be able to view at least one thermal width around the proton core. To detect the electron strahl the instrument must be able to view within $5^{\circ}$ of the magnetic field direction. Results of the simulation in Fig. 9 show the percentage of time along the final SPP orbit that SPC and SPAN alone and combined can detect the proton core and electron strahl. The resulting percentage of time SPP would detect proton core and electron strahl were then integrated from $9.5 \mathrm{R}_{\mathrm{s}}$ to $0.25 \mathrm{AU}$. SPC alone would be able to see the proton core $87 \%$ of the time and the electron strahl $71 \%$ of the time. SPAN alone would see the 
Fig. 9 The percentage of time SPC and SPAN can detect e-strahl (lower plot) and proton core (upper plot). The red and blue curves are for SPC and SPAN acting alone respectively and the green curve is the combined capability of the two instruments. The inbound and outbound portions of the orbit were considered separately (due to the different contributions of the orbital velocity of the spacecraft inbound and outbound) and are shown respectively as solid and dashed lines

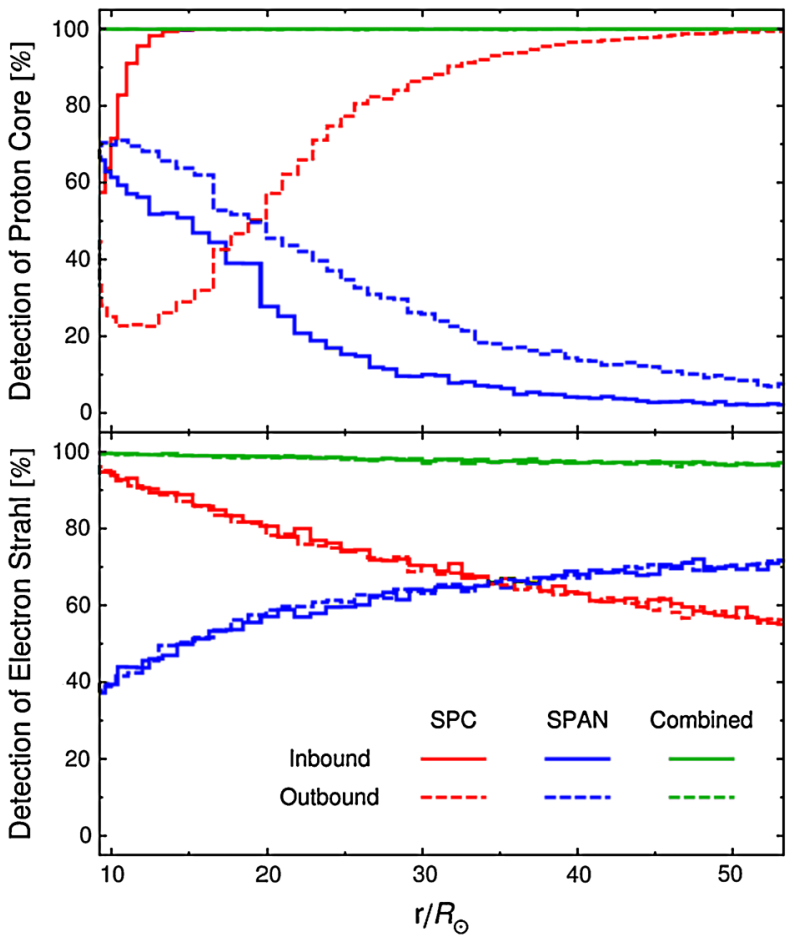

proton core $24 \%$ of the time and the strahl $62 \%$ of the time. Simulations of combined SPC and SPAN observations produce $100 \%$ recovery of the proton core and $98 \%$ coverage of the electron strahl. While this simulation uses a very specific set of assumption based on an extrapolation of Helios observations at larger radial distances from the Sun, it illustrates how sensitively our detection of basic solar wind parameters depends on distance from the Sun and our ability to view both the ram direction and the Sun.

To further illustrate the importance of the fields of view provided by the SWEAP instruments we convolved the simulations of proton core detection by the sensors with the spacecraft trajectory and statistical models for the expected occurrence rates of CME-driven shocks, HCS crossings, and magnetic clouds. For the occurrence of well-defined magnetic clouds we used the fact that PVO at 0.7 AU saw 75 events over ten years (Mulligan et al. 1998). For CME-driven shocks we used Helios observations of IP shocks as a function of distance and year and removed co-rotating interaction regions as they will not be seen within $0.25 \mathrm{AU}$ to produce an average rate of 14/year. To determine the number of encounters with the HCS we placed a simple tilted HCS on the solar surface rigidly rotating every 25 days and used to the SPP trajectory to identify the number of HCS crossings over the mission as a function of distance. Note that the occurrence rates of CMEs and magnetic clouds is a strong function of level of solar activity, and thus actual observations by SPP may be very different due to the different phases of the solar cycle observed by each mission, and due to the overall long term decrease in solar activity seen in the last thirty to fifty years. Given that activity is overall decreasing, these estimates are likely upper limits.

SWEAP provides a high probability that SPP will return solar wind measurements across shocks, CMEs, and the HCS, as shown in Table 4. In this table we compare our requirements 
Table 4 Mean expected number of times SWEAP instruments will observe different targets in the inner heliosphere and probability at $68 \%$ confidence level that SWEAP meets its goal. For comparison, the expected number and probability of success are given separately for SWEAP observations with both SPC looking at the Sun and SPAN-A looking in the ram direction, and just for SPAN-A alone. Clearly ion observations in both the sunward and ra directions significantly improve the scientific return of the plasma instruments

\begin{tabular}{|c|c|c|c|c|}
\hline \multirow[t]{2}{*}{ Goal at $68 \%$ confidence } & \multicolumn{2}{|c|}{ SPC and SPAN-A } & \multicolumn{2}{|c|}{ SPAN-A alone } \\
\hline & \# & Prob. & \# & Prob. \\
\hline At least one CME-driven shock within $20 \mathrm{R}_{\mathrm{s}}$ & 1.5 & $78 \%$ & 0.8 & $54 \%$ \\
\hline At least ten HCS crossings within $20 \mathrm{R}_{\mathrm{S}}$ & 19.0 & $98 \%$ & 9.5 & $36 \%$ \\
\hline More than one magnetic cloud within $0.25 \mathrm{AU}$ & 3.7 & $89 \%$ & 0.9 & $24 \%$ \\
\hline More than five CME-driven shocks within $0.25 \mathrm{AU}$ & 9.2 & $90 \%$ & 0.3 & $4 \%$ \\
\hline More than twenty HCS crossings within $0.25 \mathrm{AU}$ & 49.1 & $100 \%$ & 12.7 & $2 \%$ \\
\hline
\end{tabular}

for the number of different solar wind structures we require SWEAP to be able to detect within $0.25 \mathrm{AU}$ (at the $68 \%$ confidence level using Poisson statistics and the occurrence rates calculated as described above). For both SPC and SPAN and just SPAN we show the average number of each structure the instruments are expected to detect and the corresponding probability that they meet our requirements over the course of the mission. The odds of SWEAP measuring the plasma across more than five CME-driven shocks over the course of the mission is $90 \%$, while for SPAN alone it is only $4 \%$. This difference would significantly impact the ability of SPP to meet its goals related to particle acceleration in the inner heliosphere. We anticipate that SWEAP would see almost $50 \mathrm{HCS}$ crossings within $0.25 \mathrm{AU}$, while SPAN-A alone would see 13.

The second driving performance requirement for SWEAP is cadence. Since the convected ion gyro-scale within $20 \mathrm{R}_{\mathrm{s}}$ is likely in the range of $10-30 \mathrm{~Hz}$, some measurements at higher frequencies of $50-100 \mathrm{~Hz}$ are necessary to observe and understand solar wind heating. Since the telemetry rates for instruments on this mission are highly constrained, we address these cadence requirements through three measurement strategies. First, while the Solar Probe Cup generally uses 8-16 energy/charge windows to measure the peak of the solar wind ion distribution function we employ an occasional high cadence mode where the instrument remains in one energy/charge window and repeatedly measures ion flow angle and flux at cadences greater than $128 \mathrm{~Hz}$. As we will show below, our simulations suggest that a precision of $0.1^{\circ}$ change in flow angle at $128 \mathrm{~Hz}$ in a given energy/charge window is sufficient to detect high frequency wave-particle coupling for ions. Note that this precision requirement is distinct from the separate angular resolution requirement specified in Table 3. Detailed 3D velocity distribution functions with better than one second time resolution and wave-particle correlations at ion and electron kinetic scales are required to observe and characterize structures and wave-particle interactions in the corona and solar wind. Since telemetry rates on SPP are highly constrained, we place sufficient storage within the SWEM to run and archive SPAN observations at their maximum data rates throughout each encounter, and then downloaded high resolution data for regions of interest identified after the encounter and an initial examination of summary observations. Finally, to search for wave-particle interactions at the highest cadences, we employ a direct high speed interface between the SWEAP and FIELDS (Bale et al. 2015, this issue) instrument suites. Particle counts are relayed to FIELDS through the interface, and a wave-particle correlator calculates correlations and phase delays between particles and electromagnetic fields. 
Fig. 10 We will reach closure on the dissipation mechanisms that act in the solar wind through a combination of analysis of power spectra and numerical simulations. This figure illustrates expected turbulent spectra at $20 \mathrm{R}_{\mathrm{S}}$ based on theoretical predictions and extrapolation of Helios observations, along with the expected noise floor of the SWEAP/SPC instrument

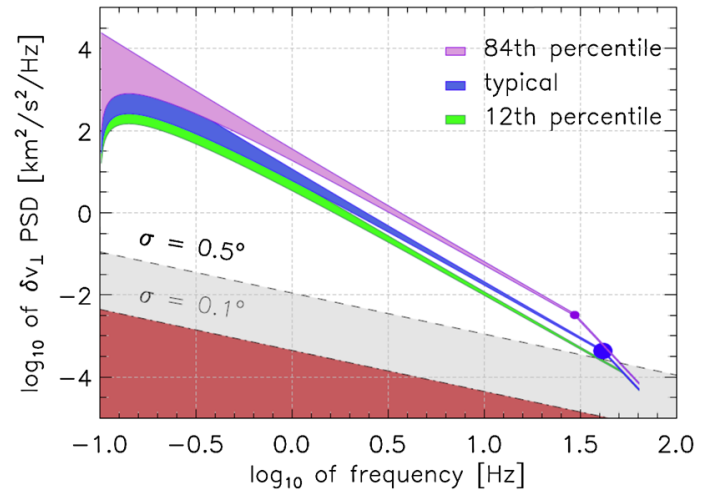

Our simulations of instrument performance show that the cadence strategy outlined above permits SWEAP to identify the heating and dissipation mechanisms in the solar wind. We have calculated various theoretical signatures of dissipation and fed the results into SWEAP instrument performance simulations to demonstrate that the SWEAP data products are sufficient to resolve the dominant mechanisms. The solar wind heating mechanism in Sect. 1.2 with the most stringent requirement on temporal resolution and flow angle accuracy is dissipation through turbulence. In this model, magnetic structures emerge and cascade down to smaller scales due to the nonlinear interaction between eddies, and the transition of the turbulent cascade between inertial and dissipative regimes is manifest as a broken power law in the power spectral density (PSD) of magnetic field and velocity fluctuations at the convected gyro-scale. Figure 10 illustrates our ability to detect both the spectrum of turbulence the break-point in the power law for a range of solar wind conditions. For this figure we constructed three estimates of the shape of the PSD near the Sun extrapolating from Helios observations to calculate the 16th, 50th, and 84th percentile estimates of the breakpoint frequency and PSD amplitude. For each of these spectra, the PSD was transformed to the time domain and resampled at the SPC $128 \mathrm{~Hz}$ burst flow angle measurement cadence. Figure 10 demonstrates the outcome of this procedure with SPC in $128 \mathrm{~Hz}$ burst proton flow angle mode at a distance of $20 \mathrm{R}_{\mathrm{s}}$, assuming a measurement error of $0.1^{\circ}$ degrees (expected) and $0.5^{\circ}$ degrees (worst case) and a total integration time of $10 \mathrm{~s}$. In this figure, the measured PSDs are shown with violet, blue, and green bands. Fits to the spectral break are indicated with filled circles that are scaled to the uncertainty. For the majority of cases the spectral break is easily resolved.

\section{SWEAP Overview}

\subsection{Suite Philosophy}

The overall organizational structure of the SWEAP Investigation at the time of Preliminary Design Review, including key personnel and responsibilities, is illustrated in Fig. 11. The investigation is managed by the Smithsonian Astrophysical Observatory (SAO). In addition to the management of the suite, SAO is also responsible for the SWEAP Science Operations Center (SOC) and the Solar Probe Cup (SPC). The University of California, Berkeley Space Sciences Laboratory is responsible for the Solar Probe Analyzers (SPAN) and the 


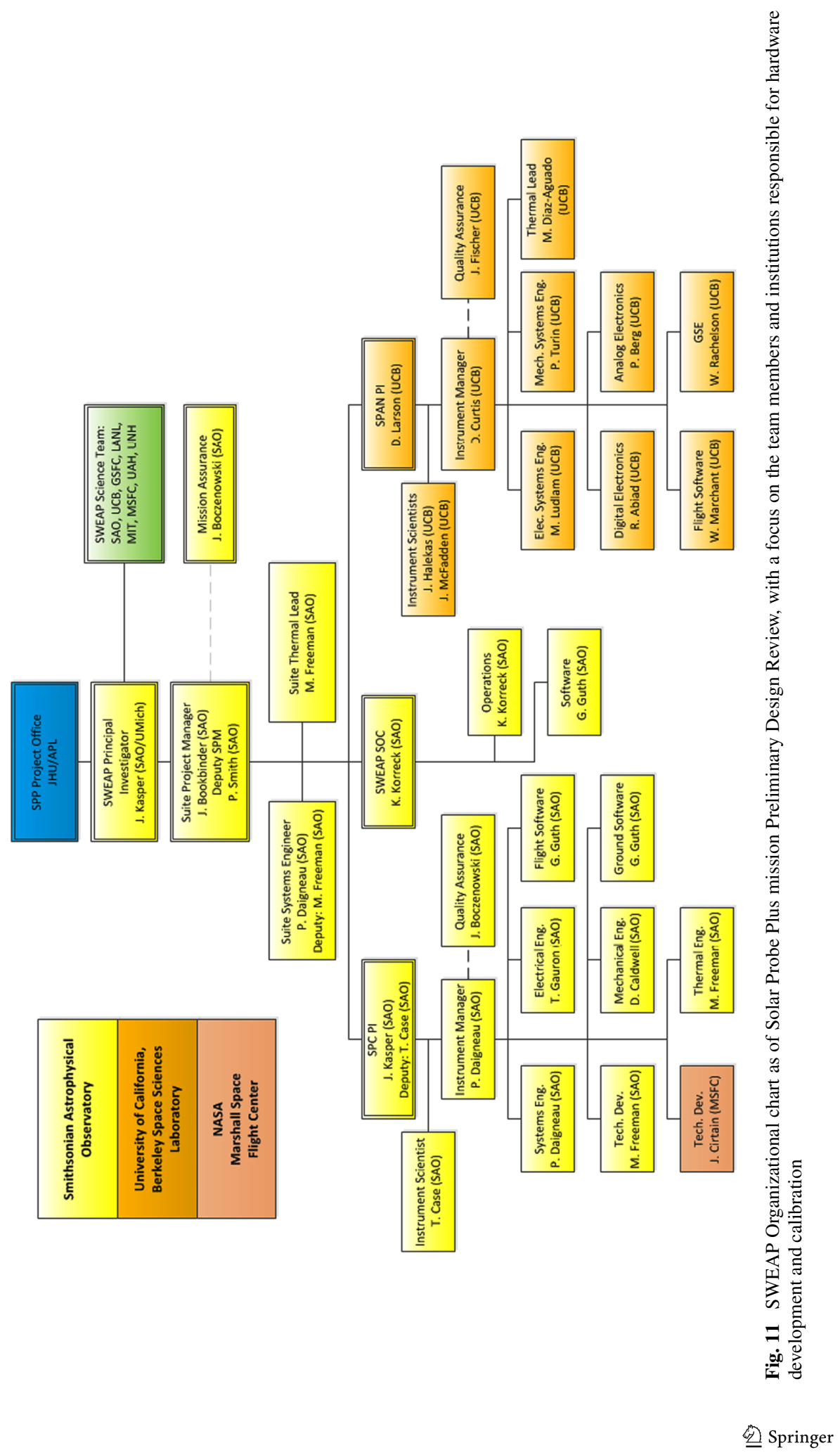




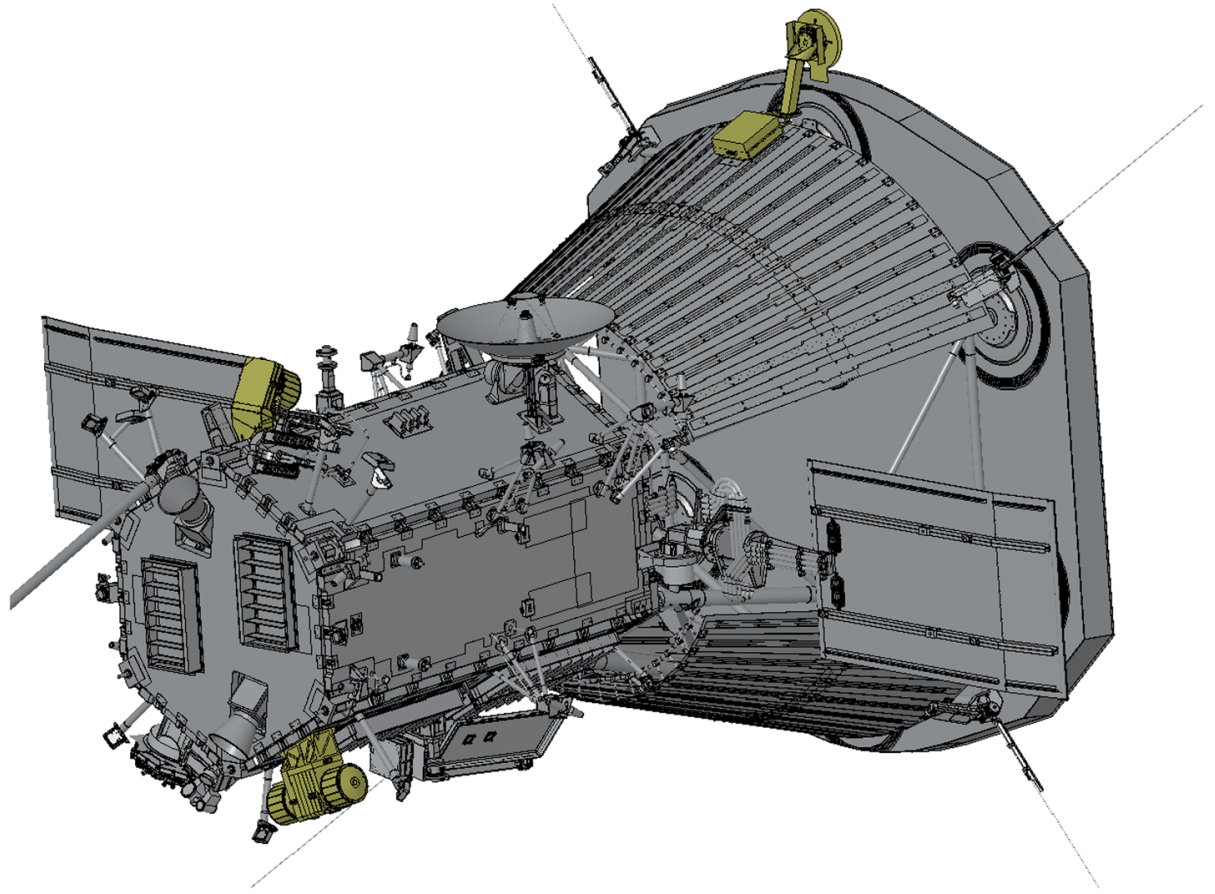

Fig. 12 Placement of the SWEAP instruments (highlighted in yellow) on the Solar Probe Plus spacecraft. See the mission review by Fox et al. (2015, this issue) for a detailed description of the spacecraft. This view is from the perspective of an observer North of the ecliptic plane looking down on the spacecraft. In this view the spacecraft is oriented with the Sun-facing thermal protection system (TPS) heat shield on the right, the ram-facing (direction of motion about the Sun) side of the spacceraft looking down, and the anti-ram side looking up. SPC is seen in the upper right side of the drawing, looking around the edge of the TPS. SPAN-B is seen in the top left side of the drawing. SPAN-A is visible in the lower left side. The SWEM is internal to the spacecraft bus and not shown in this visualization, but is located about halfway up the hexagonal spacecraft bus on the same panel as SPAN-B

SWEAP Electronics Module (SWEM), which controls the instruments, distributes power, formats SWEAP data products, and serves as the single interface to the spacecraft. Additional science team members are from institutions in the United States and France including the University of Michigan, NASA Marshall Space Flight Center, NASA Goddard Space Flight Center, University of Alabama Huntsville, Los Alamos National Laboratory, Massachusetts Institute of Technology, and the University of New Hampshire. SPC is described in Sect. 3 and SPAN is described in Sect. 4. Suite commissioning, operations plans, and data products are described in Sect. 5.

\subsection{Suite Placement and Fields of View}

Figure 12 illustrates the current mechanical design of the Solar Probe Plus spacecraft, along with the placement and orientations of the SPC, SPAN-A, and SPAN-B instruments, which are highlighted. In this image the spacecraft is oriented with the heat shield on the right and the spacecraft bus on the left, the ram-facing side of the spacecraft is pointing down and the anti-ram side of the spacecraft is pointing up. SPC can be seen on the edge of the heat shield with a small suite-provided strut that allows the sensor of the instrument to face the Sun 


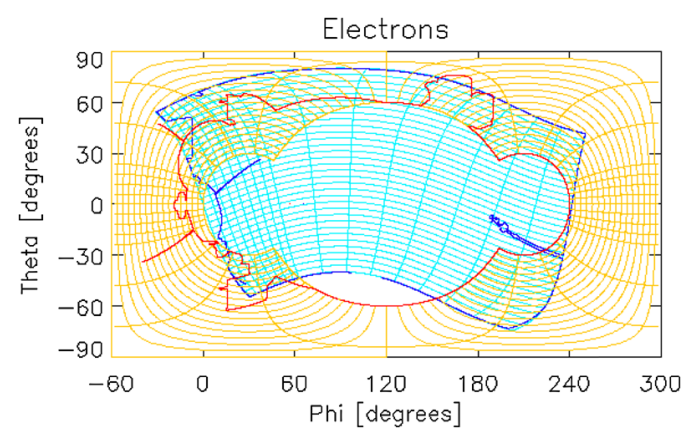

Fig. 13 Electron FOV of the instruments, showing combined anode/deflection angle maps for electron energies up to $4.5 \mathrm{keV}$ (blue $=\mathrm{SPAN}-\mathrm{A}$, orange $=\mathrm{SPAN}-\mathrm{B})$. The $\left(0^{\circ}, 0^{\circ}\right)$ direction is sunward, and $\left(90^{\circ}, 0^{\circ}\right)$ points to the ram. Spacecraft obscurations are outlined in blue for SPAN-A and red for SPAN-B. The only portions of the sky not covered by one of the two sensors are those directly sunward (blocked by heat shield) and the small surrounding region $\left(110^{\circ},-50^{\circ}\right)$. The portion blocked by the heat shield is visible to SPC up to electron energies of $2 \mathrm{keV}$ (the 29 degree half-width FOV of SPC is not shown on this figure)

while the high voltage and signal detection electronics sit in the shadow in the small box at the end of the strut. SPAN-A with its two electrostatic analyzers (electrons and ions) is visible on the lower left side of the image on the ram facing side of the spacecraft. SPAN-B, with its electron electrostatic analyzer, is visible on the upper left side of the image. Not seen in this image is the SWEAP Electronics Module (SWEM), which is embedded within the spacecraft bus.

From these locations the instruments are able to detect electrons over almost the entire sky, and ions from within thirty degrees of the Sun and from the ram direction. The SPC strut is designed to place the instrument sufficiently close to the front of the spacecraft heat shield that there are no obstructions to its field of view, which is a 60 degree full-width cone in the sunward direction. The SPAN electron fields of view and planned angular pixels are shown in a two dimensional projection of the sky in Fig. 13. This presents the SPAN-A field of view and pixels as the blue contours, the SPAN-B field of view as the yellow contours, and the outline of the spacecraft, as seen from SPAN-A, as the white trace. See how SPANB observes electrons over the region of sky blocked by the spacecraft for SPAN-A. Higher angular resolution in the SPAN pixels is determined by the size of anode detectors within the instruments. Regions of higher angular resolution are oriented to provide the best chance of resolving the electron heat flux when the solar wind magnetic field is in the standard Parker spiral configuration in the ecliptic plane. The fields of view of the SPAN instruments are unobstructed except for minor features including the FIELDS antennas, the FIELDS magnetometer boom, and the solar panels. Note that as the spacecraft approaches the Sun the solar panels move closer to the spacecraft and further from the SPAN fields of view. Adaptive masks will be used to discard pixels too close to the solar panels from processing of electron observations for science products.

\subsection{Suite Electrical Interface and SWEM}

The SWEAP electronics module (SWEM) is the data and power interface between the SPC and SPAN instruments and the spacecraft. It provides intermediate data processing (moment calculations, mode switching, data compression and data storage) for the SWEAP data as well as centralized power conditioning. In general, the SWEM acts as the SWEAP suite 
central processing unit, housing command scripts for sensor initialization and configuration, high voltage control, and attenuator actuation and accumulating data into various packets for inclusion into the data stream to be transferred to the SPP solid state recorder (SSR).

The SWEM houses most of the spacecraft interface electronics for SWEAP in a single box to reduce mass, to simplify harnessing and interfaces, and to reduce duplication of interface logic, power converters and other common services. SWEM allows for a single interface to the spacecraft DPU, easing integration and test activities later in the project. The module is composed of 2 separate approximately $6 \mathrm{U}-\mathrm{VME}$ sized boards $(160 \times 200 \mathrm{~mm})$ : the Data Controller Board (DCB), and a Low Voltage Power Supply Board (LVPS).

The SWEM derives its design from THEMIS, HESSI, STEREO, and MAVEN, and makes extensive use of the technical capabilities demonstrated in the Instrument Data Processing Units (IDPUs) developed for these missions. The suite data interface and control design is derived from the MAVEN DCB board, modified slightly to incorporate the different SPP interfaces. The Coldfire processor implementation was qualified for use by MAVEN, and the design will be copied for SWEAP. The remaining circuitry needed on the DCB and LVPS are standard designs used extensively in previous missions.

Data Controller Board (DCB) This board receives commands and timing signals from the SPP DPU, and generates initialization and configuration messages for the SPANs and SPC. It acts as a router during data collection and provides intermediate processing such as on-board moment, pitch angle and averaging computations. Upon low voltage turn-on of the SPC and SPAN, the DCB will configure the sensors, enable the low voltage line that powers the high voltage supplies and execute the HV ramp-up commands that bring the supplies to nominal voltage. The DCB includes hardware and software safety latches to prevent accidental high voltage turn on. Attenuator position is controlled by the DCB, which also compresses the data into averages and calculates onboard moments, implemented in a single FPGA/embedded processor. The DCB FPGA also provides the SWEAP to SPP DPU interface, controlling the flow of raw data through the system. It receives commands from the SPP DPU processor and manages the SWEAP suite. Following the RBSP and MAVEN architecture, the DCB has memory on-board (rad-hard and SEE tolerant $10 \times 8$ GB Flash Memory that is the same as is currently flying on MAVEN) and takes formatted packetized data from the instruments directly making them available when requested for transmission to the ground without further processing. Support circuitry on this board includes address/data demultiplexing and an ADC for housekeeping data.

Flight Software The core of the SWEM DCB is a Coldfire processor implemented in an FPGA, as an IP-core. The Coldfire processor was used on MAVEN and builds on heritage code that has flown on many missions. This processor and the board electrical design was developed for UCB's Instrument package on RBSP, allowing SWEAP to take advantage of both hardware and software heritage.

The SWEAP FSW architecture uses dedicated FPGAs for routine data and memory management, instrument interfaces, and other repetitive tasks in order to leave the processor free for specialized duties and less frequent, higher level functions. Instrument housekeeping and commanding is implemented via low speed serial data interfaces in a point-to-point architecture. Instrument science data is relayed over separate, higher speed serial lines to the DCB where data is directly fed into on-board memory, formatted as packets, and sent to the $\mathrm{S} / \mathrm{C}$ mass memory for eventual downlink transmission. There are no time intensive tasks and CPU usage as a fraction of total available resources is low. Computation intensive tasks 
are performed in the instrument FPGAs using dedicated HW, the CPU only supports their operation.

Low Voltage Power Supply Board (LVPS) The SWEM LVPS generates the various voltages required by the SWEAP instrument suite. Regulated voltages are Pulse Width Modulated (PWM) and regulated to better than $\pm 5 \%$. This board also provides current monitors for secondary voltages. Each power supply is current limited on its primary side and is galvanically isolated primary to secondary. The input from the $28 \mathrm{~V}$ spacecraft power is soft started and filtered to meet EMI requirements.

\subsection{Science Data and Operations}

The Suite is operated through the SWEM. The science data is taken along with calibration sequence run according to a command plan uploaded and then executed by the SWEM. Details of the suite operating modes and data products produced by the SWEM are described in Sect. 5.

The SWEM has a number of independent operating configurations, which mainly affect instrument science data accumulation rates. Only two basic modes (Safe and Science Mode) affect power consumption and dissipation. In Safe Mode, only the core systems are powered on. Safe Mode is the power on condition, with only the core power and processing system powered on (the instrument sensors are not powered on). Science Mode is the normal operating state. In this mode, SWEAP is ready for full science data collection and is autonomously controlled using table driven mode configurations. In Science Mode, sensor data is sent to the SWEM at a constant rate. While the specific contents of SPC and SPAN data are different, all communication between the SWEM and instruments is accomplished through the standardized CCSDS protocol. Upon receipt of instrument data the SWEM makes use of FPGA-based logic to steer incoming data to the assigned memory locations in real-time based on programmable tables. Once in memory, the FPGA logic moves data through the data processing and compression pipeline, completes the data packetization, inserting ApIDs, and queues the data for transfer to the spacecraft.

Operation of SPC by the SWEM is a simple matter of uploading operating parameters to the SPC FPGA. The FPGA will then control the high-voltage modulator board and record telemetry that is then sent back to the SWEM. The SWEM contains a number of pre-programmed configurations to place SPC into the various combinations of ion, electron, peak-tracking, full-sweep, flux-angle and calibration modes. SPC sends 1 science packet per second to the SWEM.

The SPAN sensors are powered on via command from the SPP DPU to the SWEM. The SWEM contains command scripts for SPAN sensors to initialize the LV electronics and place the sensors in a default test mode, enable HV and bring HV to programmable levels, run various test sequences that confirm proper operations, and command the sensor into an instrument mode. The SPANs always make measurements at the same rate, but SWEM can load new high voltage lookup tables and/or trigger a switch between the operative high voltage table in the SPANs, which affects the energies and angles that a SPAN sensor scans in each measurement cycle. SWEM can also load and/or trigger a switch between the lookup tables that determine how counts are accumulated and binned in the SPAN sensor FPGAs. The combination of high voltage and product lookup tables defines the instrument operation, with the resulting sensor operational modes described in Sect. 5.1. 


\section{The Solar Probe Cup (SPC)}

\subsection{SPC Overview}

SPC is a Faraday Cup (FC) with a $60^{\circ}$ FOV (full-width) that views the Sun from the edge of the SPP heat shield and measures the reduced distribution function (RDF) and flow angles of ions and electrons as a function of energy/charge at high frequency. SPC measurements are essential for SPP and SWEAP because otherwise the solar wind would often be blocked by the heat shield. FCs measure the current produced on a metal plate by charged particles with sufficient energy/charge to pass through a grid placed at a variable high voltage (HV). The underlying technology is straightforward and similar to the operating principles of vacuum tubes. SPC filters charged particles based on the component $\left(E_{\|} / q\right)$ of their energy/charge parallel to the instrument line of sight. For a given $E_{\|} / q$, particles with any $E_{\perp} / q$ can enter SPC as long as their flow angle is within the FOV. In a single measurement the HV oscillates between two voltages. The population of plasma with $\mathrm{E}_{\|} / \mathrm{q}$ between these two voltages produces an AC current on the plate, and electronics isolate the AC signal and record it. SPC measures the currents produced on a circular plate divided into four quadrants and placed behind a smaller circular aperture. A cross section of the SPC sensor and an illustration of the measurement process is provided in Fig. 14.

The combined currents are directly related to the RDF, and ratios of the currents recorded by the different collector plates can be solved for the precise flow angle of the plasma as a function of energy/charge. Detailed properties of the solar wind such as velocity, density, and anisotropic temperatures are then determined by convolving a model velocity distribution function with a detailed instrument response function and deriving the best fit solar wind parameters (e.g. Kasper et al. 2006). The AC detection process makes SPC insensitive
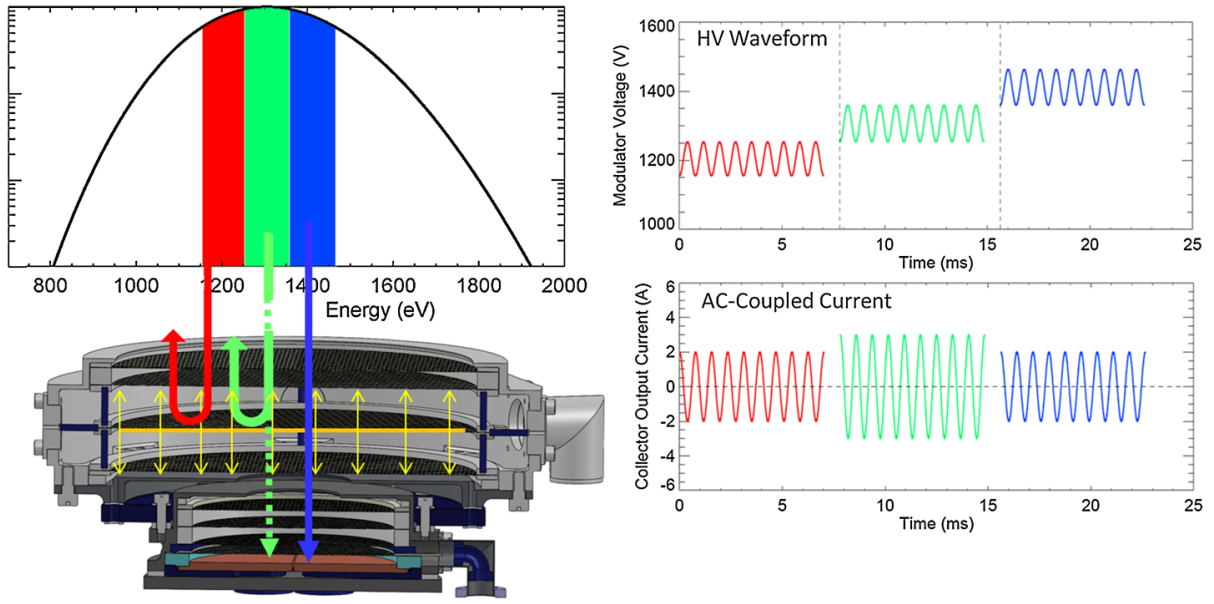

Fig. 14 Upper-left: a distribution function showing three voltage windows highlighted in different colors. Lower-left: Cross-section of Faraday cup Sensor Unit (FSU). The upper-section with a larger diameter is the 'modulator assembly' with the high-voltage modulator grid highlighted in orange. The electric field set up between the modulator grid and the adjacent ground grids is shown as yellow arrows. As the modulator grid in the FSU oscillates between two voltages (upper-right panel), only particles with energy/charge between the voltages produce an AC current on the collector plates (lower-right panel). These currents are amplified and fed to a synchronous detection circuit 
Fig. 15 Data from the Wind spacecraft illustrate how the noise level of a Faraday Cup that operates in a synchronous detection mode, such as SPC, does not change with the level of energetic particle flux

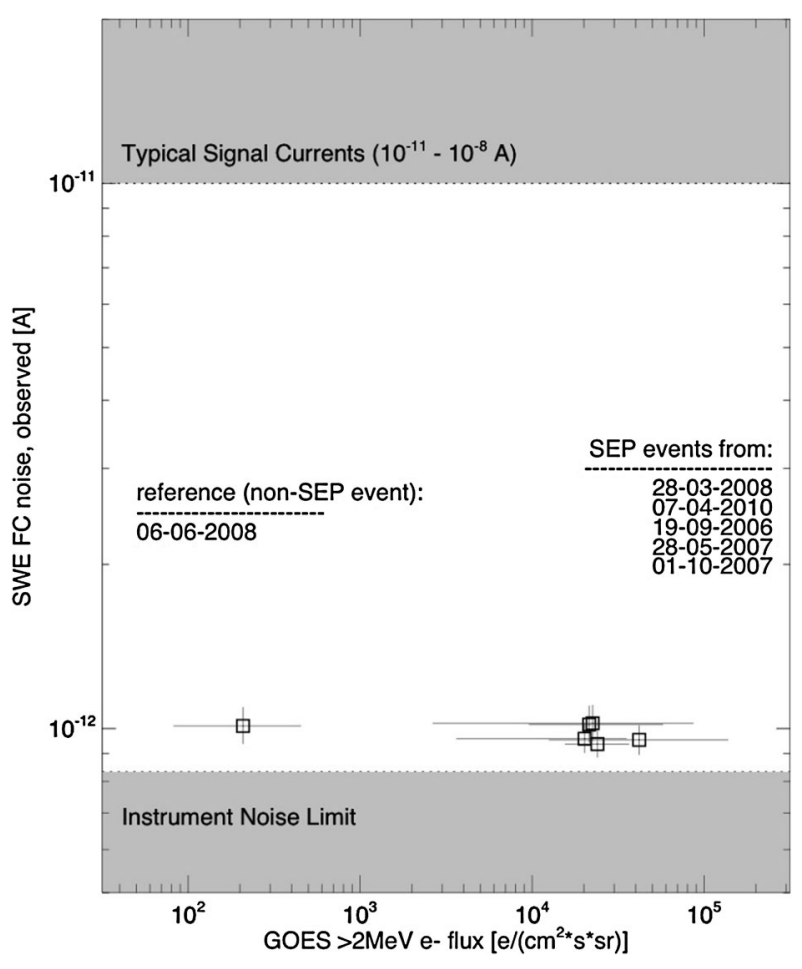

to lower-frequency noise sources, such as thermionic emission from hot surfaces, photoelectron emission from surfaces exposed to sunlight, and penetrating radiation from intense energetic particle events. Figure 15 shows data from the Wind/SWE Faraday cup (Ogilvie et al. 1993). The figure compares 5 large solar particle events to a quiet-time observation of the typical noise current in the FC. These observations indicate a lack of any dependence of the FC noise on the flux of penetrating radiation. This is to be expected, since any ionizing radiation simply produces a constant signal that is filtered out by the AC detection proves.

The FC has been a workhorse for space plasma measurements from the beginning of space exploration (Vasyliunas 1971; Gloeckler 1990). FCs have flown on Explorers 10, 18, 21, 28, 33, 35, 47 and 50, SOLRAD A and B, Pioneer 6 and 7, Mariners 3, 4, 5 and 10, OGO-1 and OGO-3, Voyager 1 and 2, and Wind. FCs have played a major discovery role in exploring space, from the first observations of ion fluxes in space (Gringauz et al. 1960) through the first observations of the termination shock (Richardson 2007). The FCs on Voyager 2 and Wind are still operating and providing invaluable solar wind measurements. The FC components and measurement process both function in a high temperature environment. Since the sensor is simply a metal plate, the response of the instrument is stable with time. The FCs on Wind have a drift in response of about $0.01 \% / y e a r$ (Kasper et al. 2006).

A significant effort has been expended in an assessment of materials for SPC, solar furnace testing of materials in an optical, thermal, and radiation environment well in excess of the closest SPP solar encounters, design and fabrication of a prototype, and successful operation a prototype instrument in a flight-like environment. Thermal and optical simulations described in Sect. 3.2 predict that SPC temperatures at closest approach will reach over $1600{ }^{\circ} \mathrm{C}$. Standard aerospace materials at these high temperatures can evaporate or outgas, especially when sputtering of solar wind ions and electron beam heating are factored in, 
Fig. 16 Layout of SPC alongside the spacecraft with key components labeled

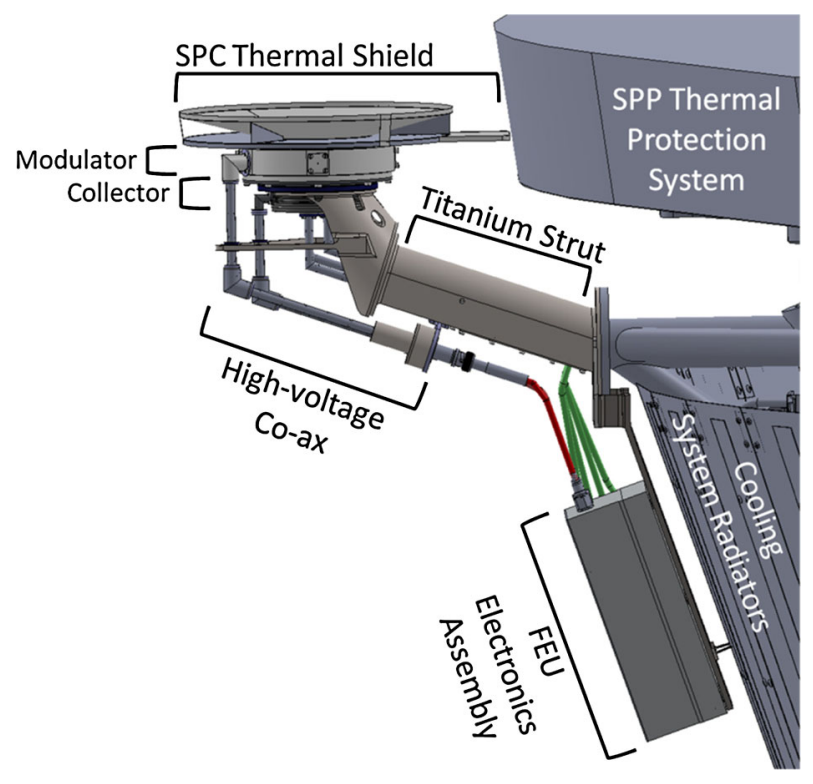

leading to changes in thermal/optical properties and degrading performance. Due to the Sunexposed materials at high voltage, we must also factor in thermionic emission due to both the hot cathode and field emission mechanisms, along with photoelectron induced emission hundreds of times more intense than generally seen near Earth. For the grids, we found the best materials to withstand the harsh conditions are high purity tungsten (W). Tungsten has a high work function and the highest melting temperature of the refractory metals, and is also simple to etch into grid patterns. Other materials employed in the sensor include pure Niobium, and an alloy of molybdenum, titanium and zirconium called TZM. The niobium is employed in the heat shield and in wires due to its flexibility and the ease with which it can be laser welded. The TZM was selected mainly because it is able to retain its strength at high temperatures and due to the relative ease with which it can be machined. Sapphire has emerged as the preferred material for insulating surfaces due to its high electrical resistivity at high temperatures combined with its relative mechanical strength compared to other materials such as pyrolytic Boron Nitride.

A preliminary description of the SPC design leading up to SWEAP Preliminary Design Review was presented in Case et al. (2013). SPC is divided into several smaller units, which are described here and shown in Fig. 16. The FC Sensor Unit (FSU) is the actual FC sensor that is exposed to sunlight. Two annular niobium plates at the front of the FSU permit plasma to flow into the instrument while shielding the edges of the SPC from sunlight. The FSU is mounted on the end of a titanium strut that interfaces SPC to the SPP transition structure. The Faraday cup Electronics Unit (FEU) is located on a platform directly next to the interface point on the SPP transition structure. The FEU contains preamplifiers that amplify the signals from each of the four collector plates. An FPGA and analog-to-digital converter (ADC) in the FEU digitize the amplified waveforms and perform the synchronous detection algorithm. The digitized currents are sent from the FEU to the Analog Processing Board (APB) in the SWEM, where further calculations are performed and the resulting packets are sent to the spacecraft solid-state recorder. The FEU also contains the High-voltage Modulator Board (HMB), which is driven by the FPGA and generates the High Voltage (HV) waveforms that drive the HV modulator grid within the FSU. The FEU is within the shadow 
Fig. 17 Cross-section of SPC with key components labeled

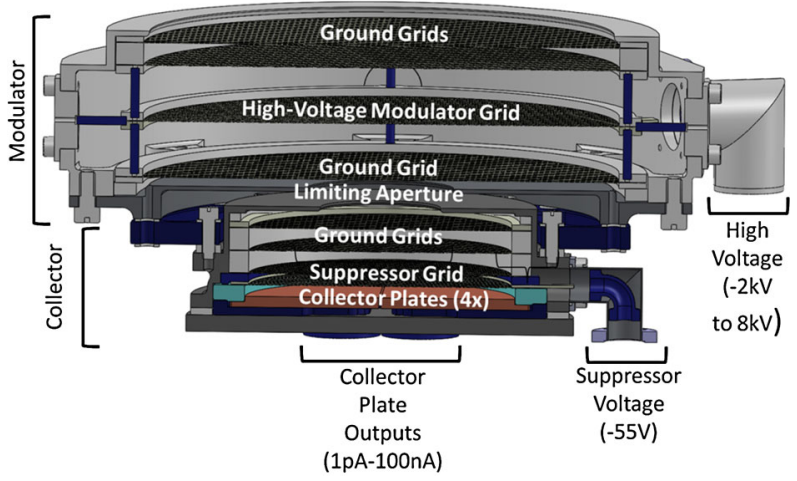

of the SPP heat shield, allowing for a relatively benign operating environment, with maximum temperatures expected in the electronics box to be approximately $70{ }^{\circ} \mathrm{C}$.

A cross section of the FSU is shown in Fig. 17. The modulator assembly filters particles by energy/charge and the smaller collector assembly records the flux and flow angles of the particles. Plasma enters the FSU through the large circular entrance aperture at the top of the instrument and enters the modulator assembly. The entrance aperture is designed to be sufficiently large so that the smaller limiting aperture between the modulator and collector assemblies is always fully illuminated by plasma over the entire range of angles of incidence. The limiting aperture is sized to detect the minimum flux with sufficient signal to noise, including the drop in signal strength due to the transparency of the grids. Fine conducting grids with high transparency throughout both assemblies act to filter particles and shield stray electric fields. After the entrance aperture a grounded grid prevents stray fields from leaving the instrument to ensure compatibility with electromagnetic instruments on the spacecraft. Within the modulator are three more grids: a HV modulator grid with a time-varying retarding potential sandwiched between two grounded grids. The particles that pass through the limiting aperture then produce a current on one of four $90^{\circ}$ wedge-shaped metal collector plates. The collector plates are larger than the limiting aperture, so an incident beam falls entirely on the collector plates. Three plates are sufficient to solve for flow angles, but a fourth plate provides an additional level of redundancy. A suppressor grid is maintained at $-55 \mathrm{~V}$ immediately above the collector plates. The suppressor reflects secondary electrons kicked off the plates from ion impacts back onto the plates and prevents low energy electrons from thermionic and photoelectric emission at the front of the FSU from reaching the plates, although this is not necessary because they do not produce an AC signal. The currents from the four plates are fed through coaxial cable to the FEU, where the measurement electronics amplify and digitize the waveform.

\subsection{SPC Estimated Performance}

The three key SPC performance parameters are energy resolution, signal to noise, and accuracy of angle measurements. Initial performance testing has been done with the SPC prototype and indicates that SPC will meet all of its high-level performance requirements. A twopronged testing approach was taken, with high-fidelity ion beam testing taking place at the Solar Wind Facility (SWF) at Marshall Space Flight Center (MSFC) and solar illumination testing taking place in a custom-built chamber at the Smithsonian Astrophysical Observatory (SAO). In order to provide some simultaneous testing, the SWF was fitted with a contact 
Fig. 18 Response of different energy windows to an ion beam of increasing energy

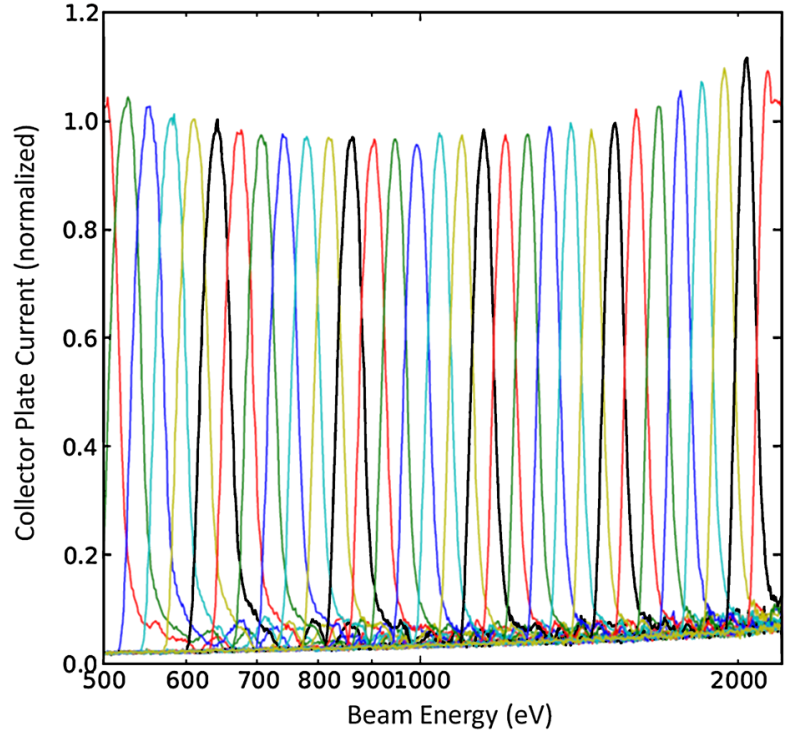

heater used to raise the temperature of the back of the collector housing and collector plates and the chamber at SAO was fitted with a lower precision ion source. Performance testing of the prototype in phase B demonstrated that SPC is able to meet all of its level-1 performance requirements. A detailed description of the SWEAP SPC technology development effort is documented elsewhere (Freeman et al. 2013).

To measure the energy resolution of SPC, a prototype was tested using the ion source at SAO. The instrument was run in a limited 'full-scan' mode, in which the current is measured in successive voltage windows by scanning in an upward direction and then again in a downward direction. In this case, the voltage windows are $7 \%$ wide and overlap by about $2 \%$. The energy of the beam is then incrementally increased in a slow manner, so that multiple measurements are made in each voltage window at each beam energy. The results are shown in Fig. 18. Voltage windows are shown as different colors (the colors repeat every 6th window). The peak of each voltage window is separated from the adjacent windows, showing that the instrument is able to resolve the energy of an ion beam to better than the separation of these energies windows (in this case $5 \%$ ). Further tests in phase $\mathrm{C}$ will further quantify the energy resolution of the instrument.

The angular response of the SPC prototype was measured using the SWF ion beam facility. A mono-energetic ion beam was produced that covered the full aperture of the SPC prototype. The instrument was then rotated through a range of angles while being exposed to this beam. Figure 19 shows the normalized current (in color) as a function of the angle of incidence of the ion beam and the energy window.

As the instrument is rotated, the ion beam appears in different energy windows because the modulator portion of the instrument affects only the parallel energy of the incoming ions. As such we expect a $\cos ^{2}(\theta)$ dependence of energy on incidence angle. The expected response is shown as a black line. The vertical green bar indicates SPC's field-of-review requirement. The beam is easily measured up to 10 degrees beyond the 30 degree requirement.

Testing was also performed to demonstrate the angular resolution of the instrument. Observations showed that the resolution is much better than 1 degree. This is confirmed by an analysis of the geometry of the cup and the measured noise performance of the measurement 
Fig. 19 SPC prototype response to a fixed energy beam at different angles of incidence

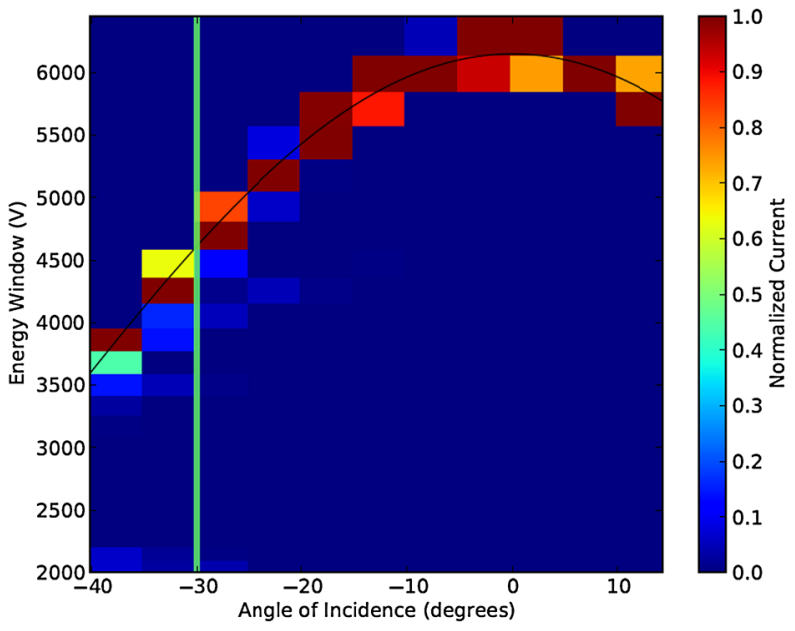

circuitry. Further testing in phase $\mathrm{C}$ will continue characterizing the angular response of the instrument.

\subsection{SPC Electrical}

The SPC Electronics Module is located at the base of the SPC strut and consists of a High Voltage Power Supply (HVPS), a Low Voltage Power Supply (LVPS), and a signal processing and control board referred to as the Faraday cup Electronics Unit (FEU). The HVPS provides the modulator and suppressor grid voltages. The modulation voltage is adjustable from $-2 \mathrm{KV}$ to $+8 \mathrm{KVDC}$ with an adjustable amplitude $50-800 \mathrm{~V}, 1280 \mathrm{~Hz}, \mathrm{AC}$ component. The high voltage $\mathrm{DC}, \mathrm{AC}$ component peak to peak voltage, and frequency are programmable via digital interface. The suppressor grid voltage is nominally -55 VDC. For each collector plate, the signal processing electronics first convert the input current waveform to a voltage waveform. This voltage waveform is amplified by four parallel gain stages, with the resultant waveforms are digitized by an ADC. The digitized waveforms are then routed to an FPGA that implements a discrete Fourier transform at the modulation frequency. The output of this algorithm is the peak-to-peak amplitude of the collector plate current at the modulation frequency. The gain-stage with the best SNR without saturating the amplifier is then selected and stored. The noise level of the system is about $5 \times 10^{-13} \mathrm{~A}$, and currents (including both alphas and protons) are expected to range between $5 \times 10^{-13}$ to $10^{-7} \mathrm{~A}$. Thus, the SNR in a single measurement will range between 1 and $10^{5}$. Protons, being the dominant species, will always have an SNR of more than 10.

\subsection{SPC Mechanical}

FSU materials and Fabrication Details of the SPC mechanical design, including structural considerations due to launch vibrations and thermally induced stresses has been documented elsewhere (Bergner et al. 2013). The dimensions of the FSU are directly derived from the desired sensitivity (the limiting aperture sized for the minimum expected current at $0.25 \mathrm{AU})$, range of observable flow angles $\left(56^{\circ} \mathrm{FOV}\right)$ and energy range (modulator sized to withstand $8 \mathrm{kV}$ ). The FSU was designed following standard practices for solar wind FCs 
Fig. 20 The four collector plates within their sapphire housing

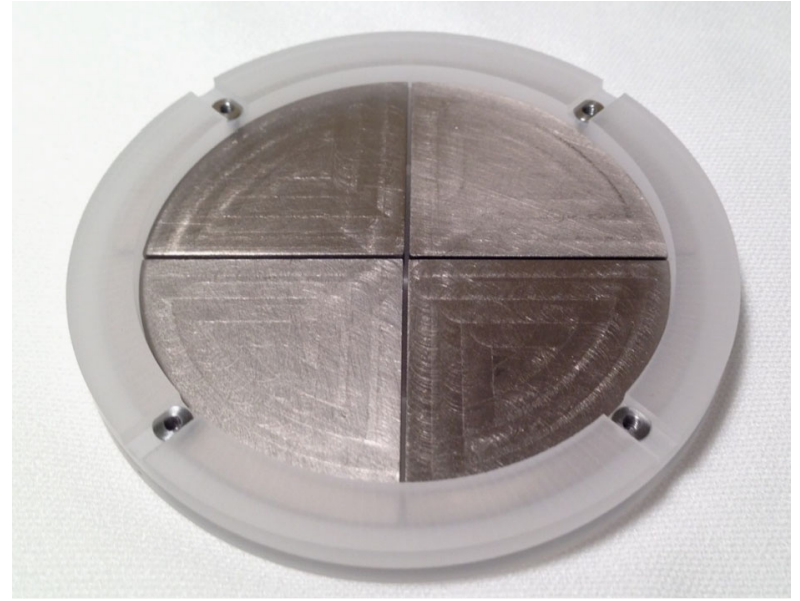

but with certain mechanical parts replaced with components made from materials more appropriate for higher temperatures. A stack of two thin annular niobium plates mounted to the front of the FSU limit direct exposure of the FSU and strut to sunlight while permitting access to the limiting aperture. The modulator and collector assemblies are machined from a high-temperature alloy called Molybdenum TZM (moly-TZM). The Delran insulators that have historically been used in Faraday cup to hold the HV grid in place and isolated from the modulator walls has been replaced with machined sapphire rods. The woven tungsten wire meshes used previously have been replaced with monolithic grids etched from single wafers of high purity W. We have found that these new grids are significantly stronger and more suitable for FCs than the old wire meshes, which were prone to failure through breaking wires and took weeks to manufacture.

Components are stacked within each subassembly, which is then sealed from the back with a TZM plate. The stack consists of niobium spacer rings, individual grids, and sapphire spacer rods and rings. High-voltage is transmitted to the modulator grid using a niobium wire fed from the side of the modulator assembly and bonded to a niobium annulus via crimping and laser-welding. The HV grid is then sandwiched between the niobium annulus and a tungsten annulus. The HV wire exits the side of the modulator housing and enters a custom hard coaxial cable. In that cable, sapphire tubes insulate the HV wire from a niobium outer tube, which is at ground. In the modulator assembly, ground grids make a firm mechanical and electrical connection to each other, to the ends of the assembly, and to the niobium spacer rings. This stacking technique makes assembly straightforward and permits mechanical flexibility in the presence of temperature gradients. See Fig. 17 for an annotated cross-section of the components in the sensor.

The structure of the collector assembly is similar, but ends with the four Niobium collector plates seated within a shallow sapphire cup. Signal wires are laser welded to the protrusions on the back of each collector plate that extend through the collector housing cap. Each signal wire then runs through hard coaxial cable (of similar construction as the HV coaxial cable) to the FEU. Figure 20 shows an image of the collector plate assembly which is the final component to be stacked into the collector housing. The four niobium collector plates sit on a bed of sapphire and are then clamped in place by a sapphire annulus that is held down by four bolts. In final stack-up, the suppressor grid would be placed directly against the top surface of the upper sapphire annulus. 


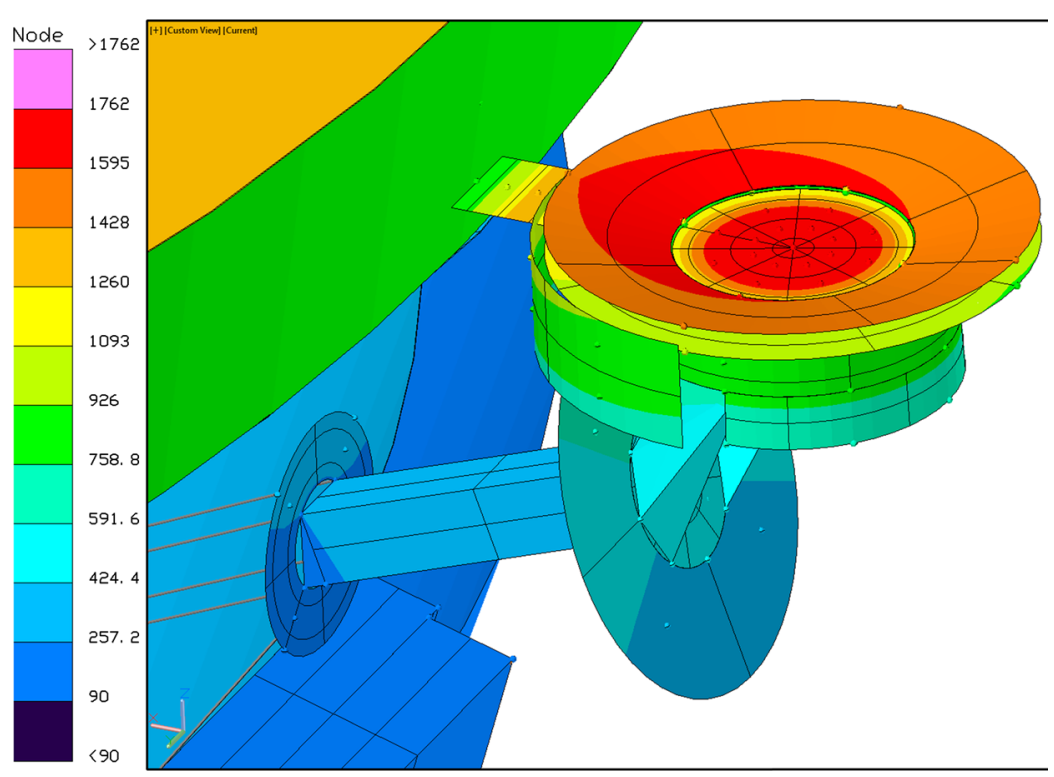

Fig. 21 Thermal model of SPC, showing predicted temperatures at close approach. Temperatures are in degrees Celsius

\subsection{SPC Thermal}

Four factors motivate keeping temperatures low within SPC: electron emission, mechanical integrity, signal amplification, and heat conduction to SPP. Hot conducting surfaces will generate thermionic emission that will be enhanced in the presence of strong electric fields by field-effect emission. These low energy electrons (several eV) can draw power from the HV supply by flowing to the modulator grid, and potentially interfere with the SPC solar wind signals. Thermionic emission from $\mathrm{W}$ materials peaks at about $1200{ }^{\circ} \mathrm{C}$ and is predicted to produce no more than $0.1 \mathrm{~mW}$ extra power draw on the SPC HV supply. Note that the accuracy of SPC flow angle and flux measurements are decoupled from any thermal or mechanical changes within the modulator assembly. Any change in the spacing between grids does not change the energy cutoff because they are maintained at a fixed voltage. Since the limiting aperture is always illuminated it is insensitive to any shifting of the entrance aperture.

Thermal simulations of SPC have been performed and results are shown in Fig. 21. During closest encounters the first grid in the FSU will reach above $1600{ }^{\circ} \mathrm{C}$ and the modulator housing walls will reach $1000^{\circ} \mathrm{C}$. Though still hot, the components at the anti-sunward side of the cup are relatively cooler; the collector plates will be about $700{ }^{\circ} \mathrm{C}$ and the back of the collector housing will be approximately $600^{\circ} \mathrm{C}$. This is well below the temperature range the materials and components have been tested to.

The thermal model was compared to measurements taken under realistic illumination conditions with the phase B prototype in the Solar Environment Simulator (SES), which is described in detail in Cheimets et al. (2013). The SES uses four short-arc Xenon lamps with broad-spectrum reflectors, water cooled mirrors and focusing optics, and a water-cooled window into a vacuum chamber to deliver precisely calibrated, stable light that mimics the intensity, spectrum, and angular spread of sunlight at different phases in the SPP mission. 


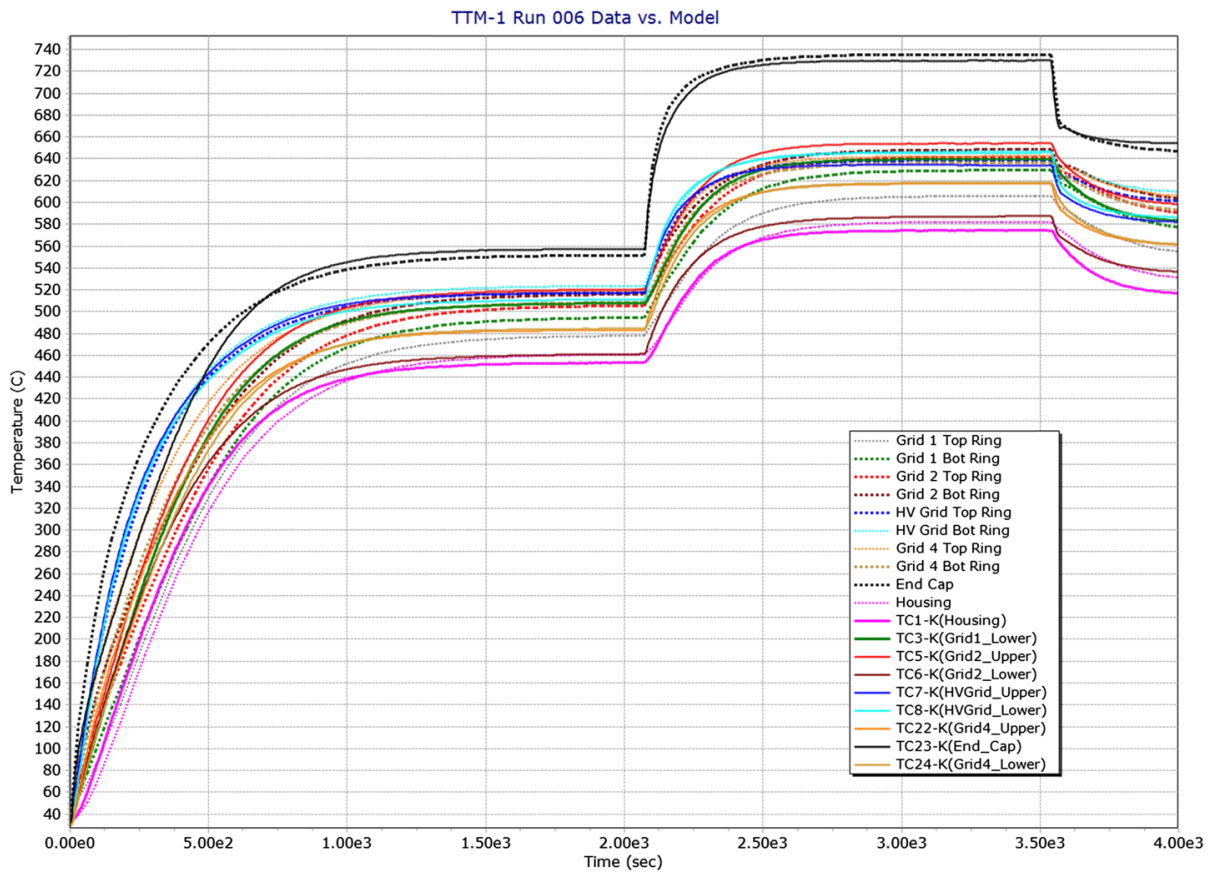

Fig. 22 Thermal model predictions (dashed lines) compared to measurements in the SES (solid lines) with the phase B prototype as a function of time. In this 1.1 hour experiment SPC was exposed to three different light levels, with time allowed for the instrument to come into thermal equilibrium at the new level (hundreds of seconds) and for instrument performance characterization measurements to be taken (thousands of seconds)

A scanning optical thermopile with NIST-traceable calibration is used to calibrate and control the photon flux of the SES onto SPC, allowing us to precisely validate the optical and thermal performance of the instrument against a known photon input. A comparison of the thermal model and measurements from thermocouples distributed throughout the instrument prototype is shown in Fig. 22. In this particular exposure three different illumination cases were presented to the instrument, with the temperatures allowed to come to equilibrium before moving to the next case. One can clearly see that not only is the model a good predictor of the equilibrium temperatures, it also performs well during the transition periods, providing further confidence that the assumptions in the thermal model are accurately reflecting the instrument. In addition to validating the instrument thermal design and performance, the SES has also been used to demonstrate that the instrument can survive the extreme thermal gradients and mechanical strains included by the photon-induced heating, to confirm predictions of thermoionic and photoelectric emission, to verify that the instrument high voltage performance as expected over temperature, and to confirm the energy resolution and measurement noise are uneffected by changes in light level and temperature.

\subsection{SPC Calibration Plans}

Test and calibration of SPC will be performed at two separate locations. A high-fidelity ion beam at MSFC will be used for calibration and characterization of the measurement 
Fig. 23 SPC current

measurements with an ion beam (signal current) and with no beam (noise current) as a function of collector assembly temperature mapped to effective distance from the Sun, indicating that the instrument noise level is insensitive to temperature

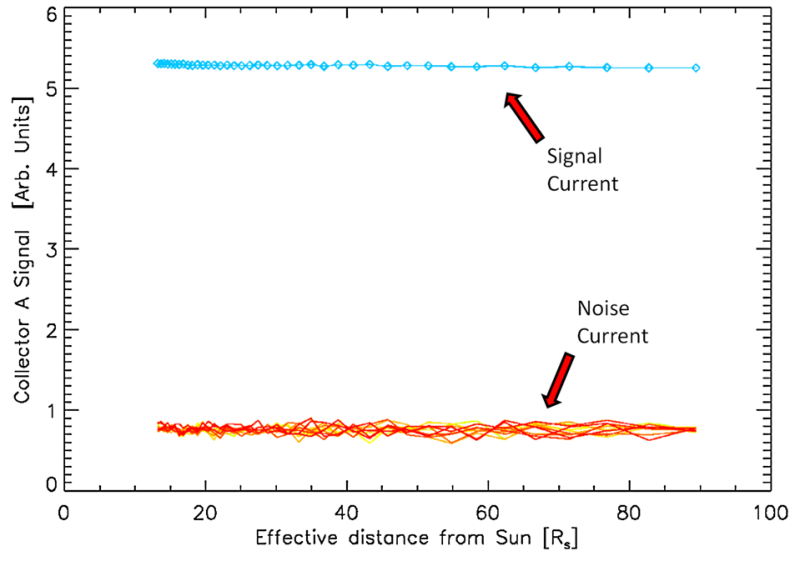

performance of the instrument at room temperature. This beam is capable of providing simultaneous ion and e-beams over the entire range of energies and fluxes. Instruments within SWF perform beam diagnostics and accurate monitoring of beam current and energy and spatial profiles. SWF was previously used to demonstrate performance of SPC prototypes. SPC test and calibration on the ground consists of verification of design and absolute determination of instrument response. SWF is first used to verify the transparency of the grids within SPC and the angular response of the instrument by rotating the instrument while exposed to a steady beam. Since these characteristics can be derived analytically this test simply verifies the design of the instrument. The energy response of SPC is verified by systematically sweeping ion and e-beams in energy and recording the resulting currents on the collector plates. As Fig. 18 demonstrated, the center energy and the width of the cutoff are easily determined.

Absolute calibration of SPC follows standard FC calibration procedures used by the SWEAP team on previous investigations. First, the relative calibration of the four collectors is determined by exposing each collector plate to the same beam current. One of the collectors is then disconnected from the instrument electronics and connected to a precision electrometer. The absolute calibration is then determined by comparing the current recorded by the electrometer with the signals returned by the other three collectors. The measured response generally agrees within a percent with theoretical predictions.

Early testing of the SPC prototype and electronics demonstrated that the instrument response to a given ion or electron current is not a function of the temperature of the electronics, and that temperature dependent changes in electronics noise levels were below the instrument sensitivity requirement. The phase B prototype was fitted with heaters to raise the temperature of the collector plates and collector housing, while the instrument was exposed to a proton beam that was steady in energy and flux. Figure 23 shows the response of the instrument to this beam as the temperature was raised from room temperature up to close-approach levels $\left(450^{\circ} \mathrm{C}\right)$. The figure converts that temperature into the corresponding distance of SPP from the Sun assuming temperature roughly scales inversely with the square root of distance from the Sun from radiative arguments (since there is no detectable change the accuracy of this mapping to distance is not particularly important).

The FEU may have some temperature dependence that will be characterized during the development of the instrument. A heater will be used to vary the pre-amplifier temperatures over their expected ranges and measure any dependence. For comparison, the temperature dependence of the entire electronics processing system for the Wind FC is linear and less 
than $0.01 \%$ per ${ }^{\circ} \mathrm{C}$, suggesting a maximum variation of a few percent over the possible temperatures of SPC electronics that could then be corrected in the processing pipeline.

As with other FCs, SPC will have an internal calibration mechanism to permit aliveness testing on the ground during integration and environmental testing and after launch to perform long-term trending of instrument response. The calibration currents are formed by a voltage divider network which divides a precision voltage oscillating at the modulator driver frequency. These measurements are used to determine the stability of the sensor properties, to identify temperature dependencies of the instrument response, and to provide a pre-flight baseline for in-flight calibration of the instrument. The availability of this internal calibration system also reduces risk throughout spacecraft integration and testing. The response of the internal system changes slightly if the signal cables are disconnected, allowing SWEAP to monitor instrument connections during spacecraft I\&T without the use of a particle beam.

The SPC will be integrated into the Suite for initial testing. The integrated SWEAP Suite will then be integrated into the SPP spacecraft.

\section{The Solar Probe ANalyzers (SPAN)}

\subsection{SPAN Overview}

SWEAP has three electrostatic analyzers (ESAs) to measure the three dimensional velocity distribution functions of ions and electrons with high angular, temporal, and energy resolution. The

SPAN-A module has two electrostatic analyzers (ESAs) to measure ions and electrons from the ram direction and nadir, and SPAN-B consists of a single ESA to measure electrons from the anti-ram direction. SPAN-A is located on the ram-direction side of SPP and SPAN-B is on the anti-ram side. Significant savings in mass are realized by combining the electron and ion ESAs (a lesson learned from FAST and THEMIS). Electrostatic deflectors extend the narrow planar intrinsic angular FOV of each ESA to $240^{\circ} \times 120^{\circ}$. Together the SPAN electron sensors provide a nearly $4 \pi \mathrm{sr}$ FOV for electrons (excluding the region of the sky blocked by the heat shield). Meanwhile, SPAN-A and SPC provide a continuous view of the solar wind ions, with SPAN-A providing the primary measurement at closest approach, when the velocity aberration from the lateral motion of the spacecraft brings the solar wind into the field of view. SPAN-A includes a pre-acceleration stage and carbon foils, closely based on the MAVEN STATIC design (McFadden et al. 2015), allowing the separation of solar wind protons, alpha particles, and heavier species. All three sensors include both mechanical and electrostatic attenuators that provide a broad dynamic range, allowing optimal sensitivity over the entire SPP orbit.

The SPAN design results from a long and successful history of ESA development at the U.C. Berkeley Space Sciences Laboratory, including sounding rockets, Mars Global Surveyor ER (Mitchell et al. 2001), Wind 3D-Plasma (Lin et al. 1995), Lunar Prospector, FAST Plasma Experiment (Carlson et al. 1998), THEMIS (McFadden et al. 2008), and MAVEN (Halekas et al. 2013; McFadden et al. 2015). The UCB group also participated in the development of the STEREO IMPACT SWEA instrument (Sauvaud et al. 2007). The SPAN sensors closely follow heritage designs from MAVEN, which add deflectors to the proven heritage analyzer to provide a large out-of-plane. The electronics use heritage designs developed for MAVEN, with the addition of some ASIC electronics to reduce mass and power.

The SPAN sensors utilize the classic top-hat hemispherical ESA design developed by UCB (Carlson et al. 1983) that affords a uniform response over a planar $360^{\circ} \mathrm{FOV}$. Particles 
entering the analyzer are selected for energy per charge (E/q) by a voltage applied to the inner hemisphere. This voltage is swept from near zero to several $\mathrm{kV}$ to measure ion and electron energies as low as a few eV/q or as high as $30 \mathrm{keV} / \mathrm{q}$, providing excellent energy coverage and resolution. Angular resolution is provided in one plane by discrete segmented anodes and out of that plane by electrostatic deflectors, resulting in a broad instrumental field of view appropriate for a non-spinning spacecraft like SPP. Similar design challenges have been met by UCB on STEREO and MAVEN, both 3-axis stabilized spacecraft like SPP.

Both ion and electron sensors use microchannel plate (MCP) detectors for particle detection, and discrete anodes for MCP charge collection. The electron sensor uses chevron-pair detectors, while the ion sensor utilizes a Z-stack to provide larger pulses for constant fraction discrimination timing measurements of start and stop pulses. Particle events from each anode are then accumulated in counters and read-out each 0.5 milliseconds. Pulse-counting electronics for the electron sensors utilize a multi-channel preamplifier ASIC developed by LPP for the Solar Orbiter mission. The ion sensor also utilizes ASIC electronics for constant fraction discrimination and time-to-digital conversion, for the purposes of measuring time-of-flight and thus determining ion mass per charge. Low voltage and high voltage power supplies used designs closely based on FAST, THEMIS, and MAVEN. The sensors also include a one-time use, manually resettable Shape Memory Alloy (SMA) aperture release mechanism that keeps the sensor sealed until after launch, preventing contamination of the MCP detectors. This mechanism also acts as a pressure relief valve, allowing nitrogen purge to be applied periodically rather than continuously, to simplify integration and testing. The one-time cover release mechanism is tightly integrated with the mechanical attenuator, which is also based on SMA technology.

SPAN-B measures electrons only and is a near duplicate of the SPAN-A e-analyzer (the anode patterns are different), with an orthogonal orientation on the opposite side of the spacecraft. The ability of SWEAP to use the same design for both SPAN-A and SPAN-B electron sensors provides significant savings in design time and analysis.

We relied on detailed charged particle optics simulations to optimize our basic heritage electrostatic analyzer design and construct instruments optimized for the Solar Probe Plus mission design. Sample optics simulations are shown below in Fig. 24, for a given deflection direction (determined by the ratio of a voltage applied to one of the two deflectors with respect to the inner hemisphere voltage) based on software described previously (McFadden and Carlson 1998). All three sensors utilize an identical optics design for the electrostatic analyzer and deflectors, with the SPAN-A ion sensor adding a time-of-flight section below the analyzer. The science goals of SWEAP require very high energy and angular resolution, so the gap between hemispheres is chosen to be rather small, $\Delta R / R=0.03$. Without careful mechanical design, such small gaps can result in analyzer concentricity problems, producing a non-uniform sensor response; we employ an ESA design strategy to minimize machining tolerance impact on concentricity as discussed and demonstrated by McFadden et al. (2008), resulting in extremely precise concentricity and more uniform throughput around the output of the ESA (McFadden and Carlson 1998). The electrostatic deflectors above and below the main sensor aperture can redirect the main cylindrically symmetric planar FOV out of plane between $\pm 60^{\circ}$ in elevation, providing a possible $360^{\circ} \times 120^{\circ} \mathrm{FOV}$ for a sensor. For Solar Probe Plus, blockage by the spacecraft reduces this FOV to approximately $240^{\circ} \times$ $120^{\circ}$ for all three sensors. Deflection does change the angular response of the sensor, due to focusing/de-focusing effects, but as shown in Fig. 25, the angular resolution of the sensors remains very good (better than $\sim 10^{\circ}$ ) over the entire deflection range. In addition, the geometric factor is constant over the entire effective field of view, only rolling off at deflection 
Fig. 24 Top: SPAN optics simulation, showing several ray tracing trajectories (in red) for a single deflection voltage.

Particles enter from the left, pass between the deflectors, travel through the curved plate analyzer, and strike the MCP at the exit of the curved plate. Bottom:

Normalized geometric factor of the optics as a function of voltage on the spoiler as a fraction of the voltage on the inner curved plate
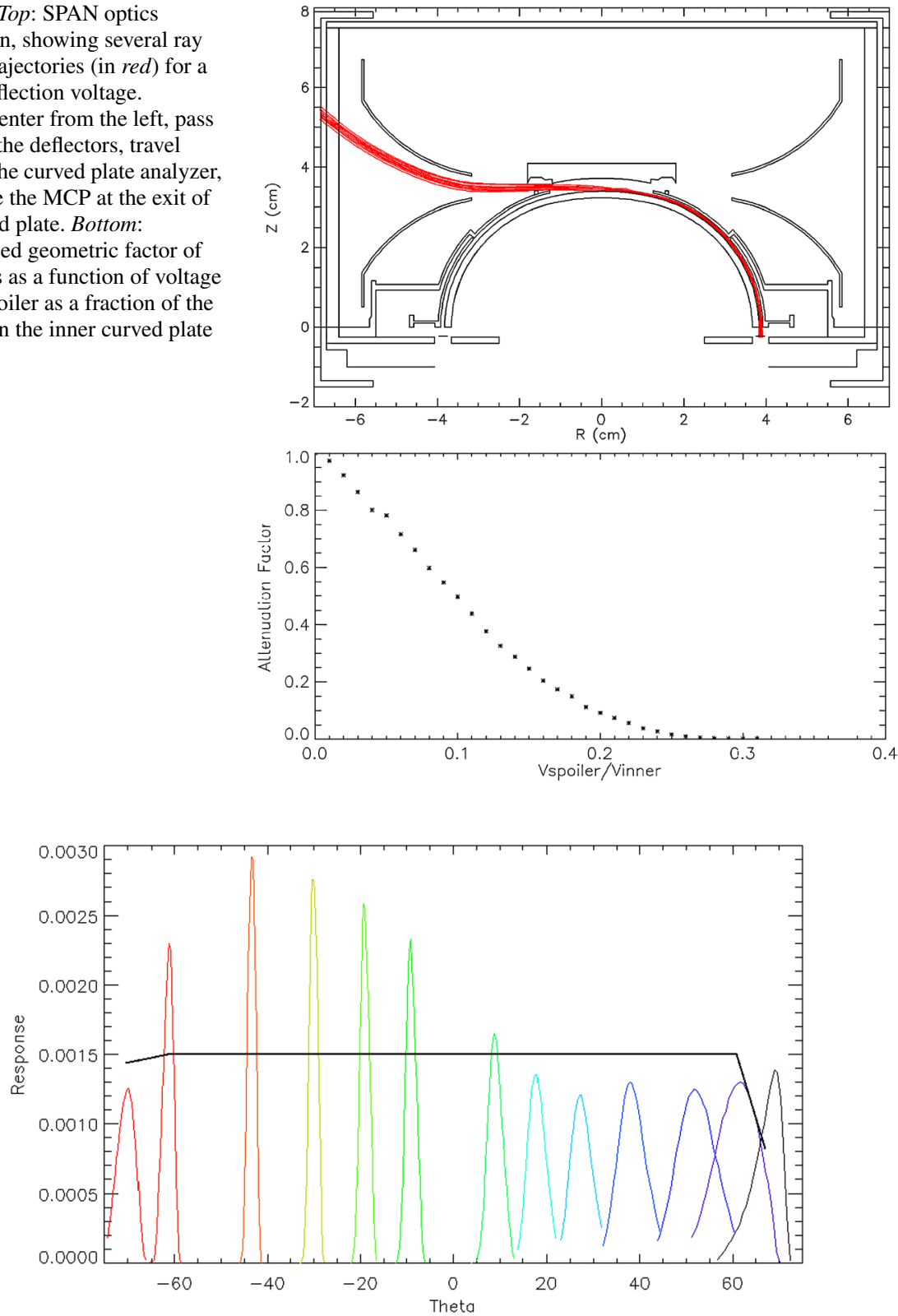

Fig. 25 Deflection angle resolution for selected deflection steps. Each color represents the response of the instrument in arbitrary units as a function of angle for a given deflection setting. The total instrument geometric factor is shown in black

angles higher than $\pm 60^{\circ}$. The energy/charge resolution also remains constant at $\sim 7 \%$ over this entire range.

Due to the large dynamic range in particle fluxes encountered between perihelion (0.046 AU) and aphelion (0.7 AU), and the desire to measure extreme events, each sensor 
will include a mechanical attenuator that can be engaged to prevent detector saturation. The attenuator design is a modification of the bi-stable nano-muscle SMA designs successfully used for the STEREO STE, THEMIS SST, and MAVEN SWIA and STATIC instruments. The attenuator introduces a "visor" with a small slit at the opening to the curved plate section that reduces the sensitivity by a factor of approximately 10. This attenuator has the additional benefit of slightly improving the intrinsic energy/charge and angular resolution of the instrument.

In addition, the SPAN sensors will incorporate electrostatic attenuators capable of reducing the sensitivity of the analyzers by an additional factor of $\sim 10$. We accomplish this attenuation by splitting the outer hemisphere (normally at ground), and placing a voltage (up to approximately $25 \%$ of the inner hemisphere voltage) on the lower section of the outer hemisphere (the "spoiler") proportional to the voltage on the inner hemisphere. This in effect produces two half-analyzers with different electric fields between them, and correspondingly different energy transmissions, thereby reducing the over-all band-pass of the instrument. In contrast to the mechanical attenuation, the electrostatic attenuator can be switched on and off very quickly, even within a single energy sweep, allowing measurement of minority constituents with full sensitivity while "spoiling" the high flux of protons, assuming that the two species are at different energy/charge.

One could imagine using a single attenuator with an attenuation factor of 100 , rather than two separate attenuators with smaller attenuation factors. However, given the desire to make extremely fast measurements on Solar Probe Plus, a larger attenuation factor would result in an unacceptably large step in counts per accumulation. We achieve far better science measurements by utilizing two attenuation stages, providing smoothly varying dynamic range. In addition, this combination allows nearly instantaneous response and optimization of the geometric factor to rapidly varying fluxes, since the combination of the mechanical and electrostatic attenuators allow for a two-step adjustment-one order of magnitude that happens infrequently (mechanical) and an additional order of magnitude that can be adjusted rapidly as needed (electrostatic).

The HV supply that controls the inner hemisphere voltage and the deflector plate voltages is limited to $3-4 \mathrm{kV}$. This provides ample headroom to measure particles up to $30 \mathrm{keV}$; however, full 60-degree deflection is only possible up to about $4.5-6.0 \mathrm{keV}$, depending on the details of the final high voltage design. Particles with higher energies are measured within a reduced angle range. This is a fundamental limitation of any instrument that relies on a similar electrostatic deflection system, and forces careful consideration of instrument mounting and operation. A significant benefit of our chosen orientation for the SPAN-A module is the ability to attain the highest energies in the near nadir direction without need for deflection. Any system that relies purely on electrostatic deflection to measure particles near nadir will necessarily fail at high energies; our accommodation for SPAN-A overcomes this issue, and guarantees measurements of solar ions over the full energy range.

\subsection{SPAN Estimated Performance}

In the plane of the instrument aperture, the intrinsic azimuthal resolution of the SPAN sensors is very high because of the focusing properties of the top-hat electrostatic analyzer, but the actual azimuthal angular resolution is determined by the size of the discrete anodes. In order to minimize power required from the discrete preamplifiers, angular resolution over the $240^{\circ}$ FOV of both the SPAN-A and SPAN-B electron sensors is divided into eight high resolution $6^{\circ}$ anodes, and eight lower resolution $24^{\circ}$ anodes. The high-resolution anodes are chosen to image near the sun in order to capture the electron strahl. The SPAN-A and SPANB electron sensor FOVs are oriented to optimize their combined FOV as shown in Fig. 13 

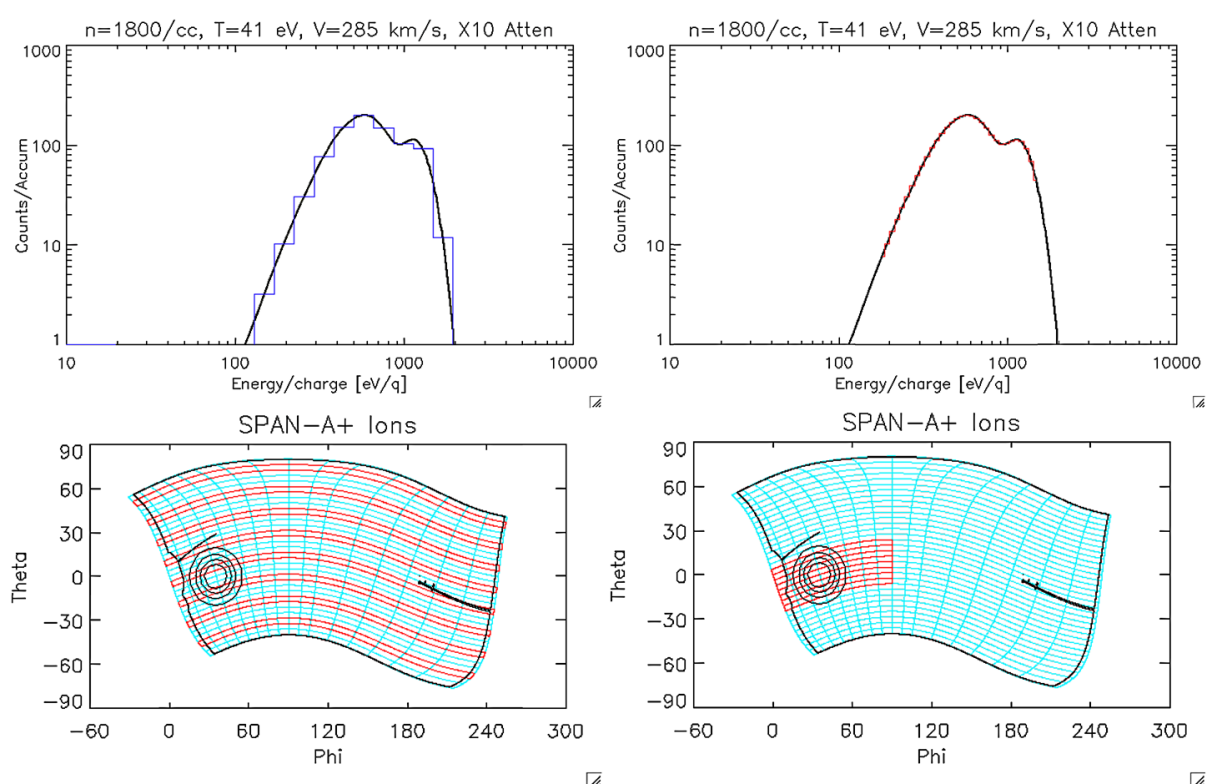

Fig. 26 Proton energy spectra and angular distributions demonstrating the expected measurement resolution for coarse (left) and targeted (right) sweeps, for a two-component solar wind proton distribution at closest approach

in Sect. 2. This orientation, with the SPAN-B FOV rotated $90^{\circ}$ relative to the SPAN-A FOV, provides optimal coverage of electrons up to $4.5 \mathrm{keV}$ over the whole sky. The finely segmented anodes are placed optimally to measure the strahl, and the alignment of the electron FOVs ensures full energy coverage towards the Sun.

The anodes for the SPAN-A ion sensor, meanwhile, are chosen to have high resolution in the directions the center of the ion core will flow from, and lower resolution elsewhere. The $247.5^{\circ} \mathrm{FOV}$ is divided into $1011.25^{\circ}$ anodes in the near-Sun direction, and $622.5^{\circ}$ anodes across the remaining FOV further from the Sun. The ion sensor is mechanically combined with the electron sensor on the RAM side of the spacecraft since they share a common FOV.

As described in more detail below in Sect. 5.1, all three SPAN sensors typically operate in "Alternating Sweep" mode, interleaving low resolution measurements of the entire available phase space (sparsely sampled) with full resolution targeted measurements of selected portions of the distribution (fully sampled). We show an example measurement of the solar wind protons below in Fig. 26 in order to demonstrate SPAN-A's ability to cover all of phase space, while still resolving the peak, at high cadence (both distributions can be obtained within less than half a second). The alpha particles, also present in this energy range, are separated using the mass-resolving capability of SPAN-A, and not shown in this figure. With the expected mass/charge resolving capability of SPAN-A, we will be able to generate fully distinct VDFs for protons and alphas.

\subsection{SPAN Electrical}

Primary power for each SPAN sensor is provided by the SWEM. Each sensor has a low voltage power converter that receives regulated $22 \mathrm{~V}$ from the SWEM and converts it to provide analog and digital secondary voltages isolated from the primary service. These voltages 
are used to operate a digital board, high voltage power supplies, and front end electronics. A backplane also provides interconnects between the individual electronics boards, some of which are also connected directly by stacking connectors.

The digital board for each sensor is focused around an FPGA. Lookup tables that define the high voltage sweep and the product accumulation are stored in local memory on the board. Utilizing these lookup tables, the FPGA accumulates counts from the front-end electronics and bins them into configurable data products, as well as controlling the high voltage sweep (via DAC outputs) that defines what energies and angles are sampled at each time step, as well as the electrostatic attenuation of the spoiler. The FPGA also controls an ADC to read engineering data, collects and packetizes housekeeping information, and controls the one-time cover and mechanical attenuator power. Finally, the sensor FPGA interfaces with the SWEM, receiving commands and a clock and sending telemetry as controlled by SWEM command and product lookup tables.

The high voltage power supplies provide fixed (adjustable) bias voltages for the microchannel plate detectors and sweeping (lookup table-driven) voltages for the inner hemisphere, electrostatic deflectors and spoiler electrostatic attenuator. For the ions, an additional $15 \mathrm{kV}$ supply provides a pre-acceleration voltage for the time of flight section. The sweeping supplies utilize optocoupler circuitry for the outputs. The high voltage supplies utilize the primary $22 \mathrm{~V}$ from the SWEM, switched through the digital board, and controlled by DAC outputs from the digital board. For the electrons, all high voltage power supplies share one board, while for the ions the pre-acceleration and MCP supply are housed on a separate board from the sweep supplies.

The front end for the electron sensors consists of a single board which provides a mounting point for the chevron microchannel plates (MCPs), collects charge pulses from the back face of the MCPs on a segmented anode, and utilizes a 16-channel preamplifier ASIC to produce digital pulses suitable for accumulation in the digital board.

The front end for the ion sensors consists of an anode board that provides a mounting point for the Z-stack microchannel plates and collects output pulses on segmented start and stop anodes. Each anode is capacitively coupled to an ASIC-based constant fraction discriminator providing a digital output pulse. These pulses are fed into a time-to-digital conversion utilizing ASIC parts, located on the digital board.

\subsection{SPAN Mechanical}

The mechanical design of SPAN-A and SPAN-B as of Mission PDR is summarized in Fig. 27 (SPAN-B) and Fig. 28 (SPAN-A). Since the electron ESA in SPAN-A is essentially identical to the electron ESA in SPAN-B, we begin with a review of SPAN-B.

Starting from the left side of Fig. 27, the top of SPAN-B consists of a deployable onetime cover (re-closeable on the ground) that protects the ESA from contamination before launch. Immediately below the cover is a mechanical attenuator that is used to regulate the geometric factor of the sensor. The main optics section begins with a broad entrance aperture surrounding a pair of electrostatic deflectors that steer the instantaneous field up and down. After passing through the deflectors, particles travel between a pair of hemispheres, which have a voltage between them that only allows particles in a narrow band of energy to reach the exit. By placing a small voltage on the bottom half of the outer hemisphere ("the spoiler") we can reduce the sensitivity of the analyzer by an order of magnitude, essentially be de-tuning the response of the analyzer. Particles that successfully traverse the electrostatic optics impact a chevron pair of microchannel plates with a high voltage bias across them, which produce a secondary electron cascade with a multiplication factor of a few million, producing a pulse with measurable amplitude that is collected on a segmented anode. 
Fig. 27 Cross section of the SPAN-B instrument

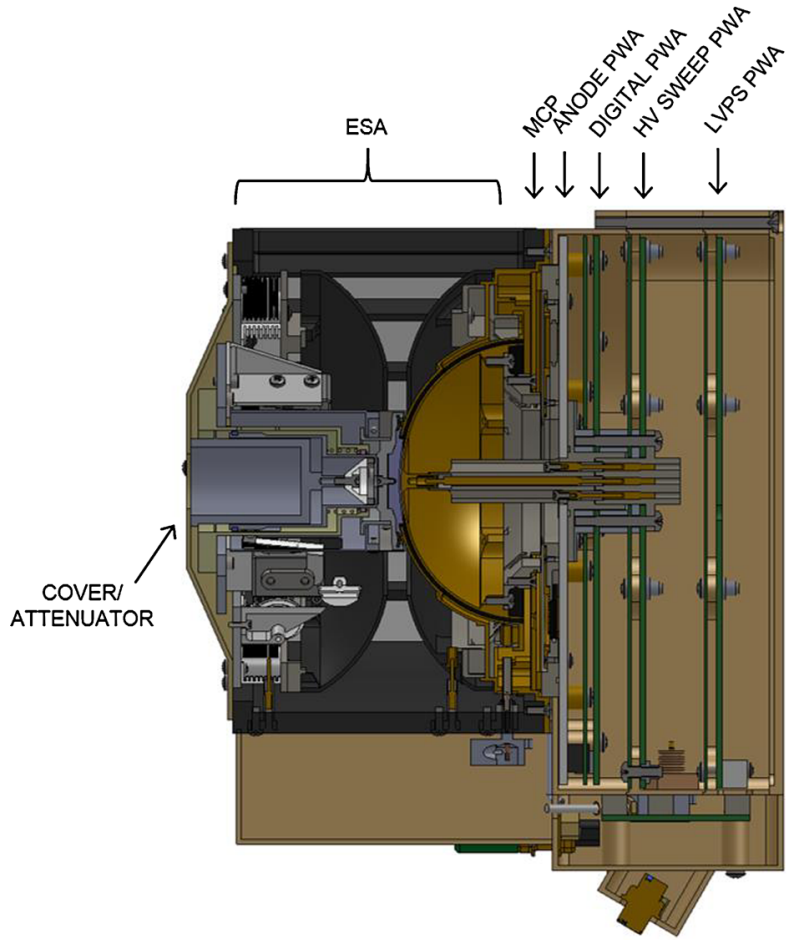

A central tower penetrates the anode and digital boards, bringing high voltage to the inner hemisphere from the high voltage power supply. The anode board is directly mounted in a transition plate which provides a smooth join between the analyzer and the electronics box, while the succeeding electronics cards (digital, high voltage power supply, and low voltage power converter) are mounted in frames which stack together, with a back plane mounted perpendicularly and providing signal and power routing between electronics boards.

SPAN-A is shown in cross section in Fig. 28. The SPAN-A electron sensor has an identical mechanical design to SPAN-B, but is mounted together with the SPAN-A ion sensor. The ion sensor also has a nearly identical design, including the same analyzer optics, but with a few critical differences. Instead of an anode board directly at the exit of the analyzer, the SPAN-A ion sensor has a time of flight sensor. A pre-acceleration voltage accelerates ions to a high enough energy to reliably penetrate a carbon foil, producing start electrons that are then guided to the inner half of the microchannel plates. The ion then continues to a second carbon foil, generating a stop pulse. The stop electrons have sufficient energy to penetrate a thick foil and generate a stop pulse, while the primary ions stop in the thick foil, eliminating a primary background source for ion mass composition sensors. The remainder of the electronics box has a similar design, albeit with more boards than the electron sensors in order to accommodate the additional high voltage power supply for the pre-acceleration.

\subsection{SPAN Thermal}

Since the SPAN instruments are in shadow during scientific operations close to the Sun, the thermal design of these sensors is much more straightforward than for SPC. However, this does not mean that it is completely straightforward. Indeed, the SPAN sensors must contend 


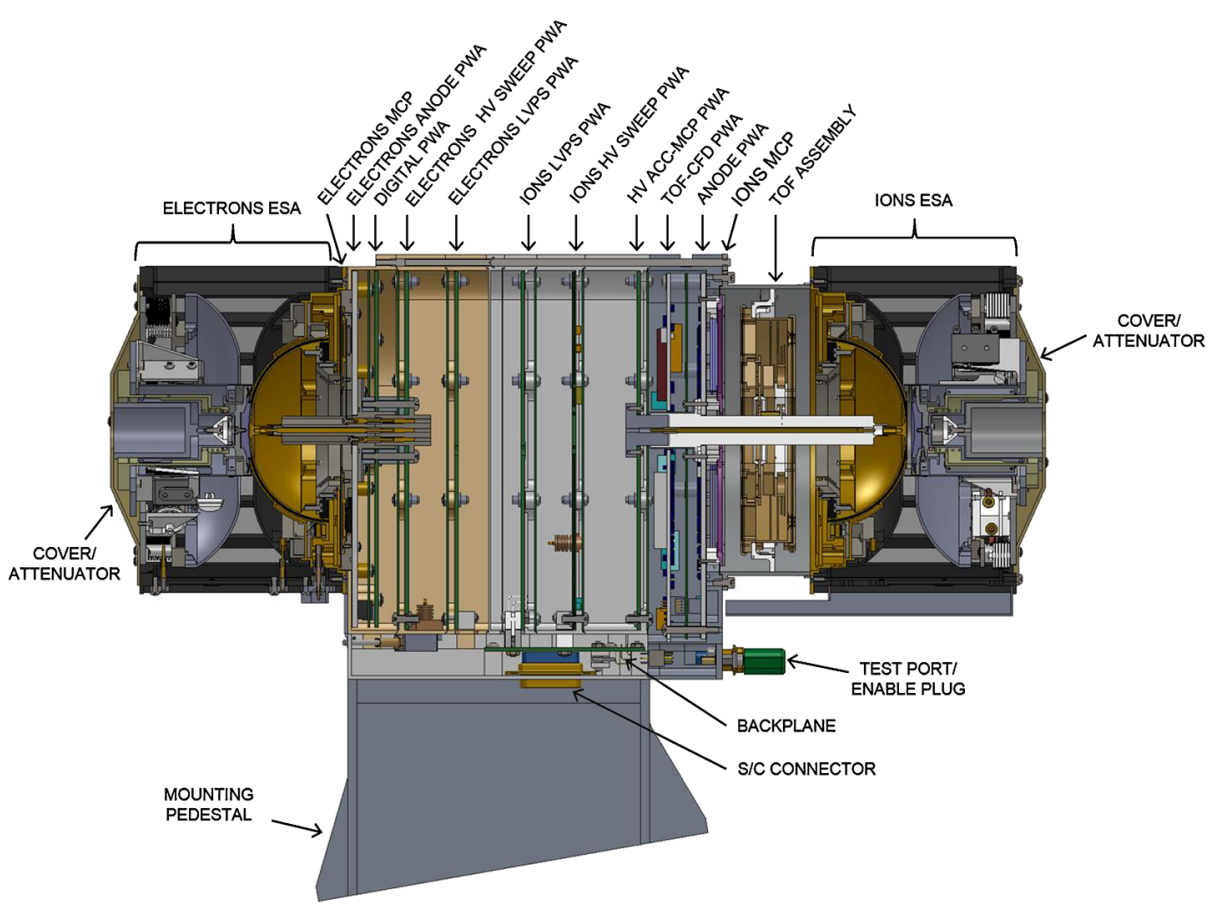

Fig. 28 Cross section of the SPAN-A+ instrument. In this orientation the electron instrument is on the left and the ion instrument is on the right

with a somewhat non-intuitive thermal problem. Far from the Sun, the spacecraft must be able to rotate in order to orient the high gain antenna on the anti-ram side of the spacecraft toward Earth for communications. This results in prolonged illumination of the SPAN-B sensor. The level of heat absorbed by SPAN-B must be limited in order to prevent damage to the deployment and attenuator mechanisms in the electron ESA sensor. Unfortunately treatment of SPAN-B surfaces to reduce absorption of heat far from the Sun when the spacecraft is rotated and the anti-ram side is illuminated results in very low operating temperatures for SPAN-B when it is close to the Sun. Ironically, at closest approach, while the Sun-facing instruments and spacecraft components struggle with extreme high temperatures, SPAN-B risks falling below its minimum operating temperature. As a result, an operational heater was added to SPAN-B.

\subsection{SPAN Calibration Plans}

UCB will perform subsystem-level testing, and instrument-level vibration and thermal vacuum tests prior to suite-level integration at SAO. Calibration is done at UCB using existing high-vacuum facilities. These calibration procedures have been developed and optimized by UCB scientists over decades (McFadden et al. 2008), and result in extremely well calibrated and characterized instrumental response, as most recently demonstrated for MAVEN (Halekas et al. 2013; McFadden et al. 2015).

Ground testing of SPAN includes standard functional tests and subsystem optimization prior to assembly and calibration. Pulse-counting electronics are tested for response, including threshold, output pulse length, and dead time to assure nearly identical characteristics. 
Opto-coupler HV sweep electronics are tuned to provide near zero offsets, to provide nearly identical (better than $1 \%$ ) high voltage control gain, and to have rapid settling (1-2 ms) with no overshoot. MCP detectors are matched for current and selected for low background rates and gain uniformity. After sensor assembly, calibrations are performed in high vacuum, including tests for background noise sensitivity, MCP background rates, MCP pulse height distribution, energy-angle response, concentricity tests, relative anode sensitivity characterization, uniform angular response, and sweep mode performance.

The sensors include test-pulsers that allow complete tests of the LV electronics outside of high vacuum. Since high voltage and MCP detectors can only be operated in high vacuum, this allows modular and parallel testing of different components of the instrument, achieving the most efficient use of resources and time, and resulting in the best instrument performance and calibration.

In-flight calibrations will be performed within SWEAP and with other sensors on the spacecraft. For example plasma frequency measurements can be used to check absolute calibration, and e-and ion bulk moments can be compared to check both absolute and relative calibrations. Use of the preamplifiers with a programmable threshold allows monitoring of the MCP gain to allow optimization of detector operations. HV supplies are completely programmable and designed to have adequate range to compensate for MCP degradation over an extended mission. Relative anode efficiency calibrations can be performed in flight, and any energy-dependent efficiency corrections can be determined by cross-calibration of sensors.

\section{Science Operations, Data Processing \& Data Products}

The SWEAP instrument suite operates autonomously during each solar encounter, with the SWEM controlling each of the SWEAP sensors, formatting data products, archiving high resolution data to internal storage in the SWEM, and transmitting summary data to the spacecraft for quick transmission to Earth following each encounter. As of PDR, the SWEAP Suite was allocated 20 Gbits of data volume per orbit to meet the Level 1 science goals described in Sect. 1.1. This section describes the individual operating modes of the sensors, the SWEAP science operations plan, and the data products that will be produced and distributed to the public.

\subsection{Software/Instrument Modes}

The sensor modes are determined by a command plan timeline that is uploaded to the SWEM from the SWEAP SOC. The sensors operate autonomously under direction of the SWEM. The available science modes are described for the SPC and the SPANs.

SPC always operates at the same cadence, returning the four currents (4I) for a given E/q window (E) 128 times every 0.874 seconds. The SWEM decides what data products to form with SPC by commanding the instrument to scan through different values of E. In Proton Tracking (PT) mode, SPC scans across the center of the proton VDF with $8 \mathrm{E}_{||} / \mathrm{q}(8 \mathrm{E})$ windows, producing $8 \mathrm{Ex} 4 \mathrm{I}$ measurements 16 times every 0.874 seconds. These measurements are easily solved for $\mathbf{V}_{\mathrm{p}}, \mathrm{T}_{\mathrm{p}}$, and $\mathrm{n}_{\mathrm{p}}$ of the core of the proton distribution. With the addition of magnetic field data on the ground, variation in $T_{p}$ with field direction can be used to solve for the temperature anisotropy of protons at a lower cadence. In Full Scan (FS) mode, SPC scans through 128E in 0.874 seconds over the full range of SPC voltages. A FS can be solved for alpha and proton core and beam bulk properties and for the e-strahl energy spectrum. In 
Flux Angle (FA) mode the SWEM increases the size of the window to at least $10 \%$ and places it either over the center of the proton VDF or in the e-strahl. Every measurement can then be solved for the flow angle and total flux at 128 times per 0.874 seconds. The FS and FA modes can be performed for electrons as well as ions.

The SPAN sensors operate in an almost entirely table-based fashion. Programmable lookup tables determine the voltages placed on the inner hemisphere, the two deflectors, and the electrostatic attenuator, at each sweep step (with an accumulation interval having a standard duration of approximately $0.874 \mathrm{msec}$ ). This allows tremendous flexibility in the instrument operation, allowing standard energy-angle sweeps where both energy and deflection angle are varied, or rapid 1-d or 2-d measurements where the instrument looks in only one or a few energies/directions, and measures at higher effective cadence.

The standard mode of both ion and electron sensors is termed "Alternating Sweep". In this mode, the instrument alternates between two kinds of energy/angle sweeps. The first sweep ("Coarse") covers all of phase space, but with coarse resolution and sparse sampling. Utilizing the count rates measured during this sweep, the second sweep ("Targeted") covers a limited region of phase space around the location of the peak, with the highest intrinsic resolution. The selection of the location of the targeted sweep based on the coarse sweep is completely configurable and also entirely table-based. These two sweeps together provide a full view of the particle distribution, together with a high-resolution view of the most interesting part (typically the core for the ions, and the strahl for the electrons). This can be achieved at a very high cadence, with each energy-angle sweep taking 256 accumulation intervals, or 0.224 seconds, and each product is thus obtained every 0.447 seconds.

The raw counts from these sweeps are binned in the instrument FPGAs in a table-based fashion. For the electrons, they will typically be binned into 3-d energy-angle histograms, and 1-d angle-averaged energy spectra. For the ions, given the additional dimension of mass, a number of different products will be produced, which average the four-dimensional measurements into products that preserve higher resolution in certain dimensions, while averaging over others.

Given the limitations on telemetry, combined with the extremely fast cadence of the measurements, we have no hope of returning all data to the ground. Therefore, we make substantial use of flash memory storage onboard. By having the capability to store data for an entire orbit, we can download a small fraction of the data at low time resolution as "Survey" products, then use human selection of the most interesting and scientifically valuable time periods to download selected "Archive" data at the highest time resolution. Nominal data products and survey/archive cadences are shown for each sensor in the tables below (Tables 5, 6, and 7), though given our table-based operation, we retain great flexibility to modify this as scientifically required.

The data products described for SPC and SPAN produce the measurements necessary to meet each of the SPP and SWEAP goals and objectives discussed in Sect. 1.1. On the ground, SPC and SPAN measurements are combined to produce a single continuous stream of bulk solar wind properties. In the first version of this product either SPC or SPAN measurements are selected based on the flow angle of the plasma. In the second version SPC and SPAN data are fitted simultaneously. The science data processing pipeline will determine the best fit solar wind parameters using the raw data from the two instruments, and find that this will greatly increase the value of the SWEAP measurements. Joint fits allow SPAN to make accurate temperature anisotropy determinations even when the center of the VDF is only seen by SPC. Joint fits also allow SWEAP to characterize subsonic or very low Mach number plasma, which might occur close to the Sun. A summary of the data products from the SWEAP sensors are presented in Table 8. 
Table 5 SPAN Electron Data (an additional 1.47 Gbits is allocated for non-encounter survey and/or higher archive return)

\begin{tabular}{|c|c|c|c|c|c|}
\hline Product & Description & $\begin{array}{l}\text { Encounter survey } \\
\text { cadence }\end{array}$ & $\begin{array}{l}\text { Archive } \\
\text { cadence }\end{array}$ & $\begin{array}{l}\text { Encounter survey } \\
\text { volume }\end{array}$ & $\begin{array}{l}\text { Archive } \\
\text { (assuming } 2 \% \text { return) }\end{array}$ \\
\hline SPAN-A-e Full 3d & $32 \mathrm{Ex} 8 \mathrm{dx} 16 \mathrm{~A}$ & $56 \mathrm{~s}$ & $0.437 \mathrm{~s}$ & 0.34 Gbits & 0.86 Gbits \\
\hline SPAN-B Full 3d & $32 \mathrm{Ex} 8 \mathrm{dx} 16 \mathrm{~A}$ & $56 \mathrm{~s}$ & $0.437 \mathrm{~s}$ & 0.34 Gbits & 0.86 Gbits \\
\hline $\begin{array}{l}\text { SPAN-A-e } \\
\text { Targeted 3d }\end{array}$ & $32 \mathrm{Ex} 8 \mathrm{dx} 8 \mathrm{~A}$ & $28 \mathrm{~s}$ & $0.437 \mathrm{~s}$ & 0.34 Gbits & 0.43 Gbits \\
\hline $\begin{array}{l}\text { SPAN-B Targeted } \\
3 \mathrm{~d}\end{array}$ & $32 \mathrm{Ex} 8 \mathrm{~d} \times 8 \mathrm{~A}$ & $28 \mathrm{~s}$ & $0.437 \mathrm{~s}$ & 0.34 Gbits & 0.43 Gbits \\
\hline $\begin{array}{l}\text { SPAN-A-e Energy } \\
\text { Spectra }\end{array}$ & $32 \mathrm{E}$ & $0.437 \mathrm{~s}$ & N/A & 0.34 Gbits & N/A \\
\hline $\begin{array}{l}\text { SPAN-B-e Energy } \\
\text { Spectra }\end{array}$ & $32 \mathrm{E}$ & $0.437 \mathrm{~s}$ & N/A & 0.34 Gbits & N/A \\
\hline Total encounter & & & & 2.04 Gbits & 2.58 Gbits \\
\hline
\end{tabular}

In this and subsequent tables the abbreviations $\mathrm{E}$ for energy, $\mathrm{d}$ for deflection, and $\mathrm{A}$ for anode are used in the description column

Table 6 SPAN Ion Data (an additional 1.33 Gbits is allocated for non-encounter survey and/or higher archive return)

\begin{tabular}{|c|c|c|c|c|c|}
\hline Product & Description & $\begin{array}{l}\text { Survey } \\
\text { cadence }\end{array}$ & $\begin{array}{l}\text { Archive } \\
\text { cadence }\end{array}$ & Survey volume & Archive ( $2 \%$ return) \\
\hline $\begin{array}{l}\text { Proton/Alpha } \\
\text { Full 3d }\end{array}$ & $32 \mathrm{Ex} 8 \mathrm{~d} \times 16 \mathrm{~A}$ & $56 \mathrm{~s}$ & $3.5 \mathrm{~s}$ & 0.68 Gbits & 0.22 Gbits \\
\hline $\begin{array}{l}\text { Proton/Alpha } \\
\text { Targeted 3d }\end{array}$ & $32 \mathrm{Ex} 8 \mathrm{dx} 8 \mathrm{~A}$ & $56 \mathrm{~s}$ & $0.437 \mathrm{~s}$ & 0.34 Gbits & 0.86 Gbits \\
\hline Heavy Full 4d & $32 \mathrm{Ex} 8 \mathrm{dx} 16 \mathrm{Ax} 8 \mathrm{M}$ & $224 \mathrm{~s}$ & $3.5 \mathrm{~s}$ & 0.67 Gbits & 0.86 Gbits \\
\hline $\begin{array}{l}\text { Heavy Full } \\
\text { Halo-E }\end{array}$ & $32 \mathrm{Ex} 64 \mathrm{M}$ & $112 \mathrm{~s}$ & $0.874 \mathrm{~s}$ & 0.08 Gbits & 0.22 Gbits \\
\hline $\begin{array}{l}\text { Heavy Full } \\
\text { Halo-Angle }\end{array}$ & $4 \mathrm{Ex} 8 \mathrm{dx} 16 \mathrm{Ax} 32 \mathrm{M}$ & $112 \mathrm{~s}$ & $3.5 \mathrm{~s}$ & 0.67 Gbits & 0.43 Gbits \\
\hline $\begin{array}{l}\text { Heavy Targeted } \\
4 \mathrm{~d}\end{array}$ & $32 \mathrm{Ex} 8 \mathrm{~d} \times 8 \mathrm{Ax} 8 \mathrm{M}$ & $447 \mathrm{~s}$ & $3.5 \mathrm{~s}$ & 0.17 Gbits & 0.43 Gbits \\
\hline $\begin{array}{l}\text { Heavy Targeted } \\
\text { Mass-E }\end{array}$ & $32 \mathrm{Ex} 64 \mathrm{M}$ & $112 \mathrm{~s}$ & $0.874 \mathrm{~s}$ & 0.08 Gbits & 0.22 Gbits \\
\hline $\begin{array}{l}\text { Heavy Targeted } \\
\text { Mass-Angle }\end{array}$ & $4 \mathrm{Ex} 8 \mathrm{~d} \times 8 \mathrm{Ax} 32 \mathrm{M}$ & $224 \mathrm{~s}$ & $3.5 \mathrm{~s}$ & 0.17 Gbits & 0.22 Gbits \\
\hline $\begin{array}{l}\text { Proton/Alpha } \\
\text { Full Spectra }\end{array}$ & $32 \mathrm{E}$ & $14 \mathrm{~s}$ & $0.437 \mathrm{~s}$ & 0.02 Gbits & 0.14 Gbits \\
\hline $\begin{array}{l}\text { Proton/Alpha } \\
\text { Targeted Spectra }\end{array}$ & $32 \mathrm{E}$ & $0.437 \mathrm{~s}$ & N/A & 0.68 Gbits & N/A \\
\hline $\begin{array}{l}\text { Proton/Alpha } \\
\text { Moments }\end{array}$ & $13(\mathrm{n}, \mathrm{v}, \mathrm{p}, \mathrm{Q})$ & $0.437 \mathrm{~s}$ & N/A & 0.54 Gbits & N/A \\
\hline Total encounter & & & & 4.10 Gbits & 3.60 Gbits \\
\hline
\end{tabular}

In addition to the abbreviations described in Table 5, $\mathrm{M}$ indicates a specific mass/charge interval

Once these plasma data products have been produced, higher order data products, including lists of heliospheric current sheet crossings, interplanetary shocks and their properties, and flux ropes will be generated and updated on a per encounter basis. 
Table 7 SPC Science Data

\begin{tabular}{llllll}
\hline Product & Description & Survey cadence & Archive cadence* & Survey volume & Archive* $^{*}$ \\
\hline Proton/Alpha Full Sweep & $128 \mathrm{Ex} 4 \mathrm{I}$ & $0.874 \mathrm{~s}$ & - & $0.356 \mathrm{Gbits}$ & \\
Proton Peak Tracking & $8 \mathrm{Ex} 4 \mathrm{I}$ & $0.874 \mathrm{~s}$ & - & $4.385 \mathrm{Gbits}$ & - \\
Proton Flux-Angle & $1 \mathrm{Ex} 4 \mathrm{I}$ & $0.874 \mathrm{~s}$ & - & $0.070 \mathrm{Gbits}$ & - \\
Electron Full Sweep & $128 \mathrm{Ex} 4 \mathrm{I}$ & $10 \mathrm{~s}$ & - & $0.022 \mathrm{Gbits}$ & - \\
Electron Flux-Angle & $1 \mathrm{Ex} 4 \mathrm{I}$ & $10 \mathrm{~s}$ & - & $0.035 \mathrm{Gbits}$ & - \\
Total encounter & & & & $4.868 \mathrm{Gbits}$ & - \\
\hline
\end{tabular}

* The SPC data will not be archived on the SWEM but routed to the spacecraft data recorder

Table 8 Summary of data products from SWEAP sensors

SPC

Proton velocity, density, and temperature [key parameters]

$0.874 \mathrm{~s}(0.1 \mathrm{~s})$

Detailed fitted proton and alpha particle solar wind parameters

$0.874 \mathrm{~s}(0.1 \mathrm{~s})$

[anisotropies, secondary beam]

Rapid solar wind flow angle and flux variation

$0.874(0.0068 \mathrm{~s})$

Electron heat flux and flow angles

$10 \mathrm{~s}(0.0068 \mathrm{~s})$

SPAN

Full resolution ion and electron 3D Velocity distribution Functions (VDF) 56 second (0.437 s)

Ion and electron moments

0.437 seconds

Ion and electron energy spectra

0.437 seconds

Mass/charge spectra

7 minutes

\subsection{Science Operations}

SPP runs on a bent-pipe operations scheme, in which the SPP Mission Operations Center (MOC) and the SPP spacecraft will act only as a pass through of the command plans and telemetry to and from the SWEAP Investigation Science Operations Center (SOC). The SWEAP SOC will plan orbital command plans, select full resolution data, data processing, distribution and archiving. The SOC will also be utilized during integration and test activities, mission simulations, and to commission the SWEAP Suite. This section briefly summarizes SWEAP activities during commissioning and normal operations. A more detailed discussion of the SWEAP SOC initial design and implementation has been published elsewhere (Korreck et al. 2014).

Commissioning SWEAP commissioning consists of two sets of activities, the SWEAP Suite commissioning activities and multi-instrument commissioning activities. The SWEAP initial commissioning activities include the turn on, initialization, and functional testing of the SWEM and each of the sensors.. This initial commissioning will occur sufficiently early in the first orbit to overlap with measurements from existing missions in the heliosphere.

The multi-system commissioning will consist of activities that require more than just the SWEAP suite. These activities will take place after the initial commissioning activities for the SWEAP sensors. The first of the multi-system commissioning activities is a spacecraft 


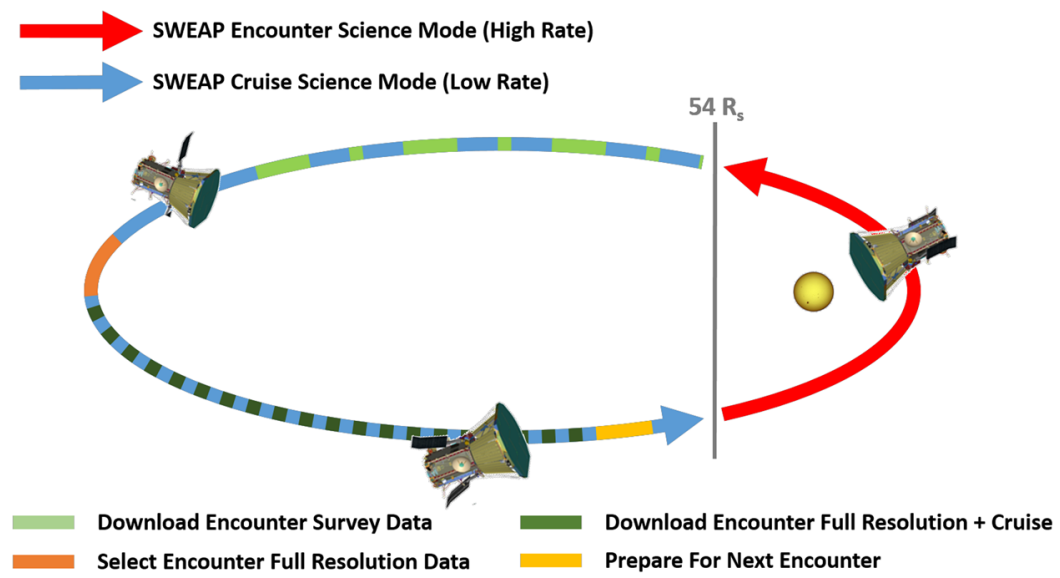

Fig. 29 Illustration of SWEAP activities based on notional phases of a SPP orbit. Primary science operations occur below 54 solar radii, although the instruments are sufficiently sensitive to detect the solar wind at greater distances if power is available

attitude slew of 45 degrees that will be performed in order to allow solar wind to flow into the SPAN-A ion instrument to perform an instrument checkout at thermal energies. A pause in the slew is performed at zero degrees for one minute so that the SPC can take measurements normal to the Sun. This slew will require the FIELDS MAG instrument to take magnetic field data. The second multi-system activity will be a test of the SWEAP-FIELDS direct data link, in which both instrument suites are powered on and a set of diagnostic tests are run.

Nominal Operations SWEAP conducts science observations in two overall modes: Encounter science mode close to the Sun with instruments operating at their highest sampling and data rates, and Cruise science mode with instruments operating in low power modes at greatly reduced sampling rates. SWEAP operations are summarized in the graphic in Fig. 29 and discussed below.

Below approximately 54 solar radii all SWEAP instruments are configured for encounter science mode, running at their highest cadences and executing a script of scheduled observation modes taking into

When SWEAP is in encounter science mode below 54 solar radii the suite produces two types of data products in the SWEM: full resolution and survey data. Survey data consists of the complete SPC dataset, along with a subsampling of the full resolution SPAN data and a series of moment calculations on the SPAN data. All full resolution SPAN data are archived onto flash memory within the SWEM. Following an encounter, all of the SPC data, and the SPAN survey data will be telemetered down to the ground and the science team will examine it for periods of interests based on scientific goals for the orbit. The SWEM will then be commanded to transfer the full-resolution SPAN data from those periods of interest to the SPP solid-state recorder. The full resolution data will then be telemetered during a downlink period later in the non-encounter phase of the orbit.

A baseline science observing plan is developed for each encounter for the SWEAP suite with inputs and in coordination with the SPP orbit planning. The free parameters in the SWEAP operation plans are the allowable energy ranges of the instruments and the relative fraction of time the instruments are configured to operate in the different scientific data 
Table 9 Data products by processing level

\begin{tabular}{|c|c|c|}
\hline Data level & Product title & Contents \\
\hline LO & Raw Telemetry files & $\begin{array}{l}\text { Raw CCSDS packets for SPC and SPANs } \\
\text { and auxiliary information such as } \\
\text { spacecraft ephemeris }\end{array}$ \\
\hline L1 & Instrument Count Rates (SPANs)/Currents (SPC) & Instrument responses not in physical units \\
\hline L2 & $\begin{array}{l}\text { Calibrated SPAN 3-D electron, proton, alpha, } \\
\text { and heavy ion distributions } \\
\text { Reduced SPC particle distribution functions } \\
\text { Onboard solar wind moments and energy spectra }\end{array}$ & $\begin{array}{l}\text { Data in physical units to be used for } \\
\text { scientific analysis }\end{array}$ \\
\hline L3 & $\begin{array}{l}\text { SPAN solar wind moments, energy spectra, } \\
\text { heavy ion distributions, and electron pitch angle } \\
\text { distributions } \\
\text { Solar wind moments from SPC reduced } \\
\text { distributions } \\
\text { Joint SPC-SPAN ion parameters }\end{array}$ & $\begin{array}{l}\text { Ground calculated parameters that } \\
\text { includes data from FIELDS. Data for } \\
\text { scientific analysis }\end{array}$ \\
\hline L4 & $\begin{array}{l}\text { Shock List } \\
\text { Event List } \\
\text { Power Spectra }\end{array}$ & Derived parameters from calibrated data \\
\hline
\end{tabular}

collection modes. SWEAP acquires science data over the approximately ten-day solar encounters when SPP is within 0.25 AU of the Sun. SWEM cycles SPC and SPAN through the scientific observing modes, creating onboard data products and saving full resolution SPAN data in an internal buffer. The SWEM sends all SPC data directly to the SPP solid state recorder (SSR). The SPAN data is processed in two ways. Once per second, a data packet goes to the SSR. All other full resolution data is placed in the SWEM solid state memory for selection by scientists. Data from SWEAP are processed and reviewed at the SWEAP Science Operations Center (SOC) as they become available from the MOC. Then the survey data will be analyzed by the science team to select which time periods of full resolution data from the SPAN instruments that is stored in the SWEM archive memory will be downlinked. A command plan will be uploaded to transfer those full resolution data from the SWEM solid state recorder to the spacecraft SSR to then be downlinked.

SWEAP will provide coarse sampling of the solar wind over the entire orbit including outside of 0.25 AU. While SWEAP operation over the entire SPP orbit is not required to meet SWEAP Level 1 science goals, there is value in producing radial overlap in observations by SPP and other spacecraft.

\subsection{Data Products}

The scientific data that is produced by the SWEAP Suite will be downloaded and processed by the SWEAP SOC. Table 9 describes the data products and their corresponding data level for the SWEAP suite. The raw telemetry in L0 and the slightly processed products in Level 1 will be utilized only by the SWEAP team as they are not useful for scientific analysis. Level 2, 3 and 4 data products, which include calibrate data in physical units, will be served to the scientific community for analysis.

The scientific data will be archived at SAO and UCB. The deep archive for the mission will be kept at NASA's Space Physics Data Facility (SPDF). The data will be openly avail- 
able to the rest of the SPP science teams and broader scientific community via the SWEAP website as well as through the Virtual Heliospheric Observatory.

\section{Summary and Conclusions}

Half a century into the space age, and more than half a century since a solar probe was first proposed, we are finally developing our first mission to the atmosphere of our Sun. Solar Probe Plus will allow us to answer fundamental questions about our closest star: Why is the corona millions of degrees hotter than the visible surface of the Sun? How does the corona produce the supersonic and variable solar wind? How are solar flares and coronal eruptions able to produce storms of energetic particle radiation? Successfully answering these questions would represent a major breakthrough in our understanding of the physics of energetic magnetized plasmas, allowing us to better understand and predict the evolution of the corona and solar wind and providing us with general insights into the physics of plasmas from the laboratory to exotic astrophysical environments.

The Solar Wind Electrons Alphas and Protons (SWEAP) Investigation will obtain the thermal coronal and solar wind plasma observations needed by the scientific community to address these compelling questions. The four SWEAP sensors provide complete measurements from several $\mathrm{eV}$ to tens of $\mathrm{keV}$ of the velocity distribution functions of electrons and ionized helium and hydrogen (alpha particles and protons) that constitute the bulk of the solar wind and coronal plasma, along with properties of other ions sorted by their mass/charge. Entering a new and harsh environment for the first time, the instruments face technical challenges unlike any other mission to date. As described in this paper, the SWEAP instruments presented at Preliminary Design Review can survive in these new environments and return the plasma observations needed for major progress in our understanding of the Sun. Engineering models of SWEAP instruments are currently being fabricated and tested in preparation for Critical Design Review in 2015.

Acknowledgements We acknowledge support from NASA under contract NNN06AA01C (Task NNN10AA08T). We are grateful for the creativity and dedication of the many people at SWEAP institutions who make this investigation possible. We also acknowledge early support for technology development by the Smithsonian Institution, NASA Marshall Space Flight Center and the CNRS PROMES research group in Odeillo-Font Romeu, France. We thank Jean-Yves Prado and CNES for providing financial support for our activities in France. We also thank NASA and the rest of the SPP team for their support on this project. J.C. Kasper personally thanks John Alexander Simpson and Alan Jay Lazarus for their contributions to Solar Probe and for exposing a student to this exciting mission of discovery.

Open Access This article is distributed under the terms of the Creative Commons Attribution 4.0 International License (http://creativecommons.org/licenses/by/4.0/), which permits unrestricted use, distribution, and reproduction in any medium, provided you give appropriate credit to the original author(s) and the source, provide a link to the Creative Commons license, and indicate if changes were made.

\section{References}

W.I. Axford, J.F. McKenzie, The origin of high speed solar wind streams, in Solar Wind Seven Colloquium, vol. 1 (1992)

S.D. Bale, J.C. Kasper, G.G. Howes, E. Quataert, C. Salem, D. Sundkvist, Magnetic fluctuation power near proton temperature anisotropy instability thresholds in the solar wind. Phys. Rev. Lett. 103, 211101 (2009)

Bale et al., Space Sci. Rev. (2015, this issue) 
B. Bavassano, E. Pietropaolo, R. Bruno, Alfvénic turbulence in the polar wind: a statistical study on cross helicity and residual energy variations. J. Geophys. Res. 105, 12697 (2000)

H. Bergner et al., Mechanical design of the Solar Probe Cup instrument on Solar Probe Plus, in Proc. SPIE 8862, Solar Physics and Space Weather Instrumentation V, vol. 88620L (2013). doi:10.1117/12.2022761

J.E. Borovsky, Flux tube texture of the solar wind: strands of the magnetic carpet at 1 AU? J. Geophys. Res. Space Phys. 113, A08110 (2008)

E.C. Bruner Jr., Dynamics of the solar transition zone. Astrophys. J. 226, 1146 (1978)

P.J. Cargill, J.A. Klimchuk, Nanoflare heating of the corona revisited. Astrophys. J. 605, 920 (2004)

C.W. Carlson, D.W. Curtis, G. Paschmann, W. Michael, An instrument for rapidly measuring plasma distribution functions with high resolution. Adv. Space Res. 2, 67-70 (1983)

C.W. Carlson, R.F. Pfaff, J.G. Watzin, The Fast Auroral SnapshoT (FAST) mission. Geophys. Res. Lett. 25, 2016 (1998)

A.W. Case et al., Designing a sun-pointing Faraday cup for solar probe plus, in AIP Conference Proceedings, vol. 1539 (2013), pp. 458-461

B.D.G. Chandran et al., Constraining low-frequency Alfvénic turbulence in the solar wind using densityfluctuation measurements. Astrophys. J. 707, 1675 (2009)

P. Cheimets et al., The design, development, and implementation of a solar environmental simulator (SES) for the SAO Faraday Cup on Solar Probe Plus, in Proc. SPIE 8862, Solar Physics and Space Weather Instrumentation $V$, vol. 88620M (2013). doi:10.1117/12.2024051

J. Cho, A. Lazarian, Particle acceleration by magnetohydrodynamic turbulence. Astrophys. J. 638, 811 (2006)

E.E. Chollet et al., Spatial offsets of interplanetary ion and electron source regions. Astrophys. J. 705, 1495 (2009)

J.W. Cirtain et al., Evidence for Alfvén waves in solar X-ray jets. Science 318, 1580 (2007)

S.R. Cranmer, Ion cyclotron wave dissipation in the solar corona: the summed effect of more than 2000 ion species. Astrophys. J. 532, 1197 (2000)

S.R. Cranmer, A.A. van Ballegooijen, R.J. Edgar, Self-consistent coronal heating and solar wind acceleration from anisotropic magnetohydrodynamic turbulence. Astrophys. J. Suppl. Ser. 171, 520 (2007)

N.U. Crooker, J.T. Gosling, S.W. Kahler, Reducing heliospheric magnetic flux from coronal mass ejections without disconnection. J. Geophys. Res. Space Phys. 107, 1028 (2002)

C.E. De Forest, S.P. Plunkett, M.D. Andrews, Observation of polar plumes at high solar altitudes. Astrophys. J. 546, 569 (2001)

B. de Pontieu et al., A tale of two spicules: the impact of spicules on the magnetic chromosphere. Publ. Astron. Soc. Jpn. 59, 655 (2007)

B. De Pontieu et al., Observing the roots of solar coronal heating-in the chromosphere. Astrophys. J. 701, L6 (2009)

P. Dmitruk et al., Coronal heating distribution due to low-frequency, wave-driven turbulence. Astrophys. J. $\mathbf{5 7 5}, 571$ (2002)

G. Einaudi et al., Formation of the slow solar wind in a coronal streamer. J. Geophys. Res. 104, 534 (1999)

L.A. Fisk, G. Gloeckler, The common spectrum for accelerated ions in the quiet-time solar wind. Astrophys. J. 640, L79 (2006)

L.A. Fisk, N.A. Schwadron, The behavior of the open magnetic field of the Sun. Astrophys. J. 560, 425 (2001)

L.A. Fisk, B. Kozlovsky, R. Ramaty, An interpretation of the observed oxygen and nitrogen enhancements in low-energy cosmic rays. Astrophys. J. 190, L35 (1974)

Fox et al., Space Sci. Rev. (2015, this issue)

Freeman et al., Technology development for the Solar Probe Plus Faraday Cup, in Proc. SPIE 8862, Solar Physics and Space Weather Instrumentation V, vol. 88620K (2013). doi:10.1117/12.2024983

V.L. Galinsky, V.I. Shevchenko, Nonlinear cyclotron resonant wave-particle interaction in a nonuniform magnetic field. Phys. Rev. Lett. 85, 90 (2000)

M.K. Georgoulis, M. Velli, G. Einaudi, Statistical properties of magnetic activity in the solar corona. Astrophys. J. 497, 957 (1998)

G. Gloeckler, Ion composition measurement techniques for space plasmas. Rev. Sci. Instrum. 61, 3613 (1990)

G. Gloeckler et al., Formation of power law tail with spectral index -5 inside and beyond the heliosphere. AIP Conf. Proc. 1039, 367 (2008)

J.T. Gosling et al., Bidirectional solar wind electron heat flux events. J. Geophys. Res. 92, 8519 (1987)

K.I. Gringauz et al., Results of observations of charged particles observed out to $\mathrm{R}=100000 \mathrm{~km}$, with the aid of charged-particle traps on soviet space rockets. Sov. Astron. 4, 680 (1960)

J.S. Halekas et al., The solar wind ion analyzer for MAVEN. Space Sci. Rev. (2013). doi:10.1007/s11214-0130029-z 
P. Hellinger et al., Proton thermal energetics in the solar wind: Helios reloaded. J. Geophys. Res. Space Phys. 118(4), 1351-1365 (2013)

J.V. Hollweg, Heating of the corona and solar wind by switch-on shocks. Astrophys. J. 254, 806 (1982)

J.V. Hollweg, The solar wind: our current understanding and how we got here. J. Astrophys. Astron. 29, 217 (2008)

J.V. Hollweg, P.A. Isenberg, Generation of the fast solar wind: a review with emphasis on the resonant cyclotron interaction. J. Geophys. Res. Space Phys. 107, 1147 (2002)

P.A. Isenberg, Heating of coronal holes and generation of the solar wind by ion-cyclotron resonance. Space Sci. Rev. 95, 119 (2001a)

P.A. Isenberg, The kinetic shell model of coronal heating and acceleration by ion cyclotron waves: 2 . Inward and outward propagating waves. J. Geophys. Res. 106, 29249 (2001b)

P.A. Isenberg, The kinetic shell model of coronal heating and acceleration by ion cyclotron waves: 3 . The proton halo and dispersive waves. J. Geophys. Res. Space Phys. 109, 3101 (2004)

J.C. Kasper et al., Wind/SWE observations of firehose constraint on solar wind proton temperature anisotropy. Geophys. Res. Lett. (2002). doi:10.1029/2002GL015128

J.C. Kasper et al., Solar wind temperature anisotropies. AIPC News 679, 541 (2003)

J.C. Kasper et al., Physics-based tests to identify the accuracy of solar wind ion measurements: a case study with the wind Faraday cups. J. Geophys. Res. A 111, A03105 (2006)

J.C. Kasper, A.J. Lazarus, S.P. Gary, Hot solar-wind helium: direct evidence for local heating by Alfvéncyclotron dissipation. Phys. Rev. Lett. 101, 26 (2008)

J.C. Kasper et al., Evolution of the relationships between helium abundance, minor ion charge state, and solar wind speed over the solar cycle. Astrophys. J. 2012, 745 (2012)

J.C. Kasper et al., Sensitive test for ion-cyclotron resonant heating in the solar wind. Phys. Rev. Lett. 2013, 110 (2013)

K.E. Korreck et al., Solar Wind Electrons Alphas and Protons (SWEAP) science operations center initial design and implementation. Proceedings of the SPIE, vol. 9149 (2014)

J.A. Le Roux, G.P. Zank, W.H. Matthaeus, Pickup ion acceleration by turbulent field-aligned electric fields in the slow low-latitude solar wind. J. Geophys. Res. Space Phys. 107, 1138 (2002)

R.J. Leamon et al., Observational constraints on the dynamics of the interplanetary magnetic field dissipation range. J. Geophys. Res. 103, 4775 (1998)

G. Li, Identifying current-sheet-like structures in the solar wind. Astrophys. J. Lett. 672, 65 (2008)

R.P. Lin et al., A three-dimensional plasma and energetic particle investigation for the wind spacecraft. Space Sci. Rev. 71, 153 (1995)

D.W. Longcope, T.C.B. McLeish, G.H. Fisher, A viscoelastic theory of turbulent fluid permeated with fibril magnetic fields. Astrophys. J. 599, 674 (2003)

E. Marsch, C.-Y. Tu, Heating and acceleration of coronal ions interacting with plasma waves through cyclotron and Landau resonance. J. Geophys. Res. 106, 227 (2001)

Maruca et al., What are the relative roles of heating and cooling in generating solar wind temperature anisotropies?. Phys. Rev. Lett. 107, 201101 (2011)

B.A. Maruca, S.D. Bale, L. Sorriso-Valvo, J.C. Kasper, M.L. Stevens, Collisional thermalization of hydrogen and helium in solar-wind plasma. Phys. Rev. Lett. 111, 24 (2013)

W.H. Matthaeus, M.L. Goldstein, D.A. Roberts, Evidence for the presence of quasi-two-dimensional nearly incompressible fluctuations in the solar wind. J. Geophys. Res. 95, 20673 (1990)

W.H. Matthaeus et al., Coronal heating by magnetohydrodynamic turbulence driven by reflected lowfrequency waves. Astrophys. J. Lett. 523, 93 (1999)

D.J. McComas, M. Velli, W.S. Lewis, L.W. Acton, M. Balat-Pichelin, V. Bothmer, R.B. Dirling Jr., W.C. Feldman, G. Gloeckler, S.R. Habbal, D.M. Hassler, I. Mann, W.H. Matthaeus, R.L. McNutt Jr., R.A. Mewaldt, N. Murphy, L. Ofman, E.C. Sittler Jr., C.W. Smith, T.H. Zurbuchen, Understanding coronal heating and solar wind acceleration: Case for in situ near-sun measurements. Rev. Geophys. 45, RG1004 (2007). doi:10.1029/2006RG000195

McComas et al., Integrated Science Investigation of the Sun (ISIS): design of the energetic particle investigation. Space Sci. Rev. (2014). doi:10.1007/s11214-014-0059-1

J.P. McFadden, C.W. Carlson, Computer Simulation in Designing Electrostatic Optics for Space Plasma Experiments. Geophysical Monograph Series, vol. 102 (1998)

J.P. McFadden et al., The THEMIS ESA plasma instrument and in-flight calibration. Space Sci. Rev. 141, 302 (2008)

McFadden et al., Space Sci. Rev. (2015, submitted)

McIntosh et al., Solar cycle variations in the elemental abundance of helium and fractionation of iron in the fast solar wind: indicators of an evolving energetic release of mass from the lower solar atmosphere. Astrophys. J. Lett. 740, L23 (2011)

J.A. Miller, Particle acceleration in impulsive solar flares. Space Sci. Rev. 86, 79 (1998) 
D.L. Mitchell et al., Probing Mars' crustal magnetic field and ionosphere with the MGS electron reflectometer. J. Geophys. Res. 106, 23428 (2001)

T. Mulligan, C.T. Russell, J.G. Luhmann, Solar cycle evolution of the structure of magnetic clouds in the inner heliosphere. Geophys. Res. Lett. 25, 2959 (1998)

L. Ofman, T. Wang, Propagating Alfven waves in coronal loops observed by Hinode, in AGU Fall Meeting, vol. SH52C-02 (2007)

K.W. Ogilvie et al., High-velocity tails on the velocity distribution of solar wind ions. J. Geophys. Res. 98, 3611 (1993)

T.J. Okamoto et al., Coronal transverse magnetohydrodynamic waves in a solar prominence. Science 318, 1577-1580 (2007)

E.N. Parker, Cosmic Magnetic Field (Clarendon, Oxford, 1979)

E.N. Parker, Stimulated dissipation of magnetic discontinuities and the origin of solar flares. Sol. Phys. 111, 297 (1987)

E.N. Parker, Nanflares and the solar X-ray corona. Astrophys. J. 330, 474 (1988)

V. Pierrard, H. Lamy, The effects of the velocity filtration mechanism on the minor ions of the corona. Sol. Phys. 216, 47 (2003)

W.G. Pilipp et al., Characteristics of electron velocity distribution functions in the solar wind derived from the HELIOS plasma experiment. J. Geophys. Res. 92, 1075 (1987)

E.R. Priest, J.F. Heyvaerts, A.M. Title, A flux-tube tectonics model for solar coronal heating driven by the magnetic carpet. Astrophys. J. 576, 533 (2002)

V.S. Ptuskin, Cosmic-ray acceleration by long-wave turbulence. Sov. Astron. Lett. 14, 255 (1988)

J.M. Raines et al., MESSENGER observations of the plasma environment near Mercury. Planetary and Space Science (2011). doi:10.1016/j.pss.2011.02.004

N.-E. Raouafi et al., Evidence for polar jets as precursors of polar plume formation. Astrophys. J. 682, L140 (2008)

J.D. Richardson, The solar wind in the foreshock upstream of the termination shock, in AGUFMSH11 (2007)

D.A. Roberts et al., Origin and evolution of fluctuations in the solar wind-HELIOS observations and HeliosVoyager comparisons. J. Geophys. Res. 92, 12023 (1987)

J. Sauvaud et al., Dynamic response of the far tail of the Earth magnetosphere to IMF changes and magnetospheric activity: a STEREO view, in AGUFMSM23 (2007)

C.J. Schrijver et al., Sustaining the quiet photospheric network: the balance of flux emergence, fragmentation, merging, and cancellation. Astrophys. J. 487, 436 (1997)

N.A. Schwadron et al., Coronal Electron temperature from the solar wind scaling law throughout the space age. Astrophys. J. 739, 9 (2011)

N.A. Schwadron et al., Coronal electron temperature in the protracted solar minimum, the cycle 24 mini maximum, and over centuries. J. Geophys. Res. 119(3), 1486-1492 (2014)

J.D. Scudder, Ion and electron suprathermal tail strengths in the transition region: support for the velocity filtration model of the corona. Astrophys. J. 427, 446 (1994)

D. Shaikh et al., Theory and simulations of principle of minimum dissipation rate. Phys. Plasmas 15, 12306 (2008)

N.R. Sheeley et al., Measurements of flow speeds in the corona between 2 and $30 \mathrm{R}_{\text {Sun. Astrophys. J. 484, }}$ 472 (1997)

N.R. Sheeley et al., The structure of streamer blobs. Astrophys. J. 694, 1471 (2009)

P.A. Sturrock, Chromospheric magnetic reconnection and its possible relationship to coronal heating. Astrophys. J. 521, 451 (1999)

Š. Štverák et al., Radial evolution of nonthermal electron populations in the low-latitude solar wind: Helios, Cluster, and Ulysses Observations. J. Geophys. Res. (2009). doi:10.1029/2008JA013883

S. Tomczyk et al., Alfvén waves in the solar corona. Science 317, 1192 (2007)

P. Ulmschneider, Propagation of magneto-acoustic waves along solar flux tubes, in Theoretical Problems in High Resolution Solar Physics (1985)

P. Ulmschneider, R.F. Stein, Heating of stellar chromospheres when magnetic fields are present. Astron. Astrophys. 106, 13 (1982)

V.M. Vasyliunas, Thermal and suprathermal plasmas in the magnetosphere. Comments Astrophys. Space Phys. 3, 48 (1971)

N.M. Viall, H.E. Spence, J. Kasper, Are periodic solar wind number density structures formed in the solar corona? Geophys. Res. Lett. 36, 23102 (2009)

Vourlidas et al., The Wide-Field Imager for Solar Probe Plus (WISPR). Space Sci. Rev. (2015). doi:10.1007/s11214-014-0114-y

Y.-M. Wang, Flux-tube divergence, coronal heating, and the solar wind. Astrophys. J. 410, L126 (1993)

Y.-M. Wang, N.R. Sheeley Jr., Footpoint switching and the evolution of coronal holes. Astrophys. J. 612, 1205 (2004) 
G.M. Webb et al., Diffusive-compression acceleration and turbulent diffusion of cosmic rays in quasi-periodic and turbulent flows. Astrophys. J. 595, 195 (2003)

G.P. Zank, W.H. Matthaeus, C.W. Smith, Evolution of turbulent magnetic fluctuation power with heliospheric distance. J. Geophys. Res. 101, 17093 (1996)

Y. Zhou, W.H. Matthaeus, Transport and turbulence modeling of solar wind fluctuations. J. Geophys. Res. 95, 10291 (1990)

T.H. Zurbuchen et al., The solar wind composition throughout the solar cycle: a continuum of dynamic states. Geophys. Res. Lett. 29, 66 (2002) 